Factorial Designs, Model Selection, and (Incorrect) Inference in Randomized Experiments

Karthik Muralidharan, Mauricio Romero, and Kaspar Wüthrich

NBER Working Paper No. 26562

December 2019, Revised in September 2020

JEL No. C12,C18,C90,C93

\begin{abstract}
Factorial designs are widely used for studying multiple treatments in one experiment. While ttests based on the "long" model (including main and interaction effects) provide valid inferences against "business-as-usual" counterfactuals, "short" model t-tests (that ignore interactions) yield higher power if the interactions are zero, but incorrect inferences otherwise. Out of 27 factorial experiments published in top-5 journals in 2007-2017, 19 use the short model. We reanalyze these experiments, and show that over half of their published results lose significance when interactions are included. We show that testing the interactions using the long model and presenting the short model if the interactions are not significantly different from zero leads to incorrect inference due to the implied data-dependent model selection. Based on recent econometric advances, we show that local power improvements over the long model are possible. However, if the main effects are of primary interest, leaving the interaction cells empty yields valid inferences and global power improvements. In addition, the sample size needed to detect interactions is substantially larger than that required to detect main effects, resulting in most experiments being under-powered to detect interactions. Thus, using factorial designs to explore whether interactions are meaningful can be problematic because interaction estimates are likely to considerably overestimate the magnitude of the true effect conditional on being significant.
\end{abstract}

Karthik Muralidharan

Department of Economics, 0508

University of California, San Diego

9500 Gilman Drive

La Jolla, CA 92093-0508

and NBER

kamurali@ucsd.edu

Mauricio Romero

Centro de Investigacion Economica

ITAM

Mexico

mtromero@itam.mx
Kaspar Wüthrich

University of California at San Diego

kwuthrich@ucsd.edu

A online appendix is available at http://www.nber.org/data-appendix/w26555 


\title{
Factorial designs, model selection, and (incorrect) inference in randomized experiments*
}

\author{
Karthik Muralidharan ${ }^{\dagger}$
}

\author{
Mauricio Romero $\ddagger$
}

\author{
Kaspar Wüthrich ${ }^{\S}$
}

September 4, 2020

\begin{abstract}
Factorial designs are widely used for studying multiple treatments in one experiment. While $t$-tests based on the "long" model (including main and interaction effects) provide valid inferences against "business-as-usual" counterfactuals, "short" model $t$-tests (that ignore interactions) yield higher power if the interactions are zero, but incorrect inferences otherwise. Out of 27 factorial experiments published in top-5 journals in 2007-2017, 19 use the short model. We reanalyze these experiments, and show that over half of their published results lose significance when interactions are included. We show that testing the interactions using the long model and presenting the short model if the interactions are not significantly different from zero leads to incorrect inference due to the implied data-dependent model selection. Based on recent econometric advances, we show that local power improvements over the long model are possible. However, if the main effects are of primary interest, leaving the interaction cells empty yields valid inferences and global power improvements. In addition, the sample size needed to detect interactions is substantially larger than that required to detect main effects, resulting in most experiments being under-powered to detect interactions. Thus, using factorial designs to explore whether interactions are meaningful can be problematic because interaction estimates are likely to considerably overestimate the magnitude of the true effect conditional on being significant.
\end{abstract}

Keywords: randomized controlled trials; cross-cut designs; power in field experiments; datadependent model selection; interaction effects; type-M errors

JEL Codes: C12, C18, C21, C90, C93

*We are grateful to Isaiah Andrews, Tim Armstrong, Prashant Bharadwaj, Arun Chandrasekhar, Clement de Chaisemartin, Gordon Dahl, Stefano DellaVigna, Esther Duflo, Graham Elliott, Markus Goldstein, Macartan Humphreys, Hiroaki Kaido, Lawrence Katz, Michal Kolesar, Soonwoo Kwon, Adam McCloskey, Craig McIntosh, Rachael Meager, Paul Niehaus, Ben Olken, Gautam Rao, Andres Santos, Jesse Shapiro, Diego Vera-Cossio, and several seminar participants for comments and suggestions. We are also grateful to the authors of the papers we reanalyze for answering our questions and fact-checking that their papers are characterized correctly. Sameem Siddiqui provided excellent research assistance. All errors are our own. Financial support from the Asociación Mexicana de Cultura, A.C. is gratefully acknowledged by Romero.

${ }^{\dagger}$ Department of Economics, UC San Diego; NBER; J-PAL; E-mail: kamurali@ucsd.edu

‡Centro de Investigación Económica, ITAM, Mexico City, Mexico; J-PAL; E-mail: mtromero@itam.mx

$\S$ Department of Economics, UC San Diego; E-mail: kwuthrich@ucsd.edu 


\section{Introduction}

Cross-cutting or factorial designs are widely used in field experiments. For example, 27 out of 124 field experiments published in top-5 economics journals during 20072017 use cross-cutting designs. One rationale for these designs is that the power for detecting main treatment effects against a "business-as-usual" counterfactual (where no one is exposed to any of the treatments analyzed in the experiment) is higher if the interactions between treatments are zero. This can make factorial designs a cost-effective way of studying multiple treatments. ${ }^{1}$ A second rationale is to "explore" if there are meaningful interactions across treatments. This paper is motivated by the observation that both of these rationales can be problematic in practice.

To fix ideas, consider a setup with two randomly-assigned binary treatments. The researcher can estimate either a fully-saturated "long" model (with dummies for both treatments and for their interaction) or a "short" model (only including dummies for both treatments). The long model yields consistent estimators for the average treatment effect of both treatments, as well as the interaction, and is always correct for inference regardless of the true value of the interaction. However, if the true value of the interaction effect is zero, the short model yields consistent estimators and also has greater power for conducting inference on the main treatment effects.

The power gains from the short model, however, come at the cost of an increased likelihood of incorrect inference relative to a business-as-usual counterfactual if the interaction effect is not zero. Out of 27 field experiments published in top-5 economics journals during 2007-2017 using cross-cutting designs, 19 do not include all interaction terms in the main specifications. We reanalyzed the data from these papers by also including the interaction terms. ${ }^{2}$ Doing so has non-trivial implications for inference on the main treatment effects. The median absolute value of the change in the point estimates is $96 \%$, about $26 \%$ of estimates change sign, and $53 \%$ ( 29 out of 55 ) of estimates reported to be significant at the $5 \%$ level are no longer so after including interactions. Even if we reanalyze only "policy" experiments, $32 \%$ of the estimates (6 out of 19) are no longer significant after including all interactions. ${ }^{3}$

\footnotetext{
${ }^{1}$ As Kremer (2003) puts it: "Conducting a series of evaluations in the same area allows substantial cost savings...Since data collection is the most costly element of these evaluations, cross-cutting the sample reduces costs dramatically...This tactic can be problematic, however, if there are significant interactions between programs".

${ }^{2}$ The full list of 27 papers is in Table A.1. We reanalyzed 15 out of the 19 that do not include all interactions in the main specification. The other four papers did not have publicly-accessible data.

${ }^{3}$ We define a policy experiment as one which studies a program or intervention that could be scaled up; as opposed to a conceptual experiment, which aims to test for the existence of facts or concepts such as discrimination (e.g., resume audit experiments).
} 
In practice, researchers often try to address the issue of interactions by first estimating the long model and testing if the interaction is significant, and then focusing on the short model if they do not reject that the interaction is zero. However, the distributions of the estimators obtained from this data-dependent model selection procedure are complicated and highly non-normal, making the usual $t$-statistics misleading (Leeb \& Pötscher, 2005 , 2006, 2008). Further, cross-cutting experiments are rarely adequately powered to detect meaningful interactions (see Figure 2). Thus, this two-step procedure will almost always fail to reject that the interaction term is zero, even when it is different from zero. As a result, the rate of incorrect inference using this two-step model-selection procedure will continue to be nearly as high as that from just running the short model.

The lack of power to detect interactions combined with a focus on statistical significance also makes it challenging to use factorial designs to "explore" whether interactions are meaningful. We show in Section 7 that the variance of the interaction estimator is always larger than that of the main effects estimators, which makes the sample size requirements for detecting interactions much more onerous. For example, one would need an 8 times larger sample to detect an interaction than to detect a main effect when the interaction is half the size of the main effect. This leads to most studies being underpowered to detect interactions. Thus, significant interactions are likely to considerably overestimate the magnitude of the true effect, and hence be misleading. This problem has been referred to by Gelman \& Carlin (2014) as exaggeration ratio or Type-M error.

Textbook treatments of factorial designs (Cochran \& Cox, 1957; Gerber \& Green, 2012) and guides to practice (Kremer, 2003; Duflo et al., 2007) are careful to clarify that treatment effects using the short model should be interpreted as either (a) being conditional on the distribution of the other treatment arms in the experiment, or (b) as a composite treatment effect that includes a weighted-average of the interactions with other treatments. However, as we argue in Section 2.3, this weighted average is a somewhat arbitrary construct, can be difficult to interpret in high-dimensional factorial designs, and is typically neither of primary academic interest nor policy-relevant. Consistent with this view, none of the 19 experimental papers in Table A.1 that focus on the short model motivate their experiment as being about estimating a weighted-average treatment effect.

The status quo of focusing on the short-model is problematic for at least three reasons. First, ignoring interactions affects internal validity against a "business-as-usual" counterfactual. If the interventions studied are new, the other programs may not even exist in the study population. Even if they do, there is no reason to believe that the distributions in the population mirror those in the experiment. Thus, to the extent that estimation and inference of treatment effects depend on what other interventions are being studied in 
the same experiment, ignoring interactions is a threat to internal validity.

Second, "absence of evidence" of significant interactions may be getting erroneously interpreted as "evidence of absence". The view that interactions are second-order (as implied when papers only present the short model) may have been influenced in part by the lack of evidence of meaningful interactions in most experiments to date. However, as we show in Section 7, this is at least partly because few experiments are adequately powered to detect meaningful interactions. There is now both experimental (Duflo et al., 2015a; Mbiti et al., 2019) and non-experimental (Kerwin \& Thornton, 2017; Gilligan et al., 2018) evidence that interactions matter. Indeed, a long tradition in development economics has highlighted the importance of complementarities across programs in alleviating poverty traps (Ray, 1998; Banerjee \& Duflo, 2005), which suggests that assuming away interactions in empirical work may be a mistake.

Third, there is well-documented publication bias towards significant findings (e.g., Abadie, 2020; I. Andrews \& Kasy, 2018; Christensen \& Miguel, 2018; Franco et al., 2014). This can also affect evidence aggregation because meta-analyses and evidence reviews often only include published studies. Thus, the sensitivity of the significance of main effect estimates to the inclusion/exclusion of interaction terms (which we document in this paper), is likely to have non-trivial implications for how evidence is published, summarized, and translated into policy.

Having documented the limitations of the short model, we consider if it is possible to improve power relative to the long model while maintaining size control for relevant values of the interactions. Since the two-sided $t$-test based on the long model is the uniformly most powerful unbiased test (e.g., van der Vaart, 1998), any procedure more powerful than the $t$-test for some values of the interactions must underperform somewhere else. Keeping this constraint in mind, we explore four possible econometric approaches.

The first approach, based on Elliott et al. (2015), is a nearly optimal test that targets power towards an a priori likely value of the interaction (such as a value of zero), while controlling size for all values of the interaction. This approach comes close to achieving the maximal possible power gains near the likely values of the interaction, while exhibiting lower power farther away from this value. The nearly optimal test can be useful in $2 \times 2$ factorial designs, but becomes computationally prohibitive in more complicated factorial designs. ${ }^{4}$ Our second approach, based on Armstrong et al. (2019), is to construct confidence intervals for the main effects under prior knowledge on the magnitude of the interactions. Incorporating prior knowledge is computationally feasible even in compli-

\footnotetext{
${ }^{4}$ Our code to implement this procedure for $2 \times 2$ factorial designs is available at https://mtromero .shinyapps.io/elliott/
} 
cated factorial designs but requires prior knowledge on potentially many interactions to yield notable power improvements. When the prior knowledge is correct, this approach controls size and yields power gains relative to the $t$-test based on the long model. However, it suffers from size distortions if the prior knowledge is incorrect. In the appendix, we explore two additional econometric approaches based on work by Imbens \& Manski (2004), Stoye (2009), and McCloskey (2017). ${ }^{5}$

Based on the analysis above, we recommend that all completed factorial experiments report results from the long regression model. $t$-tests based on the long model are easy to compute even in complicated factorial designs and have appealing optimality properties. Further, the justification for the short model should not be that the interactions were not significant in the long model (because of the model selection issue discussed above). Rather, if researchers would like to focus on results from the short model, they should clearly indicate that treatment effects should be interpreted as a composite treatment effect that includes a weighted-average of interactions with other treatments (and specify the estimand of interest in a pre-analysis plan). This will ensure transparency in the interpretation of the main results and enable readers to assess the extent to which other treatments may be typical background factors that can be ignored.

For the design of new policy experiments, if the primary parameters of interest are the main effects, a natural alternative is to leave the "interaction cells" empty and increase the number of units assigned to the main treatment(s) or the control group. Our simulations show that this design-based approach yields more power gains than the econometric methods discussed above for most of the relevant values of the interaction.

Reviewing classic texts on experimental design, we identify four cases where factorial designs and analyses of the short model may be appropriate. The first is where the goal is to explore several treatments efficiently to identify promising interventions for further testing. ${ }^{6}$ However, most policy experiments are run only once, which makes factorial designs and short model estimates less desirable in these settings.

The second is when the goal of the experiment is not hypothesis testing but to minimize mean squared error (MSE) criteria (or other loss functions), which involve a biasvariance trade-off in estimating the main effects (e.g., Blair et al., 2019). ${ }^{7}$ However, a key rationale for experimental evaluations of policies and programs is to generate unbiased

\footnotetext{
${ }^{5}$ Our simulations show that these are unlikely to yield meaningful power improvements relative to the first two approaches and the long model $t$-test.

${ }^{6}$ For example, Cochran \& Cox (1957, p.152) recommend factorial designs for "exploratory work where the object is to determine quickly the effects of a number of factors over a specified range".

${ }^{7}$ Two classes of experiments that satisfy the first and second criteria are: (a) agricultural experiments, and (b) online A/B testing (e.g., Kohavi et al., 2020). Both feature sequential testing and aim to optimize decision making over several factors as opposed to testing if individual factors are "significant".
} 
estimates of program impact, which makes the bias in the short model unattractive.

The third is to improve external validity. Cochran \& Cox (1957, p.152) recommend bringing in subsidiary factors in factorial designs to test main effects over a wide range of conditions; also see Fisher (1992). Thus, factorial designs and analyses of the short model may be fine when one dimension of the experiment is studying reasonable variants of the main treatment, but less so when both treatments are of primary interest. ${ }^{8}$

The fourth is the case of conceptual (as opposed to policy) experiments, such as resume audit studies, where many of the characteristics that are randomized (such as age, education, race, and gender) do exist in the population. However, when feasible, we recommend having the treatment share of various characteristics being studied be the same as their population proportion. Doing so will make the short-model coefficient more likely to approximate a population relevant parameter of interest.

Our most important contribution is to the literature on the design of field experiments. Athey \& Imbens (2017), Bruhn \& McKenzie (2009), and List et al. (2011) provide guidance on the design of field experiments, but do not discuss when and when not to implement factorial designs. Duflo et al. (2007) implicitly endorse the use of factorial designs by noting that they "[have] proved very important in allowing for the recent wave of randomized evaluations in development economics".

Our reanalysis of existing experiments as well as simulations suggest that there is no free lunch. The perceived gains in power and cost-effectiveness from running experiments with factorial designs come at the cost of not controlling size and an increased rate of false positives relative to a business-as-usual counterfactual. Alternatively, they come at the cost of a more complicated interpretation of the main results as including a weighted-average of interactions with other treatments that may not represent a typical counterfactual. Further, using under-powered factorial designs to explore whether interactions are significant comes at the risk of overestimating their true effect.

We also contribute to the literature that aims to improve the analysis of field experiments (e.g., List et al., 2016; Young, 2018). Our paper follows in this tradition by documenting a problem with the status quo, quantifying its importance, and identifying the most relevant recent advances in theoretical econometrics that can mitigate the problem. Specifically, we show that the econometric analysis of nonstandard inference problems can be brought to bear to improve inference in factorial designs which are ubiquitous in field experiments.

\footnotetext{
${ }^{8}$ For example, in Alatas et al. (2012), the primary treatment effect of interest is the impact of communitybased targeting, but they also randomize different aspects of how to run the community meeting (which are reasonable variants of the main treatment but not of primary interest).
} 


\section{Theoretical analysis of factorial designs}

In this section, we discuss identification, estimation, and inference in experiments with factorial (or "cross-cut") designs. For simplicity, we focus on factorial designs with two treatments, $T_{1}$ and $T_{2}$ (commonly known as " $2 \times 2$ designs"), where a researcher randomly assigns some subjects to receive treatment $T_{1}$, some subjects to receive treatment $T_{2}$, and some subjects to receive both treatments (see Table 1). It is straightforward to extend the analysis to cross-cut designs with more than two treatments; we do so in Section 6.

Table 1: $2 \times 2$ factorial design

\begin{tabular}{|c|c|c|c|}
\hline & \multicolumn{2}{|c|}{$T_{1}$} \\
\hline & & No & Yes \\
\hline \multirow{2}{*}{$T_{2}$} & No & $N_{1}$ & $N_{2}$ \\
\hline & Yes & $N_{3}$ & $N_{4}$ \\
\hline
\end{tabular}

Note: $N_{j}$ is the number of individuals randomly assigned to cell $j$.

\subsection{Potential outcomes and treatment effects}

We formalize the problem using the potential outcomes framework of Rubin (1974). Our goal is to identify and estimate the causal effect of the two treatments, $T_{1}$ and $T_{2}$, on an outcome of interest, $Y$. Potential outcomes $\left\{Y_{t_{1}, t_{2}}\right\}$ are indexed by both treatments $T_{1}=t_{1}$ and $T_{2}=t_{2}$, and are related to the observed outcome as

$$
Y=Y_{0,0} \cdot \mathbf{1}_{\left\{T_{1}=0, T_{2}=0\right\}}+Y_{1,0} \cdot \mathbf{1}_{\left\{T_{1}=1, T_{2}=0\right\}}+Y_{0,1} \cdot \mathbf{1}_{\left\{T_{1}=0, T_{2}=1\right\}}+Y_{1,1} \cdot \mathbf{1}_{\left\{T_{1}=1, T_{2}=1\right\}},
$$

where $\mathbf{1}_{\{A\}}$ is an indicator function which is equal to one if the event $A$ is true and zero otherwise. There are different types of average treatment effects (ATEs):

$$
\begin{array}{ll}
E\left(Y_{1,0}-Y_{0,0}\right): & \text { ATE of } T_{1} \text { relative to a counterfactual where } T_{2}=0 \\
E\left(Y_{0,1}-Y_{0,0}\right): & \text { ATE of } T_{2} \text { relative to a counterfactual where } T_{1}=0 \\
E\left(Y_{1,1}-Y_{0,1}\right): & \text { ATE of } T_{1} \text { relative to a counterfactual where } T_{2}=1 \\
E\left(Y_{1,1}-Y_{1,0}\right): & \text { ATE of } T_{2} \text { relative to a counterfactual where } T_{1}=1 \\
E\left(Y_{1,1}-Y_{0,0}\right): & \text { ATE of } T_{1} \text { and } T_{2} \text { combined }
\end{array}
$$

We refer to $E\left(Y_{1,0}-Y_{0,0}\right)$ and $E\left(Y_{0,1}-Y_{0,0}\right)$ as the main treatment effects of $T_{1}$ and $T_{2}$ relative to a business-as-usual counterfactual, where no one is exposed to either treatment 
analyzed in the experiment. The interaction effect - the difference between the effect of jointly providing both treatments and the sum of the main effects - is

$$
E\left(Y_{1,1}-Y_{0,0}\right)-\left[E\left(Y_{1,0}-Y_{0,0}\right)+E\left(Y_{0,1}-Y_{0,0}\right)\right]=E\left(Y_{1,1}-Y_{0,1}-Y_{1,0}+Y_{0,0}\right)
$$

We assume that both treatments are randomly assigned and independent of each other such that the different ATEs are identified as

$$
\begin{aligned}
& E\left(Y_{1,0}-Y_{0,0}\right)=E\left(Y \mid T_{1}=1, T_{2}=0\right)-E\left(Y \mid T_{1}=0, T_{2}=0\right), \\
& E\left(Y_{0,1}-Y_{0,0}\right)=E\left(Y \mid T_{1}=0, T_{2}=1\right)-E\left(Y \mid T_{1}=0, T_{2}=0\right), \\
& E\left(Y_{1,1}-Y_{0,1}\right)=E\left(Y \mid T_{1}=1, T_{2}=1\right)-E\left(Y \mid T_{1}=0, T_{2}=1\right), \\
& E\left(Y_{1,1}-Y_{1,0}\right)=E\left(Y \mid T_{1}=1, T_{2}=1\right)-E\left(Y \mid T_{1}=1, T_{2}=0\right), \\
& E\left(Y_{1,1}-Y_{0,0}\right)=E\left(Y \mid T_{1}=1, T_{2}=1\right)-E\left(Y \mid T_{1}=0, T_{2}=0\right),
\end{aligned}
$$

and the interaction effect is identified via Equation (2).

\subsection{Long and short regression models}

Researchers analyzing experiments based on cross-cut designs typically consider one of the following two population regression models:

$$
\begin{aligned}
& Y=\beta_{0}+\beta_{1} T_{1}+\beta_{2} T_{2}+\beta_{12} T_{1} T_{2}+\varepsilon, \quad \text { (long model) } \\
& Y=\beta_{0}^{s}+\beta_{1}^{s} T_{1}+\beta_{2}^{s} T_{2}+\varepsilon^{s} \quad \text { (short model) }
\end{aligned}
$$

The fully saturated "long" model (3) includes both treatment indicators as well as their interaction. By contrast, the "short" model (4) only includes the two treatment indicators, but ignores the interaction term.

We now relate the population regression coefficients in these models to the causal effects defined in Section 2.1; see Appendix A.2 for detailed derivations. ${ }^{9}$ The coefficients in the long regression model correspond to the main effects of $T_{1}$ and $T_{2}$ against a

\footnotetext{
${ }^{9}$ The population regression coefficient $\beta$ in the model $Y=X^{\prime} \beta+\varepsilon$ is the solution to the population least squares problem and is given by $\beta=E\left(X X^{\prime}\right)^{-1} E(X Y)$.
} 
business-as-usual counterfactual, and the interaction effect:

$$
\begin{aligned}
\beta_{1} & =E\left(Y_{1,0}-Y_{0,0}\right) \\
\beta_{2} & =E\left(Y_{0,1}-Y_{0,0}\right) \\
\beta_{12} & =E\left(Y_{1,1}-Y_{0,1}-Y_{1,0}+Y_{0,0}\right) .
\end{aligned}
$$

By contrast, the regression coefficients in the short model are

$$
\begin{aligned}
\beta_{1}^{s} & =E\left(Y_{1,1}-Y_{0,1}\right) P\left(T_{2}=1\right)+E\left(Y_{1,0}-Y_{0,0}\right) P\left(T_{2}=0\right) \\
& =E\left(Y_{1,0}-Y_{0,0}\right)+E\left(Y_{1,1}-Y_{0,1}-Y_{1,0}+Y_{0,0}\right) P\left(T_{2}=1\right) \\
& =\beta_{1}+\beta_{12} P\left(T_{2}=1\right)
\end{aligned}
$$

and

$$
\begin{aligned}
\beta_{2}^{s} & =E\left(Y_{1,1}-Y_{1,0}\right) P\left(T_{1}=1\right)+E\left(Y_{0,1}-Y_{0,0}\right) P\left(T_{1}=0\right) \\
& =E\left(Y_{0,1}-Y_{0,0}\right)+E\left(Y_{1,1}-Y_{0,1}-Y_{1,0}+Y_{0,0}\right) P\left(T_{1}=1\right) \\
& =\beta_{2}+\beta_{12} P\left(T_{1}=1\right)
\end{aligned}
$$

Equation (8) shows that $\beta_{1}^{s}$ yields a weighted average of the ATE of $T_{1}$ relative to a counterfactual where $T_{2}=1$ and the ATE of $T_{1}$ relative to a business-as-usual counterfactual where $T_{2}=0$. The weights correspond to the fractions of individuals with $T_{2}=1$ and $T_{2}=0$, which are determined by the experimental design. Alternatively, $\beta_{1}^{s}$ can be written as the sum of the ATE of $T_{1}$ relative to a counterfactual where $T_{2}=0$ and the interaction effect multiplied by the fraction of individuals with $T_{2}=1$; see Equation (9). Equations (10) and (11) present the corresponding expressions for $\beta_{2}^{s}$.

Unless the interaction effect is zero (in which case $\beta_{1}=\beta_{1}^{s}$ and $\beta_{2}=\beta_{2}^{s}$ ), the population regression coefficients in the short regression model neither correspond to the main effects nor to the interaction effect. Instead, the short model yields a composite treatment effect that is a weighted average of ATEs relative to different counterfactuals. ${ }^{10}$

\footnotetext{
${ }^{10}$ From a theoretical perspective, the choice between the long and the short model is related to the problem of making inference on a single treatment effect with covariates, where one has to decide whether to include the covariates linearly and to make inference on a weighted average of treatment effects or to run fully saturated regressions and to make inference on the ATE (e.g., Angrist \& Krueger, 1999; Angrist \& Pischke, 2009). However, the practical implications are not the same because experimental treatments are fundamentally different in nature from standard covariates; see Section 2.3 for a discussion.
} 


\subsection{Long or short model: What do we care about?}

Section 2.2 shows that the long model identifies the main effects relative to a businessas-usual counterfactual, whereas the short model yields a weighted average of treatment effects that depends on the nature and distribution of the other treatment arms in the experiment. However, this weighted average is typically neither of primary academic interest nor policy-relevant. This view is consistent with how papers we reanalyze motivate their object of interest, which is usually the main treatment effect against a business-as-usual counterfactual. Of the 19 papers in Table A.1 in Appendix A.1 that present results from the short model without all interactions, we did not find any study that mentioned (in the main text or in a footnote) that the presented treatment effects should be interpreted as either (a) a composite effect that includes a weighted average of the interaction with the other treatments or (b) as being against a counterfactual that was not business-as-usual but one that also had the other treatments in the same experiment.

One way to make the case for the short model is to recast the problem we identify as one of external rather than internal validity. Specifically, all experiments are carried out in a context with several unobserved "background" covariates. Thus, any experimental treatment effect is a weighted average of the treatment interacted with a distribution of unobserved covariates. If the other experimental arms are considered as analogous to unobserved background covariates, then inference on treatment effects based on the short model can be considered internally valid. In this view, the challenge is that the unobserved covariates (including other treatment arms) will vary across contexts.

However, experimental treatments are fundamentally different in nature from standard background covariates. They are determined by the experimenter based on research interest, and rarely represent real-world counterfactuals. In some cases, the interventions studied are new and the other treatments may not even exist in the study population. Even if they do exist, there is no reason to believe that the distributions in the population mirror those in the experiment. Thus, we view this issue as a challenge to internal validity because the other experimental arms are also controlled by the researcher and not just a set of "background unobservable factors". Further, papers with factorial designs often use the two-step procedure described in Section 4, and present results from the short model after mentioning that the interactions are not significantly different from zero (see for example, Banerjee et al. (2007) and Karlan \& List (2007)). This suggests that our view that interactions matter for internal validity is shared broadly.

Further, even in settings where the coefficients in the short model are of interest, they can always be constructed based on the coefficients in the long model, while the converse is not true. One can also use the long model to test hypotheses about the coefficients 
in the short regression model: $H_{0}: \beta_{1}^{s}=\beta_{1}+\beta_{12} P\left(T_{2}=1\right)=0$. Which test is more powerful depends on the relative sample size in the four experimental cells. ${ }^{11}$ Unlike the short model, the long model additionally allows for testing a rich variety of hypotheses about counterfactual effects such as $H_{0}: \beta_{1}+\beta_{12} p=0$ for policy-relevant values of $p$, which generally differ from the experimental assignment probability $P\left(T_{2}=1\right) .{ }^{12}$

To summarize, the long model estimates all the underlying parameters of interest (the main effects and the interactions). In contrast, $\beta_{1}^{s}$ is rarely of interest in its own right, and even if it is, the long model allows for estimation and inference on $\beta_{1}^{s}$ as well.

\subsection{Estimation and inference}

Suppose that the researcher has access to a random sample $\left\{Y_{i}, T_{1 i}, T_{2 i}\right\}_{i=1}^{N}$. Consider a factorial design with sample sizes as in Table 1 . In what follows, we focus on $\beta_{1}$. The analysis for $\beta_{2}$ is symmetric and omitted.

Under random assignment and standard regularity conditions, the OLS estimator of $\beta_{1}$ based on the long regression model, $\hat{\beta}_{1}$, is consistent:

$$
\hat{\beta}_{1} \stackrel{p}{\rightarrow} \beta_{1}=E\left(Y_{1,0}-Y_{0,0}\right)
$$

By contrast, the probability limit of the OLS estimators based on the short model is

$$
\hat{\beta}_{1}^{s} \stackrel{p}{\rightarrow} \beta_{1}^{s}=\beta_{1}+\beta_{12} P\left(T_{2}=1\right) .
$$

Unless the true interaction effect is zero (i.e., $\beta_{12}=0$ ), $\hat{\beta}_{1}^{s}$ is not consistent for the main effect relative to a business-as-usual counterfactual. Thus, if the goal is to achieve consistency for the main effects, one should always use the long model.

The choice between the long and the short regression model is less clear cut when it comes to inference. To illustrate, suppose that the data generating process is given by

$$
Y_{i}=\beta_{0}+\beta_{1} T_{1 i}+\beta_{2} T_{2 i}+\beta_{12} T_{1 i} T_{2 i}+\varepsilon_{i}
$$

\footnotetext{
${ }^{11}$ For example, when $N_{1}=N_{2}=N_{3}=N_{4}=N / 4$, the tests based on the long model and the short model exhibit the same power. In practice, we recommend comparing both tests when doing power calculations. If both tests have the same power, implementation based on the short model is more straightforward.

${ }^{12}$ For instance, resume audit experiments may vary characteristics such as age, gender, race, education, and experience with the sample size allocated to various combinations of these characteristics being different from their proportion in the population. In such a case, short model estimates are difficult to interpret; whereas, estimating the long model and calculating a weighted average of main and interaction effects with weights equal to their population proportions may yield a more policy-relevant treatment effect.
} 
where $\varepsilon_{i} \sim N\left(0, \sigma^{2}\right)$ is independent of $\left(T_{1 i}, T_{2 i}\right)$ and $\sigma^{2}$ is known. Normality allows us to formally compute and compare the finite sample power of the $t$-tests based on the short and the long regression model.

If the interaction effect is zero (i.e., $\beta_{12}=0$ ), it follows from standard results that, conditional on $\left(T_{11}, \ldots, T_{1 N}, T_{21}, \ldots, T_{2 N}\right)$,

$$
\hat{\beta}_{1} \sim N\left(\beta_{1}, \operatorname{Var}\left(\hat{\beta}_{1}\right)\right) \text { and } \hat{\beta}_{1}^{s} \sim N\left(\beta_{1}, \operatorname{Var}\left(\hat{\beta}_{1}^{s}\right)\right),
$$

where

$$
\operatorname{Var}\left(\hat{\beta}_{1}^{s}\right) \leq \operatorname{Var}\left(\hat{\beta}_{1}\right) .
$$

As a consequence, the $t$-test based on the short model exhibits higher finite sample power than the $t$-test based on the long model. Appendix A.3 gives explicit formulas of $\operatorname{Var}\left(\hat{\beta}_{1}\right)$ and $\operatorname{Var}\left(\hat{\beta}_{1}^{s}\right)$ in terms of $\left(N_{1}, N_{2}, N_{3}, N_{4}\right)$, provides a formal comparison between the power of the long and the short model, and discusses the role of the "size" of the interaction cell, $N_{4}$.

If, on the other hand, the interaction effect is not zero (i.e., $\beta_{12} \neq 0$ ), ignoring the interaction can lead to substantial size distortions as we demonstrate in Section 3.1. Depending on the true value of the interaction effect, the finite sample power of the $t$-test based on the short model can be higher or lower than the power of the $t$-test based on the long model.

\section{Factorial designs in practice}

In this section we document common practices among researchers studying field experiments with factorial designs. We analyze all articles published between 2007 and 2017 in the top five journals in Economics. ${ }^{13}$ Of the 3,505 articles published in this period $124(3.5 \%)$ are field experiments (Table A.1 provides more details). Factorial designs are widely used: Among 124 field experiments $27(22 \%)$ had a factorial design. ${ }^{14}$ Only

\footnotetext{
${ }^{13}$ These journals are The American Economic Review, Econometrica, The Journal of Political Economy, The Quarterly Journal of Economics, and The Review of Economic Studies. We exclude the May issue of the American Economic Review, known as "AER: Papers and Proceedings".

${ }^{14}$ We do not consider two-stage randomization designs as factorial designs. A two-stage randomization design is where some treatment is randomly assigned in one stage. In the second stage, treatment status is re-randomized to study behavioral changes conditional on a realization of the previous treatment. Examples of studies with two-stage randomization designs include Cohen \& Dupas (2010), Karlan \& Zinman (2009), and Ashraf et al. (2010). Finally, we do not include experiments where there is no "treatment", but rather conditions are randomized to elicit individuals preference parameters (e.g., Andersen et al., 2008; Gneezy et al., 2009; Fisman et al., 2008).
} 
8 of these 27 articles with factorial designs ( $30 \%)$ used the long model including all interaction terms as their main specification (see Table 2).

Table 2: Field experiments published in top-5 journals between 2007 and 2017

\begin{tabular}{lcccccc}
\hline & AER & ECMA & JPE & QJE & ReStud & Total \\
\hline Field experiments & 43 & 9 & 14 & 45 & 13 & 124 \\
With factorial designs & 11 & 2 & 4 & 6 & 4 & 27 \\
\hline Interactions included & 3 & 1 & 1 & 2 & 1 & 8 \\
Interactions not included & 8 & 1 & 3 & 4 & 3 & 19 \\
\hline
\end{tabular}

\subsection{Ignoring the interaction: Theory}

The discussion above highlights that it is common for experimental papers with factorial designs to ignore the interaction and focus on the short regression model. This is theoretically justified if the researcher is certain that all the interactions are zero, in which case it leads to consistent estimates of the main effects and to power improvements relative to the long model (see Section 2.4). However, if the interactions are not zero, ignoring the interaction yields inconsistent estimates and size distortions.

To illustrate, we introduce a running example based on a prototypical setting which we will return to throughout the paper. We focus on the problem of testing the null hypothesis that the main effect of $T_{1}$ is equal to zero, $H_{0}: \beta_{1}=0$. The analysis for $\beta_{2}$ is symmetric and omitted. We consider a $2 \times 2$ design with a total sample size of $N=1,000$, where $N_{1}=N_{2}=N_{3}=N_{4}=250$. The data are generated as

$$
Y_{i}=\beta_{1} T_{1 i}+\beta_{2} T_{2 i}+\beta_{12} T_{1 i} T_{2 i}+\varepsilon_{i}, \quad \varepsilon_{i} \sim N(0,1),
$$

where $T_{1 i}$ and $T_{2 i}$ are randomly assigned treatments with $P\left(T_{1 i}=1\right)=P\left(T_{2 i}=1\right)=0.5$. This experiment has power $90 \%$ to detect an effect size of $0.2 \sigma$ at the $5 \%$ level using the short regression. ${ }^{15}$ We use Monte Carlo simulations to assess the rejection rates of different inference procedures under the null (size) and the alternative hypothesis (power).

\footnotetext{
${ }^{15}$ The minimum detectable effect for the long model with power $90 \%$ and size $5 \%$ is $0.29 \sigma$.
} 
Figure 1 shows how power, bias, and size vary across different values of $\beta_{12}$ in both the long and the short model. When $\beta_{12}$ is exactly zero, the short model controls size and exhibits higher power than the long model. However, these power gains come at the cost of bias and size distortions whenever $\beta_{12} \neq 0$. As seen in Figure 1 , even modest values of $\left|\beta_{12}\right|$ lead to considerable size distortions. For instance, a $\left|\beta_{12}\right|$ greater than $0.1 \sigma$ (which occurs in over $36 \%$ of cases in the data we reanalyze) would more than double the rate of false rejection of the null. By contrast, the long model is unbiased and exhibits correct size for all values $\beta_{12}$. The main takeaway from Figure 1 is that researchers should avoid the short model, unless there is no uncertainty that $\beta_{12}=0$.

Figure 1: The perceived power gains from the short model come at the cost of biased estimators and not controlling size, unless $\beta_{12}$ is exactly equal to zero

(a) Power

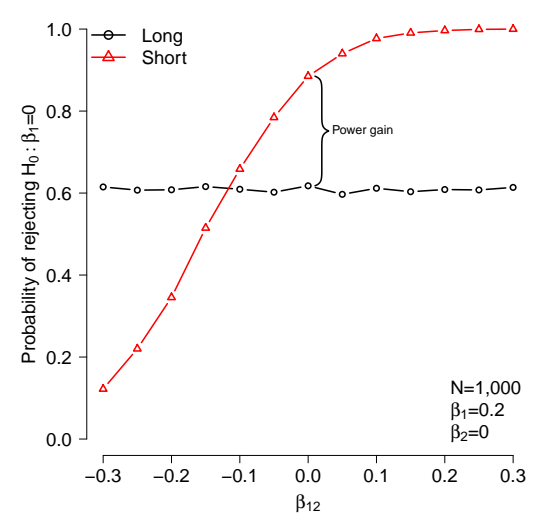

(b) Bias

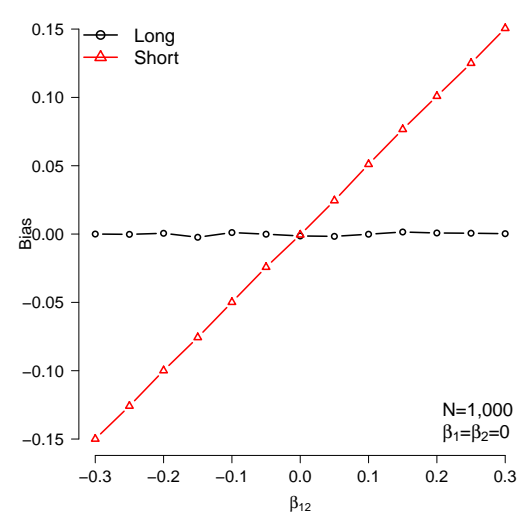

(c) Size

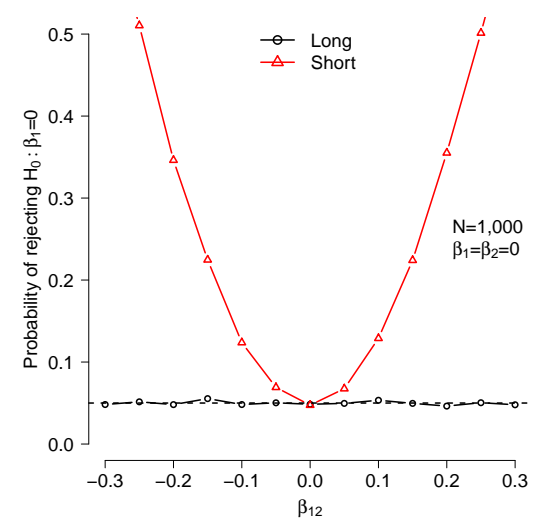

Note: Simulations are based on sample size $N$, normal iid errors, and 10,000 repetitions. The size for figures $1 c$ and $1 a$ is $\alpha=0.05$.

\subsection{Ignoring the interaction: Practice}

Here, we examine the practical implications of ignoring the interactions in the papers listed in Table A.1. We reanalyze the data from all field experiments with factorial designs and publicly available data that do not include all the interactions in the main specification. Of the ten most-cited papers with factorial designs listed in Table A.1 only one includes all the interactions in the main specification. More recent papers (which are less likely to be among the most cited) are more likely to include all interaction terms. Out of the 27 papers with factorial designs published in top-5 journals, 19 papers do not include all interaction terms. Of these 19, 4 papers did not have publicly-available replication data. In an online appendix we describe the experimental design of each of 
the 27 papers. ${ }^{16}$

We downloaded the publicly-available data files and replicated the main results in each of the remaining 15 papers. We standardized the outcome variable in each paper to have mean zero and standard deviation of one. We then compared the original treatment effects (estimated without the interaction terms) with those estimated including the interaction terms. In other words, we compare estimates based on the short model (Equation (4)) to those based on the long model (Equation (3)).

\subsubsection{Key facts about interactions}

As the discussion above highlights, the extent to which the short model will not control size depends on the value of the interactions in practice. We therefore start by plotting the distribution of estimated interaction effects (Figure 2) and documenting facts regarding interactions from our reanalysis. We find that interactions are quantitatively important and typically not second-order. All estimates are measured in standard deviations $(\sigma)$ of the outcome variable. While the median (mean) interaction for these papers is $0.00 \sigma(0.00 \sigma)$, the median (mean) absolute value of the interaction is $0.07 \sigma(0.13 \sigma)$. The median (mean) absolute value of interactions relative to the main treatment effects is 0.37 (1.55). Thus, while it may be true that interactions are small on average across all studies, they are often sizeable in any given study. In our data, the absolute value of the interactions is greater than $0.1 \sigma$ in $36 \%$ and greater than $0.2 \sigma$ in $19 \%$ of the cases. These lead to a $13 \%$ and $22 \%$ chance of rejecting the null of no effect in our running example (as seen in Figure 1), which corresponds to more than a doubling and a quadrupling, respectively, in the rate of false rejection at the $5 \%$ level.

The second key finding is that despite the interactions being quantitatively important, most experiments will rarely reject the null hypothesis that they are zero (Figure 2 shades the fraction of the interactions that are significant in the studies that we reanalyze). Among the 15 papers that we reanalyzed, $6.2 \%$ of interactions are significant at the $10 \%$ level, $3.6 \%$ are significant at the $5 \%$ level, and $0.9 \%$ are significant at the $1 \%$ level. ${ }^{17}$ These findings are not surprising because factorial designs are rarely powered to detect meaningful interactions. Section 7 provides a discussion of the issues related to using factorial designs for making inferences on interaction effects.

The fact that most experiments were not explicitly powered to detect interactions suggests that the main reason for running experiments with factorial designs seems to be

\footnotetext{
${ }^{16}$ Available at http://mauricio-romero.com/pdfs/papers/Appendix_crosscuts.pdf

${ }^{17}$ Among the papers that originally included all interactions, $4.5 \%$ of interactions are significant at the $10 \%$ level, $1.1 \%$ are significant at the $5 \%$ level, and $0.0 \%$ are significant at the $1 \%$ level.
} 
the increase in power for detecting main effects. However, as we show below, this comes at the considerable cost of an increased rate of false positives (which is unsurprising based on the distribution of interactions shown in Figure 2).

Figure 2: Distribution of the estimated interaction effects

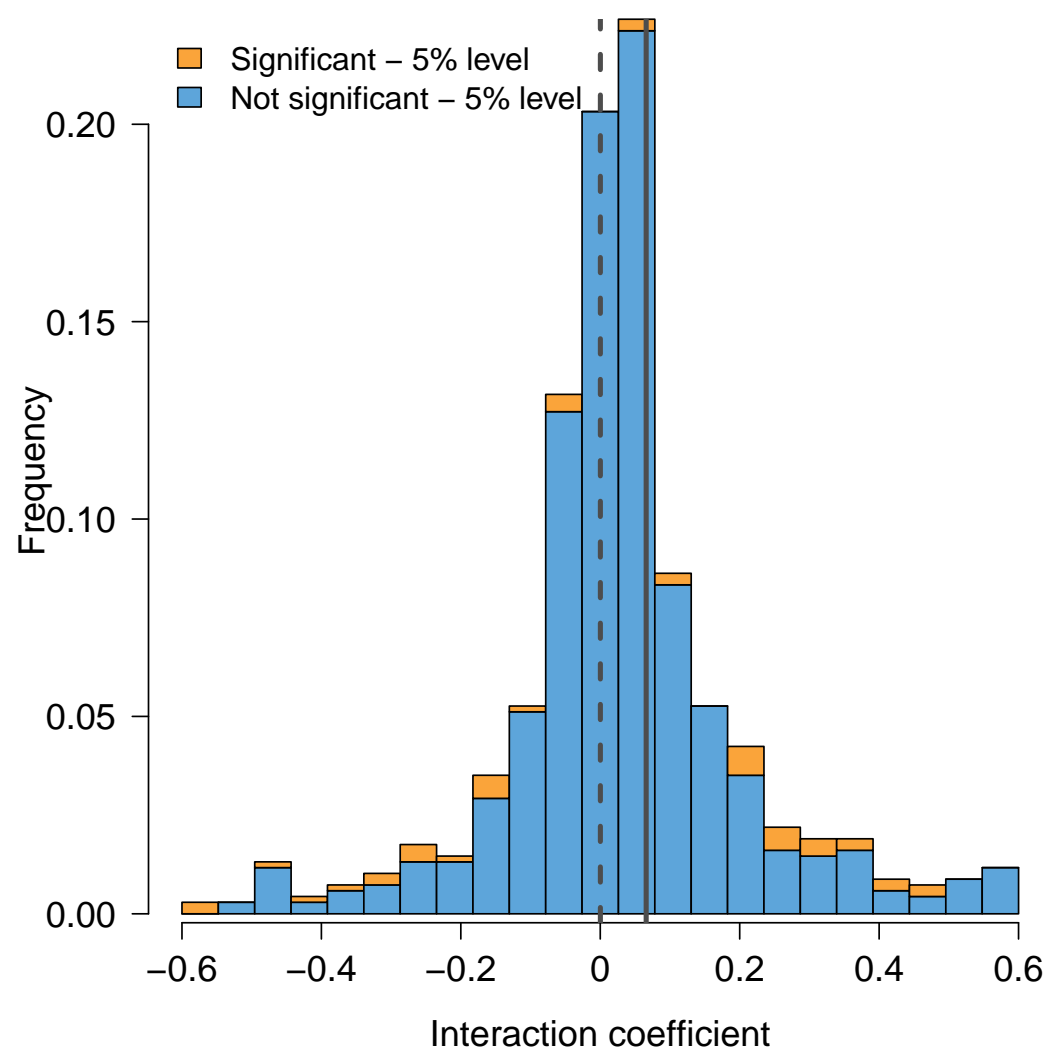

Note: This figure shows the distribution of the interactions between the main treatments. We trim the top and bottom $1 \%$ of the distribution. The median interaction for these papers is $0.00 \sigma$ (dashed vertical line), the median absolute value of the interaction is $0.07 \sigma$ (solid vertical line), and the median relative absolute value of the interaction with respect to the main treatment effect is $0.37 .6 .2 \%$ of interactions are significant at the $10 \%$ level, $3.6 \%$ are significant at the $5 \%$ level, and $0.9 \%$ are significant at the $1 \%$ level.

\subsubsection{Implications of ignoring interactions}

Figure 3a compares the original treatment effect estimates based on the short model to the estimates based on the long model which includes the interaction terms (Figure $3 \mathrm{~b}$ zooms in to cases where the value of the main treatment effects in the short model is between -1 to 1 standard deviation). The median change in the absolute value of the point estimate of the main treatment effect is $96 \%$. Roughly $26 \%$ of estimated treatment effects change sign when they are estimated using the long regression. 
Table 3 shows how the significance of the main treatment estimates changes when using the long instead of the short model. About $48 \%$ of treatment estimates that were significant at the $10 \%$ level based on the short model are no longer significant based on the long model. $53 \%$ and $57 \%$ of estimates lose significance at the $5 \%$ and $1 \%$ levels, respectively. A much smaller fraction of treatment effects that were not significant in the short model are significant based on the long regression $(6 \%, 5 \%$, and $1 \%$, at the $10 \%$, $5 \%$, and $1 \%$ levels respectively).

We find similar results when we restrict our reanalysis to the ten most cited papers with factorial designs that do not include the interaction terms (with data available for reanalysis). When we re-estimate the treatment effects in these papers after including all interactions, we find that out of 21 results that were significant at the $5 \%$ level in the paper, 9 (or $43 \%$ ) are no longer so after including all interactions. Corresponding figures and tables are presented in Appendix A.1.2 (Figure A.2 and Table A.2).

Finally, we also distinguish between policy and conceptual experiments in Table A.1 (the latter typically have more treatments and interactions) and see that the problem of incorrect inference from ignoring interaction terms remains even when we restrict attention to the policy experiments. Of the 12 policy experiments, 9 do not include all interactions. When we re-estimate the treatment effects in these 9 papers after including all interactions, we find that out of 19 results that were significant at the $5 \%$ level in the paper, 6 (or 32\%) are no longer so after including all interactions. Corresponding figures and tables are presented in Appendix A.1.3 (Figure A.4 and Table A.3). 
Figure 3: Treatment estimates based on the long and the short model

(a) All estimates

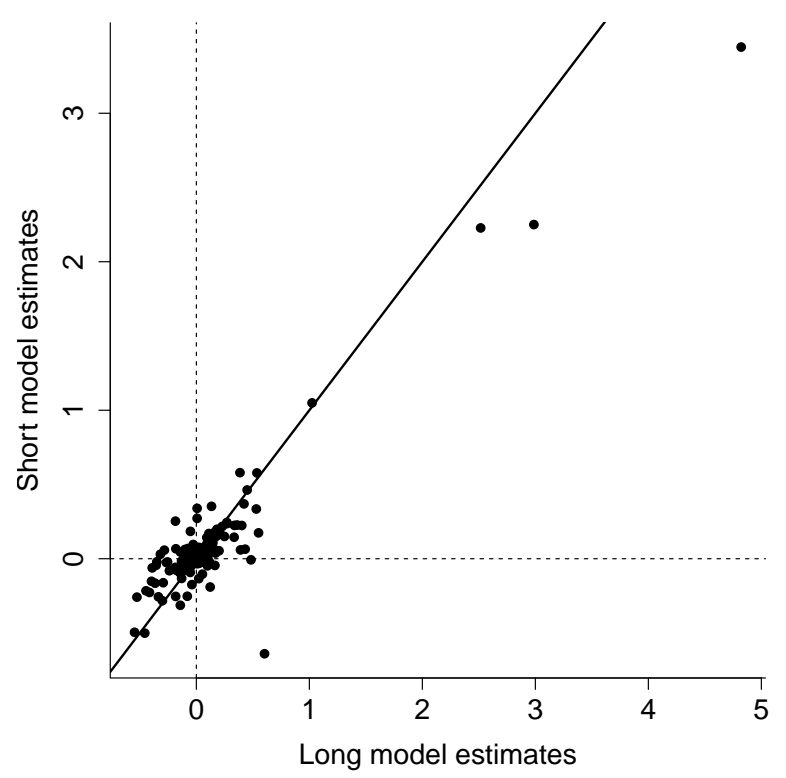

(b) Main treatment effects between $-1 \sigma$ and $1 \sigma$

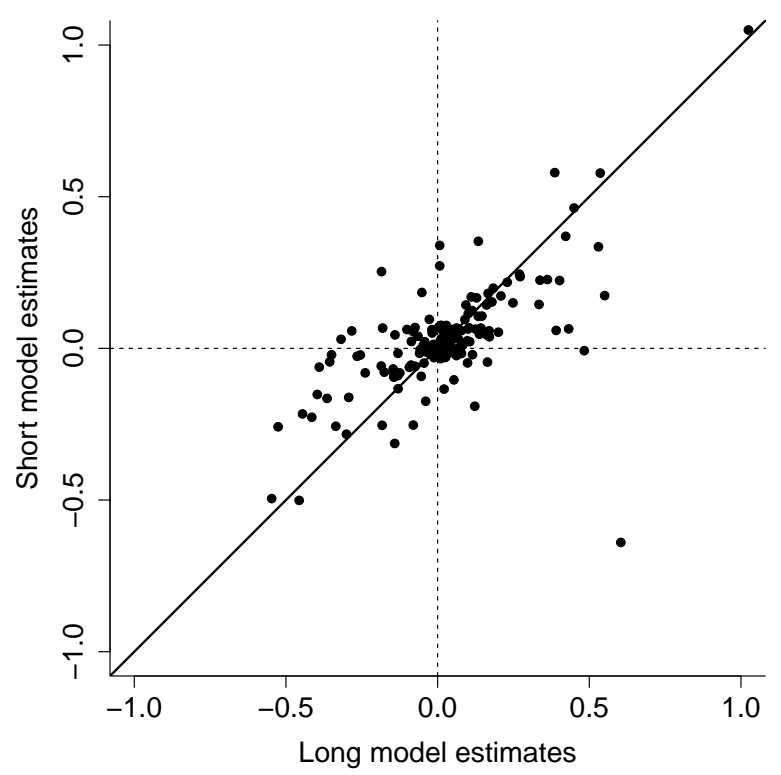

Note: This figure shows how the main treatment estimates change between the short and the long model across all studies. Figure $3 a$ has all the treatment effects, while Figure $3 b$ zooms in to cases where the value of the main treatment effects in the short model is between -1 to 1 standard deviation. The median main treatment estimate from the short model is $0.01 \sigma$, the median main treatment estimate from the long model is $0.02 \sigma$, the average absolute difference between the treatment estimates of the short and the long model is $0.05 \sigma$, the median absolute difference in percentage terms between the treatment estimates of the short and the long model is $96 \%$, and $26 \%$ of treatment estimates change sign when they are estimated using the long model instead of the short model. 
Table 3: Significance of treatment estimates based on the long and the short model

\begin{tabular}{|c|c|c|c|}
\hline \multirow[b]{2}{*}{ With interaction } & \multicolumn{2}{|c|}{ Without interaction } & \multirow[b]{2}{*}{ Total } \\
\hline & Not significant & Significant & \\
\hline Not significant & 95 & 34 & 129 \\
\hline Significant & 6 & 37 & 43 \\
\hline Total & 101 & 71 & 172 \\
\hline \multicolumn{4}{|c|}{ Panel B: Significance at the $5 \%$ level } \\
\hline & \multicolumn{2}{|c|}{ Without interaction } & \\
\hline With interaction & Not significant & Significant & Total \\
\hline Not significant & 111 & 29 & 140 \\
\hline Significant & 6 & 26 & 32 \\
\hline Total & 117 & 55 & 172 \\
\hline \multicolumn{4}{|c|}{ Panel C: Significance at the $1 \%$ level } \\
\hline & \multicolumn{2}{|c|}{ Without interaction } & \\
\hline With interaction & Not significant & Significant & Total \\
\hline Not significant & 140 & 17 & 157 \\
\hline Significant & 2 & 13 & 15 \\
\hline Total & 142 & 30 & 172 \\
\hline
\end{tabular}

This table shows the number of coefficients that are significant at a given level when estimating the long regression (columns) and the short regression (rows). This table includes information from all papers with factorial designs and publicly available data that do not include the interactions in the original study. Panel A uses a 10\% significance level, Panel B uses 5\%, and Panel $\mathrm{C}$ uses $1 \%$.

\section{Model selection (or pre-testing) yields invalid inferences}

As implied by the quote from Kremer (2003) in the introduction, researchers often recognize that using the short model is only correct for inference on the main treatment effect if the interaction is close to zero. However, the problem is that the value of the interaction is not known ex ante. Therefore, a common practice is to employ a data-driven two-step procedure to determine whether to estimate the full model or to ignore the interaction. Specifically, the steps are:

1. Estimate the long model and test the null hypothesis that $\beta_{12}$ is zero (i.e., $H_{0}: \beta_{12}=$ 0 ) using a two-sided $t$-test. 
2. (a) If $H_{0}: \beta_{12}=0$ is rejected, test $H_{0}: \beta_{1}=0$ using the two-sided $t$-test based on the long model.

(b) If $H_{0}: \beta_{12}=0$ is not rejected, test $H_{0}: \beta_{1}=0$ using the two-sided $t$-test based on the short model.

It is well-known that the distributions of the estimators obtained from this data-dependent model selection procedure are complicated and highly non-normal, rendering the usual t-statistic-based inference invalid (e.g., Leeb \& Pötscher, 2005, 2006, 2008). To illustrate this issue, we return to our running example. The size properties of the two-step model selection approach are shown in Figure 4. For reference, we also include results for the $t$-tests based on the long and the short model. The main takeaway from Figure 4 is that model selection leads to incorrect inferences and false positives. Thus, researchers should always avoid it.

The performance of the model selection approach to determine whether one should run the short or the long model is particularly poor because field experiments are rarely powered to reject that the interactions are zero (see Section 7). Figure 2 shows that only $3.6 \%$ of interactions were significant at the $5 \%$ level in our reanalysis. Using a rejection threshold of $5 \%$, the model-selection approach would lead to estimating the short model in over $96 \%$ of the cases we reanalyze. Thus, the rate of incorrect inference under modelselection will continue to be nearly as high as just running the short model. 
Figure 4: Model selection does not control size

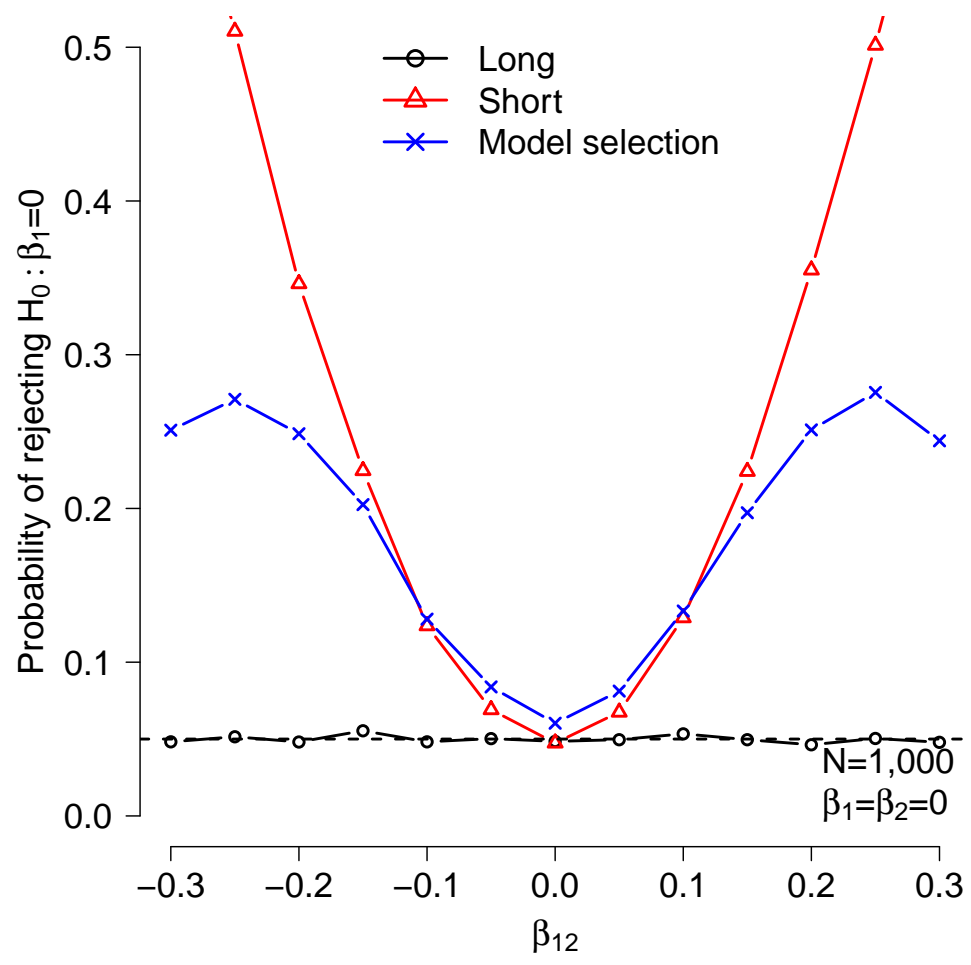

Note: Simulations are based on sample size $N$, normal iid errors, and 10,000 repetitions. The size is $\alpha=0.05$. For the model selection, the short model is estimated if one fails to reject $\beta_{12}=0$ at the $5 \%$ level.

\section{Can we improve power for detecting main effects while achieving size control?}

We now examine whether it is possible to improve power for detecting main effects relative to $t$-tests based on the long model, while maintaining size control for relevant values of the interactions. We first consider $2 \times 2$ factorial designs, and discuss factorial designs with more than two treatments in Section 6.

To simplify the exposition, we focus on $\beta_{1}$ and partial out $T_{2}$ and the constant, keeping the partialling-out implicit. The analysis for $\beta_{2}$ is symmetric and omitted. Defining $T_{12} \equiv T_{1} T_{2}$, the regression model of interest is ${ }^{18}$

$$
Y=\beta_{1} T_{1}+\beta_{12} T_{12}+\varepsilon .
$$

\footnotetext{
${ }^{18}$ We omit the intercept because all variables have mean zero after partialling-out $T_{2}$ and the constant.
} 
Our goal is to test hypotheses about the main effect $\beta_{1}$.

\subsection{Optimality properties of the $t$-test based on the long model}

The two-sided $t$-test based on the long regression model is the uniformly most powerful test among tests that are unbiased for all values of the interaction effect (e.g., van der Vaart, 1998). ${ }^{19}$ The practical implication of this classical result is that any procedure that is more powerful than the $t$-test for some values of the interaction must underperform somewhere else. As a consequence, to achieve higher power than the $t$-test based on the long model, one has to make a choice about which values of the interaction to direct power to. In practice, this choice needs to be made based on some form of prior knowledge.

The scope for power improvements relative to the two-sided $t$-test based on the long regression model is theoretically limited if one insists on uniform size control. The reason is that for the corresponding one-sided testing problem, the usual one-sided $t$ test based on the long model is the uniformly most powerful test among all tests (e.g., Proposition 15.2 in van der Vaart, 1998). Thus, at any parameter value, the uniformly most powerful test is a one-sided $t$-test and the best one can hope for is to improve the power from the two-sided to a one-sided test (see, e.g., Armstrong et al. (2019) and Armstrong \& Kolesar (2019) for a further discussion of this point). At the 5\%-level, this power improvement is never larger than 12.5 percentage points. It can also be shown that the scope for improving the average length of the usual confidence intervals based on the long regression model is limited (e.g., Armstrong \& Kolesar, 2018, 2019; Armstrong et al., 2019). ${ }^{20}$

Section 5.2 proposes a nearly optimal test which comes close to achieving the maximal power gain at a priori likely values of the interaction, while controlling size for all values of the interaction. In Section 5.3, we explore an approach based on prior knowledge on the magnitude of the interaction. When the prior knowledge is correct, this approach controls size and yields power gains relative to the $t$-test based on the long model. However, unlike the $t$-test based on the long model and the nearly optimal test, it suffers from size distortions if the prior knowledge is incorrect. Appendix A.5 explores two additional econometric approaches based on work by Imbens \& Manski (2004), Stoye (2009), and McCloskey (2017).

\footnotetext{
${ }^{19} \mathrm{~A}$ test is unbiased if its power is larger than its size.

${ }^{20}$ Moreover, the results in Joshi (1969) imply the usual two-sided confidence interval based on the long regression model achieves minimax expected length (Armstrong \& Kolesar, 2019).
} 


\subsection{Nearly optimal tests targeting power towards a likely value $\bar{\beta}_{12}$}

Consider a scenario where a particular value $\beta_{12}=\bar{\beta}_{12}$ seems a priori likely and suppose that we want to find a test that controls size and is as powerful as possible when $\beta_{12}=$ $\bar{\beta}_{12}$. For concreteness, we focus on the case where $\bar{\beta}_{12}=0$ and consider the following testing problem

$$
H_{0}: \beta_{1}=0, \beta_{12} \in \mathbb{R} \text { against } H_{1}: \beta_{1} \neq 0, \beta_{12}=0 .
$$

We use the numerical algorithm developed by Elliott et al. (2015) to construct a nearly optimal test for the testing problem (13).

To describe their procedure, note that under standard conditions, the $t$-statistics are approximately normally distributed in large samples

$$
\left(\begin{array}{c}
\hat{t}_{1} \\
\hat{t}_{12}
\end{array}\right) \sim N\left(\left(\begin{array}{c}
t_{1} \\
t_{12}
\end{array}\right),\left(\begin{array}{ll}
1 & \rho \\
\rho & 1
\end{array}\right)\right),
$$

where $\hat{t}_{1}=\frac{\hat{\beta}_{1}}{\operatorname{SE}\left(\hat{\beta}_{1}\right)}, \hat{t}_{12}=\frac{\hat{\beta}_{12}}{\operatorname{SE}\left(\hat{\beta}_{12}\right)}, t_{1}=\frac{\beta_{1}}{\operatorname{SE}\left(\hat{\beta}_{1}\right)}, t_{12}=\frac{\beta_{12}}{\operatorname{SE}\left(\hat{\beta}_{12}\right)}$, and $\rho=\operatorname{Cov}\left(\hat{t}_{1}, \hat{t}_{12}\right)$. We also define $\hat{t}=\left(\hat{t}_{1}, \hat{t}_{12}\right)$ and $t=\left(t_{1}, t_{12}\right)$. SE $\left(\hat{\beta}_{1}\right), S E\left(\hat{\beta}_{12}\right)$ and $\operatorname{Cov}\left(\hat{t}_{1}, \hat{t}_{12}\right)$ can be consistently estimated under weak conditions (here we use a standard heteroskedasticity robust estimator).

Consider the problem of maximizing power in the following hypothesis testing problem:

$$
H_{0}: t_{1}=0, t_{12} \in \mathbb{R} \text { against } H_{1}: t_{1} \neq 0, t_{12}=0 .
$$

A common approach to construct powerful tests for problems with composite hypotheses is to choose tests based on their weighted average power. In particular, we seek a

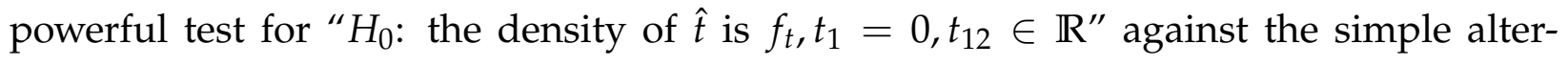
native " $H_{1, F}$ : the density of $\hat{t}$ is $\int f_{t} d F(t)$ ", where the weighting function $F$ is chosen by the researcher. Now suppose that the null is replaced by " $H_{0, \Lambda}$ : the density of $\hat{t}$ is $\int f_{t} d \Lambda(t)^{\prime \prime}$. To obtain the best test, one needs to find a least favorable distribution (LFD), $\Lambda^{L F}$, with the property that the size $\alpha$ Neyman-Pearson test of the simple hypothesis $H_{0, \Lambda}$ LF against $H_{1, F}$ also yields a size $\alpha$ test of the composite null hypothesis $H_{0}$ against $H_{1, F}$ (e.g., Lehmann \& Romano, 2005).

Since it is generally difficult to analytically determine and computationally approximate $\Lambda^{L F}$, Elliott et al. (2015) suggest to instead focus on an approximate LFD, $\Lambda^{A L F}$, 
which yields a nearly optimal test for $H_{0}$ against $H_{1, F}$. The resulting test is then just a Neyman-Pearson test based on $\Lambda^{A L F}$.

Figure 5: Elliott et al. (2015)'s nearly optimal test controls for size and yields power gains over running the full model for "intermediate" values of $\beta_{12}$
(a) Size
(b) Power
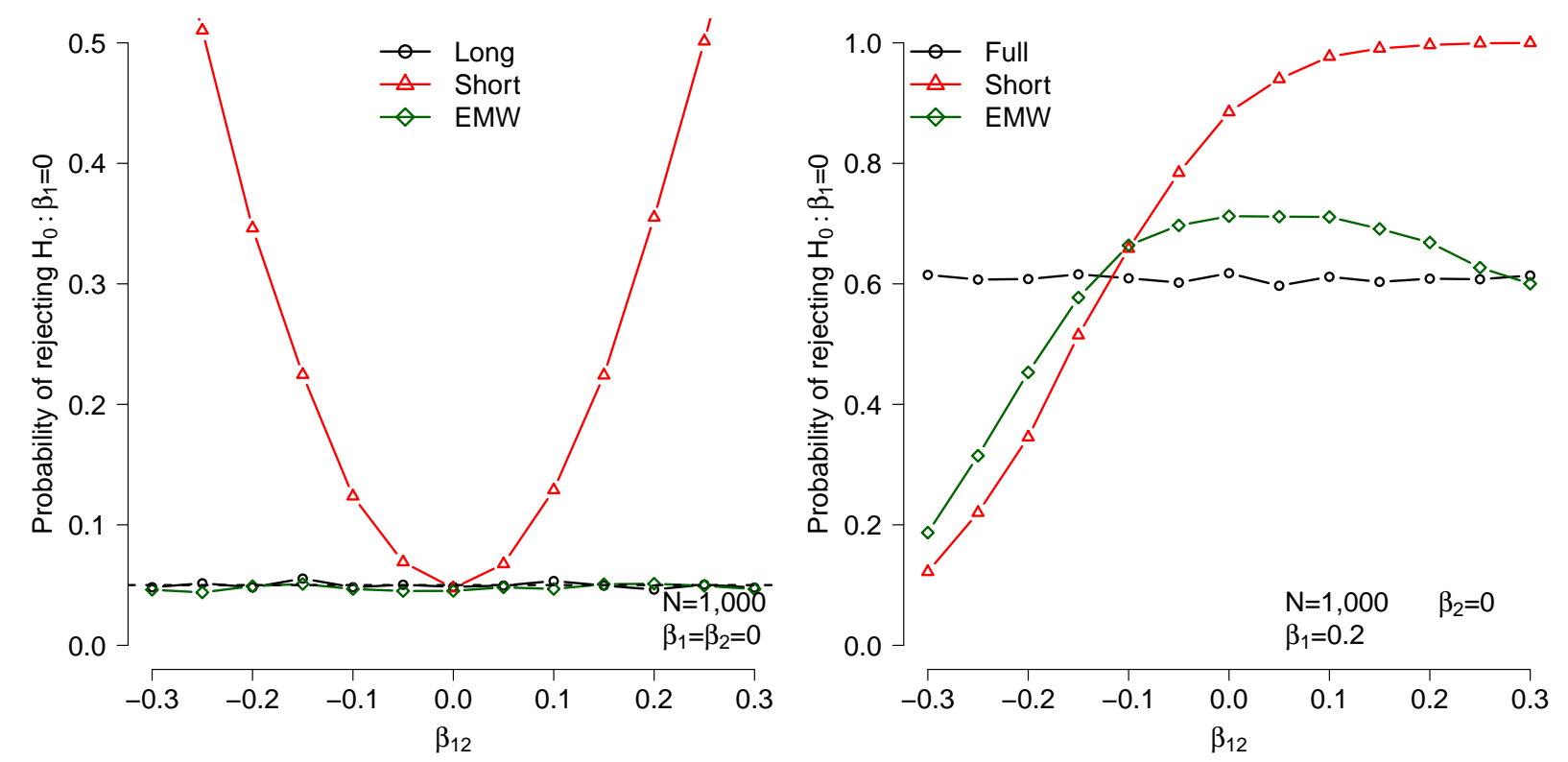

Note: Simulations are based on sample size $N$, normal iid errors, and 10,000 repetitions. The size for figures $5 a$ and $5 b$ is $\alpha=0.05$. EMW refers to Elliott et al. (2015)'s nearly optimal test.

Figure 5 displays the results of applying the nearly optimal test in the context of our running example. ${ }^{21}$ The test controls size for all values of $\beta_{12}$ and, by construction, is nearly optimal when $\beta_{12}=0$. A comparison with the $t$-test based on the long model shows that the nearly optimal test is more powerful when $\beta_{12}$ is close to zero. The nearly optimal test comes close to achieving the maximal possible power. For example, when $\beta_{1}=0.2$ the power of the nearly optimal test is $98.5 \%$ of the power of the one-sided $t$-test.

However, as expected given the discussion in Section 5.1, these power gains come at a cost. For certain values of $\beta_{12}$, the power can be much lower than that of the $t$-test based on the long model. Appendix A.6.3 provides a more comprehensive assessment of the performance of the nearly optimal tests by plotting power curves for different values of $\beta_{1}$.

\footnotetext{
${ }^{21}$ To improve the performance of their procedure, Elliott et al. (2015) suggest a switching rule that depends on $\left|\hat{t}_{12}\right|$ such that for large enough values of $\left|\hat{t}_{12}\right|$, one switches to regular hypothesis testing. Following their suggestion, we use 6 as the switching value.
} 


\subsection{Inference under a priori restrictions on the magnitude of $\beta_{12}$}

Suppose that the researcher is certain that $\beta_{12}=\bar{\beta}_{12}$. In this case, she can obtain powerful tests based on a regression of $Y-\bar{\beta}_{12} T_{12}$ on $T_{1}$. If $\bar{\beta}_{12}=0$, this corresponds to estimating the short model. As shown in Section 2.4, the $t$-test based on the short model is more powerful than the $t$-test based on the long model when the prior knowledge that $\beta_{12}=0$ is correct, but does not control size when it is not.

Of course, exact knowledge of $\beta_{12}$ may be too strong of an assumption. Suppose instead that the researcher imposes prior knowledge in the form of a restriction on the magnitude of the interaction effect $\beta_{12}$.

Assumption 1. $\left|\beta_{12}\right| \leq C$ for some finite constant $C$.

Assumption 1 restricts the parameter space for $\beta_{12}$ and implies that

$$
\beta_{12} \in\left\{b_{12}:\left|b_{12}\right| \leq C\right\} \equiv \mathcal{B}_{12}
$$

Here we use the approach developed in Armstrong \& Kolesar (2018) and Armstrong et al. (2019) to construct optimal confidence intervals under Assumption 1.22 To describe their procedure, we write model (12) in matrix form as

$$
\mathbf{Y}=\beta_{1} \mathbf{T}_{1}+\beta_{12} \mathbf{T}_{12}+\varepsilon
$$

and assume that $\varepsilon \sim N\left(0, \sigma^{2} \mathbf{I}_{N}\right)$ and $\sigma^{2}$ is known. The implementation with heteroskedastic and non-Gaussian errors is discussed in Appendix A.4. An affine estimator of $\beta_{1}$ can be written as $\hat{\beta}_{1}=a+b^{\prime} \mathbf{Y}$, for some $a$ and $b$ that can depend on $\mathbf{X} \equiv\left(\mathbf{T}_{1}, \mathbf{T}_{12}\right)$. For example, for the long OLS regression model, $a=0$ and $b$ is the first row of $\left(\mathbf{X}^{\prime} \mathbf{X}\right)^{-1} \mathbf{X}^{\prime}$.

Define the "worst case" biases as

$$
\begin{aligned}
& \overline{\operatorname{Bias}}\left(\hat{\beta}_{1}\right)=\sup _{\beta_{1} \in \mathbb{R}, \beta_{12} \in \mathcal{B}_{12}} E_{\left(\beta_{1}, \beta_{12}\right)}\left(\hat{\beta}_{1}-\beta_{1}\right), \\
& \underline{\operatorname{Bias}}\left(\hat{\beta}_{1}\right)=\inf _{\beta_{1} \in \mathbb{R}, \beta_{12} \in \mathcal{B}_{12}} E_{\left(\beta_{1}, \beta_{12}\right)}\left(\hat{\beta}_{1}-\beta_{1}\right),
\end{aligned}
$$

where $E_{\left(\beta_{1}, \beta_{12}\right)}$ denotes the expectation under model (16) with $\left(\beta_{1}, \beta_{12}\right)$. Assuming that $\left(\mathbf{T}_{1}, \mathbf{T}_{12}\right)$ are fixed, $\hat{\beta}_{1}$ is normally distributed with mean $a+b^{\prime}\left(\beta_{1} \mathbf{T}_{1}+\beta_{12} \mathbf{T}_{12}\right)$ and variance $S E\left(\hat{\beta}_{1}\right)^{2}=\|b\|_{2}^{2} \sigma^{2}$. Thus, as $\left(\beta_{1}, \beta_{12}\right)$ varies over $\mathbb{R} \times \mathcal{B}_{12}$, the $t$-ratio, $\frac{\left(\hat{\beta}_{1}-\beta_{1}\right)}{\operatorname{SE}\left(\hat{\beta}_{1}\right)}$,

\footnotetext{
${ }^{22}$ Optimality here refers to minimizing the width of the confidence intervals. We focus on the width of the confidence intervals because of the intuitive appeal and practical relevance of this criterion. If one were to optimize the power of the test that the confidence interval inverts, the resulting procedure can be different.
} 
is normally distributed with variance one and mean varying from $\frac{\operatorname{Bias}\left(\hat{\beta}_{1}\right)}{S E\left(\hat{\beta}_{1}\right)}$ to $\frac{\overline{\operatorname{Bias}}\left(\hat{\beta}_{1}\right)}{\operatorname{SE}\left(\hat{\beta}_{1}\right)}$. To construct a two-sided confidence interval, note that testing $H_{0}: \beta_{1}=\beta_{1}^{0}$ based on a $t$-statistic with critical value $c v_{\alpha}\left(\frac{\max \left\{\left|\operatorname{Bias}\left(\hat{\beta}_{1}\right)\right|,\left|\overline{\operatorname{Bias}}\left(\hat{\beta}_{1}\right)\right|\right\}}{S E\left(\hat{\beta}_{1}\right)}\right)$ yields a level $\alpha$ test, where $c v_{\alpha}(t)$ denotes the $1-\alpha$ quantile of a folded normal distribution with location parameter $t$ and scale parameter 1 . Inverting this test yields the following confidence interval:

$$
\hat{\beta}_{1} \pm c v_{\alpha}\left(\frac{\max \left\{\left|\underline{\operatorname{Bias}}\left(\hat{\beta}_{1}\right)\right|,\left|\overline{\operatorname{Bias}}\left(\hat{\beta}_{1}\right)\right|\right\}}{S E\left(\hat{\beta}_{1}\right)}\right) S E\left(\hat{\beta}_{1}\right)
$$

The length of the confidence interval (17) is determined by the bias and the variance of the estimator $\hat{\beta}_{1}$, and to obtain optimal confidence intervals one has to solve a biasvariance trade-off. This problem is amenable to convex optimization and we describe how to solve it in Appendix A.4.

Figure 6 reports the rejection probabilities of a test that rejects if zero is not in the confidence interval. For the purpose of illustration, we consider $C=0.1$ such that $\mathcal{B}_{12}=[-0.1,0.1]$. Our results suggest that imposing prior knowledge in the form of an upper bound on the absolute value of the interaction effect can yield substantial power improvements relative to the $t$-tests based on the long regression model, while controlling size when this prior knowledge is in fact correct. Appendix A.6.4 presents the corresponding power curves for different values of $\beta_{1}$.

When researchers are primarily interested in the main effects, and feel confident that the interactions are second-order, Armstrong et al. (2019)'s approach should be strictly preferred to the short model, since it is more realistic to pre-specify that the interaction is in a range than exactly zero. However, pre-specifying the appropriate range of prior values for the interaction is non-trivial and requires judgment, because this approach still exhibits size distortions when the prior knowledge is incorrect (e.g., when $\left|\beta_{12}\right|>0.1$ in Figure 6). ${ }^{23}$

\footnotetext{
${ }^{23}$ Note that it is problematic to use Armstrong et al. (2019)'s approach based on first running the long model and not rejecting that the interaction is in a certain range. This would result in the same datadependent model selection issue that arises from reporting the short model after failing to reject that interactions are significantly different from zero in the long model. Thus, while Armstrong et al. (2019)'s approach is an improvement over the short model, it does not solve the underlying problem of not knowing the true value of the interaction.
} 
Figure 6: Restrictions on the magnitude of $\beta_{12}$ yield power gains if they are correct but lead to incorrect inferences if they are not

(a) Size

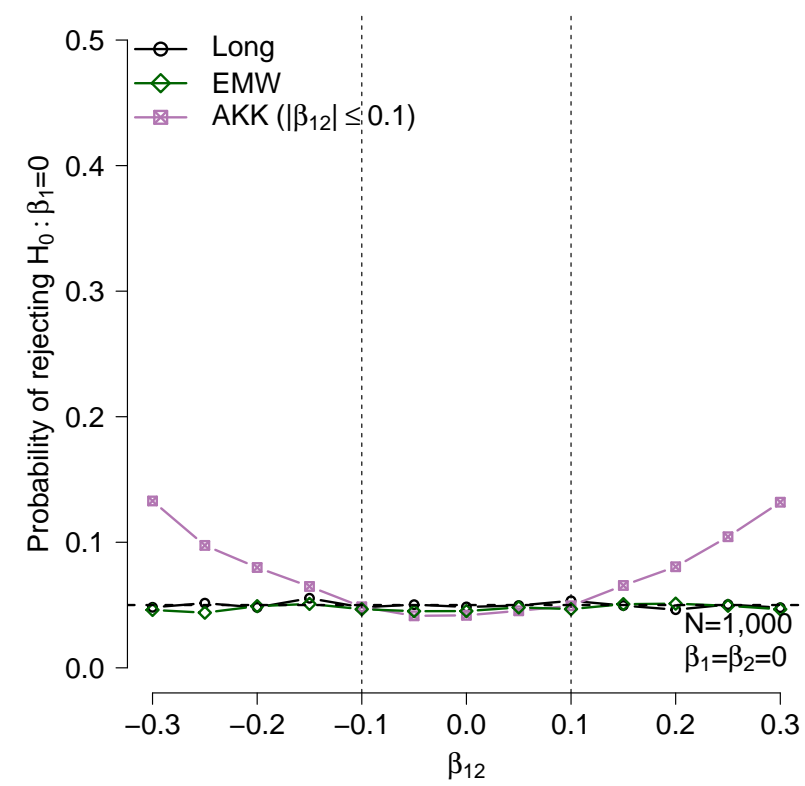

(b) Power

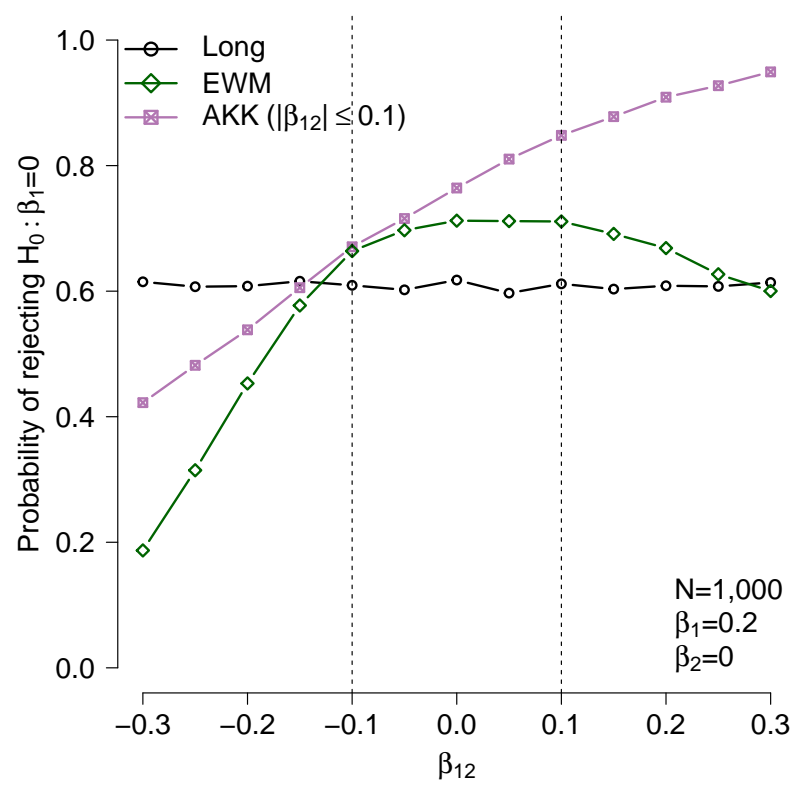

Note: Simulations are based on sample size $N$, normal iid errors, and 10,000 repetitions. The size for figures $6 a$ and $6 b$ is $\alpha=0.05$. AKK refers to Armstrong et al. (2019)'s approach for constructing optimal confidence intervals under prior knowledge about the magnitude of $\beta_{12}$ with $\mathcal{B}_{12}=[-0.1,0.1]$ (dashed vertical lines). EMW refers to Elliott et al. (2015)'s nearly optimal test.

\subsection{A design-based approach for improving power}

The discussion above focused on improving power for detecting main effects in existing experiments with factorial designs. For the design of new experiments, a design-based alternative is to leave the "interaction cell" empty (i.e., to set $N_{4}=0$ ) and to re-assign those subjects to the other cells such that

\begin{tabular}{|c|c|c|c|}
\cline { 3 - 4 } \multicolumn{2}{c|}{} & \multicolumn{2}{c|}{$T_{1}$} \\
\cline { 3 - 4 } \multicolumn{2}{c|}{} & No & Yes \\
\hline \multirow{2}{*}{$T_{2}$} & No & $N_{1}^{*}$ & $N_{2}^{*}$ \\
\cline { 2 - 4 } & Yes & $N_{3}^{*}$ & 0 \\
\hline
\end{tabular}

Consider the following regression model

$$
Y=\beta_{0}^{*}+\beta_{1}^{*} T_{1}+\beta_{2}^{*} T_{2}+\varepsilon^{*}
$$


Let $\hat{\beta}_{1}^{*}$ and $\hat{\beta}_{2}^{*}$ denote the OLS estimators of $\beta_{1}^{*}$ and $\beta_{2}^{*}$. We show in Appendix A.2.3 that if $T_{1}$ and $T_{2}$ are randomly assigned, $\hat{\beta}_{1}^{*}$ and $\hat{\beta}_{2}^{*}$ are consistent for the respective main effects.

To illustrate the power implications of leaving the interaction cell empty, consider an experiment where the researcher cares equally about power to detect an effect of $T_{1}$ and $T_{2}$, and thus assigns the same sample size to both treatments: $N_{2}^{*}=N_{3}^{*}=N_{T}^{*}$. In what follows, we focus on $\beta_{1}^{*}$. The analysis for $\beta_{2}^{*}$ is symmetric and omitted. Under the assumptions of Section A.3.1, the (conditional) variance of $\hat{\beta}_{1}^{*}$ is given by

$$
\operatorname{Var}\left(\hat{\beta}_{1}^{*}\right)=\sigma^{2} \frac{N-N_{T}^{*}}{\left(N-2 N_{T}^{*}\right) N_{T}^{*}} .
$$

$\operatorname{Var}\left(\hat{\beta}_{1}^{*}\right)$ is minimized when $N_{T}^{*}=\frac{N}{2}(2-\sqrt{2})$ and we assume that the experiment is designed in this manner. ${ }^{24}$ A comparison to the variance of the estimator based on the long model, $\hat{\beta}_{1}$, shows that $\operatorname{Var}\left(\hat{\beta}_{1}^{*}\right) \leq \operatorname{Var}\left(\hat{\beta}_{1}\right)$. Thus, by the same reasoning as in Section 2.4, leaving the interaction cell empty leads to power improvements for testing hypotheses about the main effects relative to the long regression model.

Figure 7 presents the results based on our running example. As expected, leaving the interaction cell empty yields tests that control size for all values of the interaction. Moreover, among the approaches that achieve size control for all values of $\beta_{12}$ (the long model and the nearly optimal test), leaving the interaction cell empty yields the highest power.

This design (with the interaction cells empty) yields power gains relative to running two separate experiments, because the control group is used twice. But it avoids the problem of interactions discussed above. An example of such a design is provided by Muralidharan \& Sundararaman (2011) who study the impact of four different interventions in one experiment with one common control group, but no cross-cutting treatment arms.

\footnotetext{
${ }^{24}$ This exact sample split is impossible in any application since $\frac{N}{2}(2-\sqrt{2})$ is not an integer. In our simulations we therefore use $N_{T}^{*}=0.29 \mathrm{~N}$ and $N_{1}^{*}=0.42 \mathrm{~N}$.
} 
Figure 7: Leaving the interaction cell empty increases power for most values of $\beta_{12}$ relative to alternative approaches

(a) Size

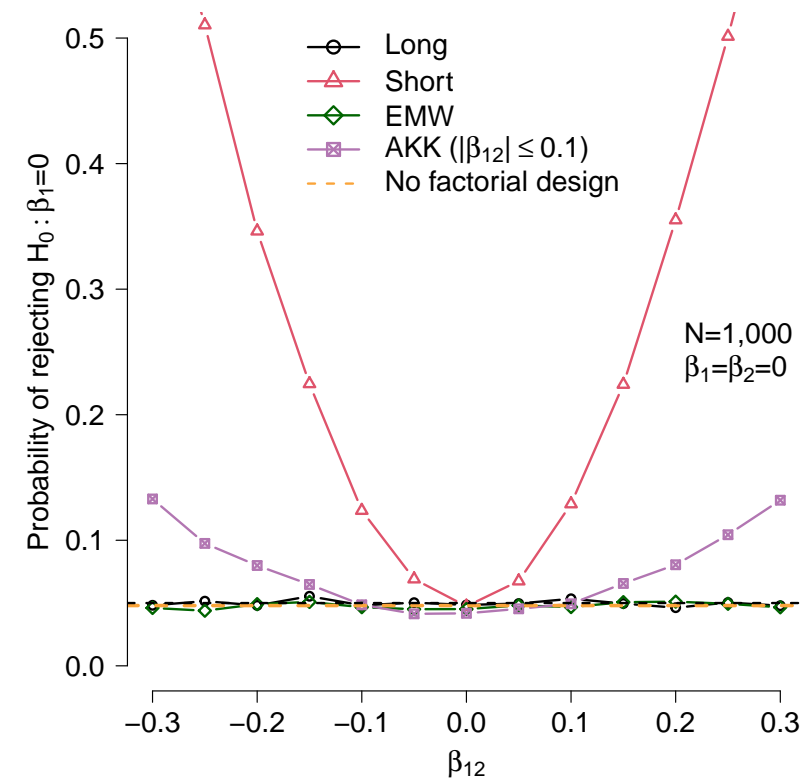

(b) Power

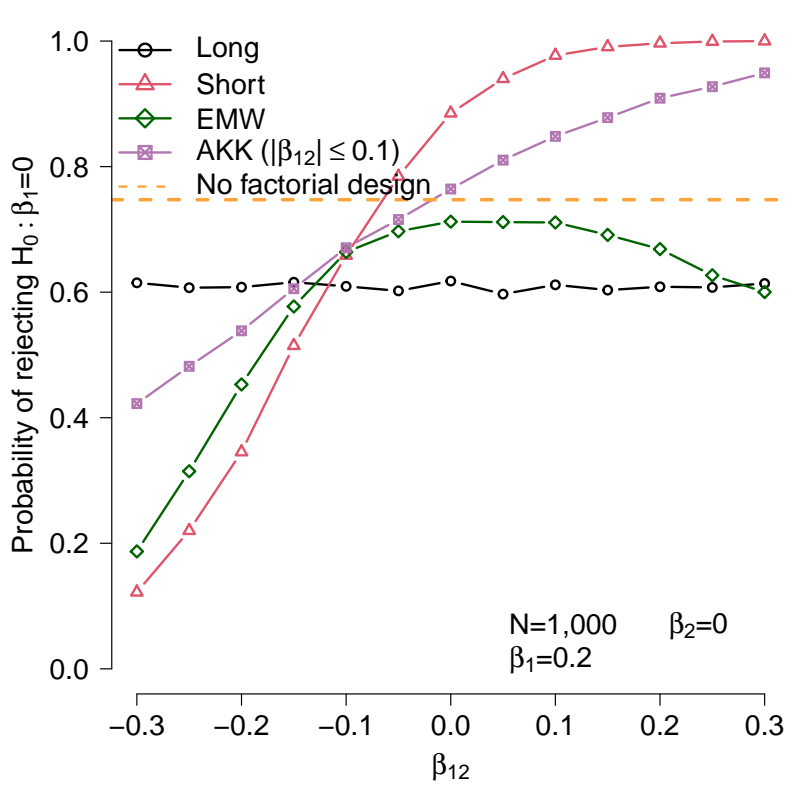

Note: Simulations are based on sample size $N$, normal iid errors, and 10,000 repetitions. The size for figures $7 a$ and $7 b$ is $\alpha=0.05$. EMW refers to Elliott et al. (2015)'s nearly optimal test. AKK refers to Armstrong et al. (2019)'s approach for constructing optimal confidence intervals under prior knowledge about the magnitude of $\beta_{12}$.

\section{Factorial designs with more than two treatments}

So far, our theoretical discussion has focused on $2 \times 2$ factorial designs. Here we briefly discuss designs with more than two treatments.

The theoretical analysis of Section 2 straightforwardly extends to more complicated factorial designs. In particular, estimators based on the long regression model are consistent for the main and interaction effects, whereas the estimators based on the short regression model are consistent for weighted averages of treatment effects with respect to the counterfactuals defined by the other arms of the experiment. The more treatments there are, the more complicated the interpretation of these composite effects will be.

Conceptually, both econometric approaches discussed in Section 5 can be extended beyond $2 \times 2$ settings. However, the nearly optimal tests become computationally prohibitive when there are many interactions (i.e., many nuisance parameters) and, thus, cannot be recommended for complicated factorial designs. Incorporating prior knowledge in the form of restrictions on the magnitude of interactions is computationally fea- 
sible but can be problematic in practice because this approach requires reliable prior knowledge on the magnitude of potentially very many interactions to yield notable power improvements. ${ }^{25}$

Therefore, our recommendation for inference about main effects in more complicated factorial designs is to use $t$-tests based on the long model. These tests are easy to compute irrespective of the dimensionality of the problem and have desirable optimality properties. When the primary parameters of interest are the main effects, we recommend leaving the interaction cells empty at the design stage, which yields power improvements over the $t$-test based on the long model.

\section{Making inferences about interaction effects}

In Sections 4-5, we focused on making inferences on the main effects. Researchers may also use factorial designs to learn about interactions and jointly explore the parameter space of main and interaction effects. We therefore also discuss the use of factorial designs for making inferences on interaction effects.

An important observation is that detecting interaction effects requires much larger sample sizes than detecting main effects. To illustrate, we compare the standard errors of the OLS estimator of the interaction effect, $\hat{\beta}_{12}$, to the standard errors of the OLS estimator of the main effect, $\hat{\beta}_{1}$. Under the assumptions in Section 2.4, the standard errors are

$$
S E\left(\hat{\beta}_{1}\right)=\sigma \sqrt{\frac{N_{1}+N_{2}}{N_{1} N_{2}}} \text { and } S E\left(\hat{\beta}_{12}\right)=\sigma \sqrt{\frac{N_{1} N_{2} N_{3}+N_{1} N_{3} N_{4}+N_{2} N_{3} N_{4}+N_{1} N_{2} N_{4}}{N_{1} N_{2} N_{3} N_{4}}} \text {. }
$$

Note that $S E\left(\hat{\beta}_{1}\right)<S E\left(\hat{\beta}_{12}\right)$, irrespective of the configuration of $\left(N_{1}, N_{2}, N_{3}, N_{4}\right)$. As a consequence, the power for detecting interaction effects is always lower than the power for detecting main effects. Therefore, the required sample size for detecting an interaction effect is always larger than the sample size for detecting a main effect of equal magnitude.

To achieve power $\kappa$, the true interaction effect needs to satisfy (e.g., Duflo et al., 2007; Athey \& Imbens, 2017)

$$
\beta_{12}>\left(\Phi^{-1}(\kappa)+\Phi^{-1}(1-\alpha / 2)\right) S E\left(\hat{\beta}_{12}\right) \equiv M D E_{\beta_{12}} .
$$

\footnotetext{
${ }^{25}$ Both approaches discussed in Appendix A.5 are computationally feasible in more complicated crosscut designs.
} 
Here MDE stands for minimum detectable effect size. Similarly, to achieve power $\kappa$ for detecting the main effect, it must be that

$$
\beta_{1}>\left(\Phi^{-1}(\kappa)+\Phi^{-1}(1-\alpha / 2)\right) S E\left(\hat{\beta}_{1}\right) \equiv M D E_{\beta_{1}} .
$$

We can relate the MDEs to the overall sample size required for detecting interactions, $N_{I}$, and main effects, $N_{M}$, respectively. To illustrate, suppose that the overall sample size is equally distributed across all four cells (which is the optimal design to maximize power for the interaction). In this case, the standard errors are $S E\left(\hat{\beta}_{1}\right)=\sigma \sqrt{8 / N_{M}}$ and $S E\left(\hat{\beta}_{12}\right)=\sigma \sqrt{16 / N_{I}}$, such that

$$
\frac{M D E_{\beta_{1}}}{M D E_{\beta_{12}}}=\frac{\sigma \sqrt{\frac{8}{N_{M}}}}{\sigma \sqrt{\frac{16}{N_{I}}}}
$$

and

$$
2\left(\frac{M D E_{\beta_{1}}}{M D E_{\beta_{12}}}\right)^{2}=\frac{N_{I}}{N_{M}} .
$$

Suppose that the MDE for the interaction effect is half the MDE for the main effect. Then the relative sample size needed to be adequately powered $\left(N_{I} / N_{M}\right)$ is 8 . That is, we need eight times the sample size to detect an interaction effect that is half the size of the main effect. ${ }^{26}$ Even if the MDE for the interaction is the same as the MDE for the main effect, one would need twice the sample size to detect the interaction effect than to detect the main effect. Figure 8a illustrates the general relationship between $N_{I} / N_{M}$ and $M D E_{\beta_{12}} / M D E_{\beta_{1}}$. Given the more onerous sample size requirements to detect interactions relative to main effects, it is not surprising that only few of the interaction effects are significant in the reanalysis in Section 3.2.1.

Further, even when interactions estimates are significant, they can be misleading. This is because significant results in under-powered studies are much more likely to reflect an outlier estimate of the interaction. In particular, as shown by Gelman \& Carlin (2014), low power is associated with a high Type-M error (or exaggeration ratio). The Type-M error is the expectation of the absolute value of the estimator in a hypothetical replication study based on the same design as the original study conditional on being significant,

\footnotetext{
${ }^{26}$ Alternatively, one can compare $M D E_{\beta_{12}}$ to the MDE based on the short model, $M D E_{\beta_{1}^{s}}$, as in Gelman (2018). Because $S E\left(\hat{\beta}_{1}^{s}\right)=\sigma \sqrt{4 / N}$, the required sample size for detecting an interaction is 16 times larger than for detecting main effects based on the short model.
} 
divided by the true effect size (see p.643 and Figure 1 in Gelman \& Carlin, 2014). ${ }^{27}$ For example, if the experiment has $80 \%$ power to detect treatment effects of $0.2 \sigma$ or larger at the $5 \%$ level using the long regression (i.e., a sample of 1,570 equally divided among the four cells) and the true value of the interaction is $0.1 \sigma$, then the Type-M error for $\hat{\beta}_{12}$ is $\sim 251 \%{ }^{28}$ That is, the estimator of the interaction would, on average, be over two times larger than the true value, conditional on being significant. Figure $8 \mathrm{~b}$ shows the general relationship between the Type-M error and $\beta_{12} / M D E_{\beta_{1}}$.

Figure 8: Relative sample size and Type-M error

(a) Relative sample size

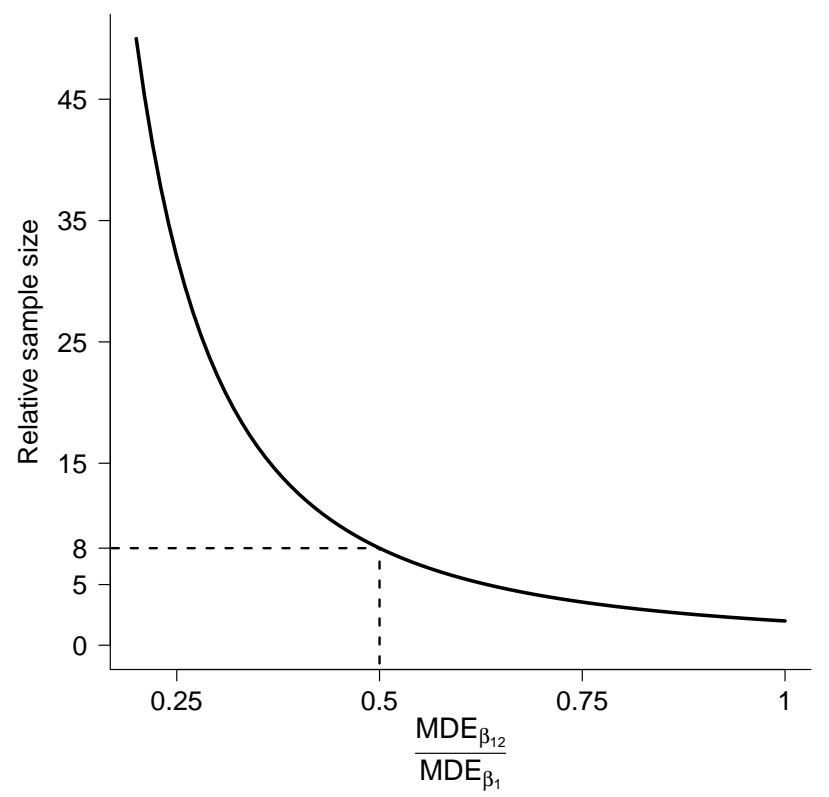

(b) Type-M error

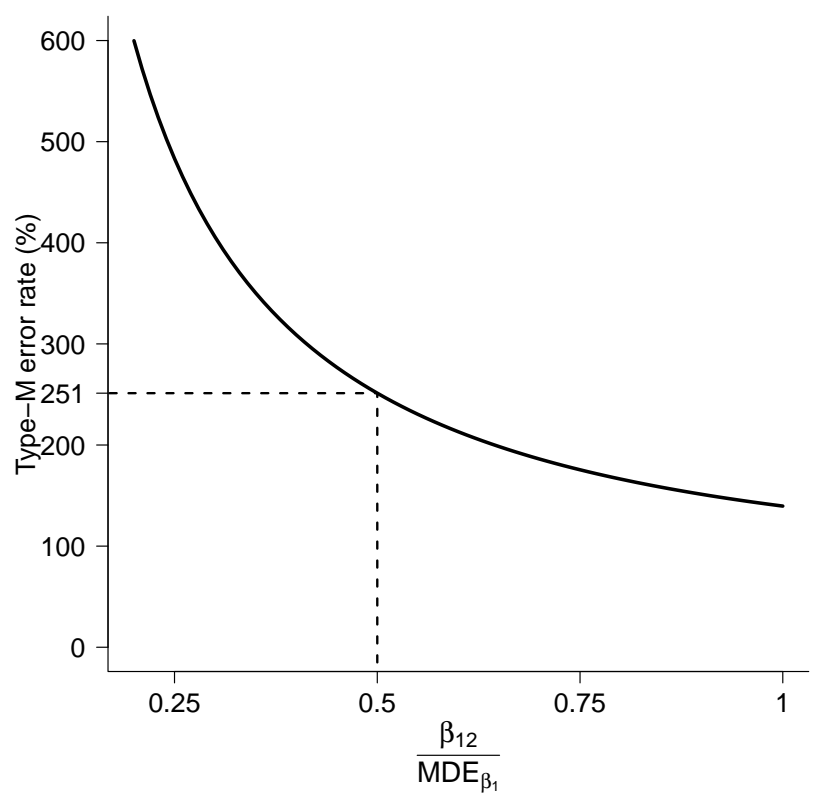

Note: For both panels, we assume the sample is divided equally among the four cells in Table 1. Figure 8a plots the relative sample size $\left(\frac{N_{I}}{N_{M}}\right)$ as a function of the relative $M D E s\left(\frac{M D E_{\beta_{12}}}{M D E_{\beta_{1}}}\right)$. Figure $8 b$ plots the Type-M error for different values of the interaction (relative to the MDE of the main effect, which determines the sample size). We use the closed form formula provided by Lu et al. (2019). We assume that the size is $\alpha=0.05$, that the power is $\kappa=0.8$, and that the MDE for the main effect is 0.2 $\sigma$ (i.e., a sample of 1,570 equally divided among the four cells).

Note that using the long model to estimate and learn about interactions is fine since the OLS estimator of the long model is always unbiased, even if noisy. The problem we document in this section arises because of the focus on statistical significance to assess whether a result is meaningful. Combined with the well-documented publication bias

\footnotetext{
${ }^{27}$ A related problem with under-powered studies is the Type $S$ error rate, which is the probability that conditional on being significant, the estimate of the interaction in a hypothetical replication study based on the same design as the original study has an incorrect sign (see p.643 in Gelman \& Carlin, 2014).

${ }^{28}$ If the experiment has power $80 \%$ to detect treatment effects of $0.2 \sigma$ or larger at the $5 \%$ level using the short regression and the true value of the interaction is $0.1 \sigma$ then the Type-M error for $\hat{\beta}_{12}$ is $\sim 347 \%$.
} 
towards significant results (e.g., Abadie, 2020; I. Andrews \& Kasy, 2018; Christensen \& Miguel, 2018; Franco et al., 2014), the discussion above suggests that published results from under-powered studies are likely to meaningfully exaggerate the true effect. Following Gelman \& Carlin (2014), we suggest studies report power to detect interactions (as well as Type-M errors) in their pre-analysis plan.

\section{Discussion and conclusion}

In this paper we study the theory and practice of inference in factorial designs. We show that the common approaches of directly estimating the short model or doing a twostep model selection procedure yield invalid inferences about the main effects against a "business-as-usual" counterfactual. In contrast, the long model yields consistent estimates, always controls size, and exhibits optimality properties.

We explore if it is possible to increase power to detect main effects in factorial experiments relative to the long model, while controlling size for relevant values of the interaction. The nearly optimal test by Elliott et al. (2015) achieves local power improvements near likely values of the interaction, but can exhibit lower power farther away from such values. Moreover, this approach becomes computationally prohibitive in more complicated factorial designs. Prior knowledge can be explicitly incorporated using the Armstrong et al. (2019) approach. This approach yields power improvements when the prior knowledge is correct, at the cost of size distortions when it is not.

Thus, our recommendation for the analysis of completed experiments is to always present the long regression model. In addition, if researchers would like to focus on results from the short model, they should clearly indicate that treatment effects should be interpreted as a composite effect that includes a weighted-average of interactions with other treatments. It is also important to specify the estimand of interest in advance in a pre-analysis plan, and not justify the short model ex-post based on estimated interactions being insignificant (due to the data-dependent model selection issues we discuss).

For the design of new experiments, if main effects are of primary interest, we recommend leaving the interaction cells empty and increasing the number of units assigned exclusively to the treatment or the control groups. This design-based approach naturally controls size and yields notable global power improvements relative to the long model.

Factorial designs have been motivated by two main considerations: (i) studying more treatments in a cost-effective way, and (ii) learning about interactions. Our discussion and results highlight that both of these uses can be problematic in practice, driven to a large extent by the lack of power to detect interactions. 
This lack of power creates two distinct sets of problems. First, it results in nonrejections of the null of zero interactions (even when they may be meaningful) and leads to researchers incorrectly focusing on the short model assuming that interactions are zero because they are not significant. This leads to a loss of size control and a non-trivial increase in the likelihood of a false rejection of null hypotheses about main effects.

Second, it leads to significant results being much more likely to be over-estimates (Gelman \& Carlin, 2014). This is true for under-powered studies in general, but especially true for interactions because the sample size requirements for having enough power to detect them is much more onerous than to detect main effects. Thus, if interaction effects are of primary interest, we recommend that experiments be explicitly powered to detect interactions and to indicate this in the pre-analysis plan (as in Mbiti et al. (2019)).

Factorial designs do provide an efficient way of learning about multiple treatments as well as their interactions in the same experiment. The problems we highlight stem in large part from using factorial designs in conjunction with a focus on statistical significance for inference on whether treatment effects or interactions are meaningful. This approach reflects the default frequentist paradigm in experimental economics. Going forward, Bayesian methods (that do not privilege a binary "significant or not" threshold for inference) may constitute a promising framework for efficient learning in experiments with cross-cutting designs (e.g., Kassler et al., 2019). 


\section{References}

Abadie, A. (2020, June). Statistical nonsignificance in empirical economics. American Economic Review: Insights, 2(2), 193-208.

Alatas, V., Banerjee, A., Hanna, R., Olken, B. A., \& Tobias, J. (2012, June). Targeting the poor: Evidence from a field experiment in Indonesia. American Economic Review, 102(4), 1206-40. doi: 10.1257/aer.102.4.1206

Allcott, H., \& Taubinsky, D. (2015, August). Evaluating behaviorally motivated policy: Experimental evidence from the lightbulb market. American Economic Review, 105(8), 2501-38. doi: 10.1257/aer.20131564

Andersen, S., Harrison, G. W., Lau, M. I., \& Rutström, E. E. (2008, may). Eliciting risk and time preferences. Econometrica, 76(3), 583-618. doi: 10.1111/j.1468-0262.2008.00848.x

Andreoni, J., Rao, J. M., \& Trachtman, H. (2017). Avoiding the ask: A field experiment on altruism, empathy, and charitable giving. Journal of Political Economy, 125(3), 625-653.

Andrews, D. W. K., \& Guggenberger, P. (2009). Hybrid and size-corrected subsampling methods. Econometrica, 77(3), 721-762.

Andrews, I., \& Kasy, M. (2018). Identification of and correction for publication bias. forthcoming American Economic Review.

Angrist, J. D., \& Krueger, A. B. (1999). Chapter 23 - empirical strategies in labor economics. In O. C. Ashenfelter \& D. Card (Eds.), (Vol. 3, p. 1277 - 1366). Elsevier. doi: https://doi.org/10.1016/S1573-4463(99)03004-7

Angrist, J. D., \& Pischke, J.-S. (2009). Mostly harmless econometrics an empiricist's companion. Princeton University Press.

Armstrong, T. B., \& Kolesar, M. (2018). Optimal inference in a class of regression models. Econometrica, 86(2), 655-683. doi: 10.3982/ECTA14434

Armstrong, T. B., \& Kolesar, M. (2019). Sensitivity analysis using approximate moment condition models. arXiv:1808.07387.

Armstrong, T. B., Kolesar, M., \& Kwon, S. (2019). Optimal inference in regularized regression models. Unpublished Manuscript. 
Ashraf, N., Berry, J., \& Shapiro, J. M. (2010, December). Can higher prices stimulate product use? evidence from a field experiment in zambia. American Economic Review, 100(5), 2383-2413. doi: 10.1257/aer.100.5.2383

Athey, S., \& Imbens, G. W. (2017). The econometrics of randomized experiments. In Handbook of economic field experiments (Vol. 1, pp. 73-140). Elsevier.

Balafoutas, L., Beck, A., Kerschbamer, R., \& Sutter, M. (2013). What drives taxi drivers? a field experiment on fraud in a market for credence goods. Review of Economic Studies, 80(3), 876-891.

Banerjee, A., Cole, S., Duflo, E., \& Linden, L. (2007). Remedying education: Evidence from two randomized experiments in India. The Quarterly Journal of Economics, 122(3), 1235-1264.

Banerjee, A., \& Duflo, E. (2005). Chapter 7 growth theory through the lens of development economics. In P. Aghion \& S. N. Durlauf (Eds.), (Vol. 1, p. 473 - 552). Elsevier.

Bertrand, M., Karlan, D., Mullainathan, S., Shafir, E., \& Zinman, J. (2010). What's advertising content worth? evidence from a consumer credit marketing field experiment. The Quarterly Journal of Economics, 125(1), 263-306. doi: 10.1162/qjec.2010.125.1.263

Blair, G., Cooper, J., Coppock, A., \& Humphreys, M. (2019). Declaring and diagnosing research designs. American Political Science Review, 113(3), 838?859. doi: 10.1017/ S0003055419000194

Blattman, C., Jamison, J. C., \& Sheridan, M. (2017, April). Reducing crime and violence: Experimental evidence from cognitive behavioral therapy in Liberia. American Economic Review, 107(4), 1165-1206. doi: 10.1257/aer.20150503

Brown, J., Hossain, T., \& Morgan, J. (2010). Shrouded attributes and information suppression: Evidence from the field. The Quarterly Journal of Economics, 125(2), 859-876.

Bruhn, M., \& McKenzie, D. (2009, October). In pursuit of balance: Randomization in practice in development field experiments. American Economic Journal: Applied Economics, 1(4), 200-232. doi: 10.1257/app.1.4.200

Carneiro, P., Lee, S., \& Wilhelm, D. (2017). Optimal data collection for randomized control trials. cemmap working paper CWP15/17. 
Christensen, G., \& Miguel, E. (2018, September). Transparency, reproducibility, and the credibility of economics research. Journal of Economic Literature, 56(3), 920-80. doi: $10.1257 /$ jel.20171350

Cochran, W. G., \& Cox, G. M. (1957). Experimental designs. John Wiley \& Sons.

Cohen, J., \& Dupas, P. (2010). Free distribution or cost-sharing? evidence from a randomized malaria prevention experiment. The Quarterly Journal of Economics, 125(1), 1-45. doi: 10.1162/qjec.2010.125.1.1

Cohen, J., Dupas, P., \& Schaner, S. (2015). Price subsidies, diagnostic tests, and targeting of malaria treatment: evidence from a randomized controlled trial. American Economic Review, 105(2), 609-45.

DellaVigna, S., List, J. A., Malmendier, U., \& Rao, G. (2016). Voting to tell others. The Review of Economic Studies, 84(1), 143-181.

Duflo, E., Dupas, P., \& Kremer, M. (2008, July). Peer effects, teacher incentives, and the impact of tracking: Evidence from a randomized evaluation in Kenya. Retrieved from http:// siteresources . worldbank . org/EXTHDOFFICE/Resources/5485726-1239047988859/ 5995659-1239051886394/5996104-1246378480717/Dupas_ETP_07.21.08.pdf (Working paper)

Duflo, E., Dupas, P., \& Kremer, M. (2011). Peer effects, teacher incentives, and the impact of tracking: Evidence from a randomized evaluation in Kenya. American Economic Review, 101(5), 1739-74. doi: 10.1257/aer.101.5.1739

Duflo, E., Dupas, P., \& Kremer, M. (2015a, September). Education, hiv, and early fertility: Experimental evidence from Kenya. American Economic Review, 105(9), 2757-97.

Duflo, E., Dupas, P., \& Kremer, M. (2015b). School governance, teacher incentives, and pupil-teacher experiemental evidence from Kenyan primary schools. Journal of Public Economics, 123, 92-110.

Duflo, E., Glennerster, R., \& Kremer, M. (2007). Using randomization in development economics research: A toolkit. Handbook of development economics, 4, 3895-3962.

Elliott, G., Müller, U. K., \& Watson, M. W. (2015). Nearly optimal tests when a nuisance parameter is present under the null hypothesis. Econometrica, 83(2), 771-811. 
Eriksson, S., \& Rooth, D.-O. (2014, March). Do employers use unemployment as a sorting criterion when hiring? evidence from a field experiment. American Economic Review, 104(3), 1014-39. doi: 10.1257/aer.104.3.1014

Fischer, G. (2013). Contract structure, risk-sharing, and investment choice. Econometrica, 81(3), 883-939.

Fisher, R. A. (1992). The arrangement of field experiments. In S. Kotz \& N. L. Johnson (Eds.), Breakthroughs in statistics: Methodology and distribution (pp. 82-91). New York, NY: Springer New York. Retrieved from https ://doi .org/10.1007/978-1-4612-4380 -9_8 doi: 10.1007/978-1-4612-4380-9_8

Fisman, R., Iyengar, S. S., Kamenica, E., \& Simonson, I. (2008). Racial preferences in dating. The Review of Economic Studies, 75(1), 117-132.

Flory, J. A., Leibbrandt, A., \& List, J. A. (2014). Do competitive workplaces deter female workers? a large-scale natural field experiment on job entry decisions. The Review of Economic Studies, 82(1), 122-155.

Franco, A., Malhotra, N., \& Simonovits, G. (2014). Publication bias in the social sciences: Unlocking the file drawer. Science, 345(6203), 1502-1505. doi: 10.1126/science.1255484

Gelman, A. (2018, Mar). You need 16 times the sample size to estimate an interaction than to estimate a main effect. Retrieved from https://statmodeling.stat.columbia.edu/ 2018/03/15/need-16-times-sample-size-estimate-interaction-estimate-main -effect/

Gelman, A., \& Carlin, J. (2014). Beyond power calculations: Assessing type S (sign) and type M (magnitude) errors. Perspectives on Psychological Science, 9(6), 641-651.

Gerber, A., \& Green, D. (2012). Field experiments: Design, analysis, and interpretation. W. W. Norton.

Gilligan, D. O., Karachiwalla, N., Kasirye, I., Lucas, A., \& Neal, D. (2018, May). Educator Incentives and Educational Triage in Rural Primary Schools (IZA Discussion Papers No. 11516).

Gneezy, U., Leonard, K. L., \& List, J. A. (2009). Gender differences in competition: Evidence from a matrilineal and a patriarchal society. Econometrica, 77(5), 1637-1664. 
Haushofer, J., \& Shapiro, J. (2016). The short-term impact of unconditional cash transfers to the poor: experimental evidence from Kenya. The Quarterly Journal of Economics, 131(4), 1973-2042.

Imbens, G. W., \& Manski, C. F. (2004). Confidence intervals for partially identified parameters. Econometrica, 72(6), 1845-1857. doi: 10.1111/j.1468-0262.2004.00555.x

Jakiela, P., \& Ozier, O. (2015). Does africa need a rotten kin theorem? experimental evidence from village economies. The Review of Economic Studies, 83(1), 231-268.

Joshi, V. M. (1969). Admissibility of the usual confidence sets for the mean of a univariate or bivariate normal population. The Annals of Mathematical Statistics, 40(3), 1042-1067.

Karlan, D., \& List, J. A. (2007, December). Does price matter in charitable giving? evidence from a large-scale natural field experiment. American Economic Review, 97(5), 1774-1793. doi: 10.1257/aer.97.5.1774

Karlan, D., Osei, R., Osei-Akoto, I., \& Udry, C. (2014). Agricultural decisions after relaxing credit and risk constraints. The Quarterly Journal of Economics, 129(2), 597652.

Karlan, D., \& Zinman, J. (2008, June). Credit elasticities in less-developed economies: Implications for microfinance. American Economic Review, 98(3), 1040-68. doi: 10.1257/ aer.98.3.1040

Karlan, D., \& Zinman, J. (2009). Observing unobservables: Identifying information asymmetries with a consumer credit field experiment. Econometrica, 77(6), 1993-2008.

Kassler, D., Nichols-Barrer, I., \& Finucane, M. (2019). Beyond treatment versus control: How bayesian analysis makes factorial experiments feasible in education research. Evaluation Review.

Kaur, S., Kremer, M., \& Mullainathan, S. (2015). Self-control at work. Journal of Political Economy, 123(6), 1227-1277.

Kendall, C., Nannicini, T., \& Trebbi, F. (2015, January). How do voters respond to information? evidence from a randomized campaign. American Economic Review, 105(1), 322-53. doi: 10.1257 /aer.20131063

Kerwin, J. T., \& Thornton, R. L. (2017). Making the grade: The trade-off between efficiency and effectiveness in improving student learning (Working Paper). University of Minnesota. 
Khan, A. Q., Khwaja, A. I., \& Olken, B. A. (2015). Tax farming redux: Experimental evidence on performance pay for tax collectors. The Quarterly Journal of Economics, 131(1), 219-271.

Kleven, H. J., Knudsen, M. B., Kreiner, C. T., Pedersen, S., \& Saez, E. (2011). Unwilling or unable to cheat? evidence from a tax audit experiment in Denmark. Econometrica, 79(3), 651-692. doi: 10.3982/ECTA9113

Kohavi, R., Tang, D., \& Xu, Y. (2020). Trustworthy online controlled experiments: A practical guide to a/b testing. Cambridge University Press. doi: 10.1017/9781108653985

Kremer, M. (2003). Randomized evaluations of educational programs in developing countries: Some lessons. The American Economic Review, 93(2), pp. 102-106.

Leeb, H., \& Pötscher, B. M. (2005). Model selection and inference: Facts and fiction. Econometric Theory, 21(1), 21-59.

Leeb, H., \& Pötscher, B. M. (2006). Can one estimate the conditional distribution of post-model-selection estimators? The Annals of Statistics, 2554-2591.

Leeb, H., \& Pötscher, B. M. (2008). Can one estimate the unconditional distribution of post-model-selection estimators? Econometric Theory, 24(02), 338-376.

Leeb, H., \& Pötscher, B. M. (2017). Testing in the presence of nuisance parameters: Some comments on tests post-model-selection and random critical values. In S. E. Ahmed (Ed.), Big and complex data analysis: Methodologies and applications (pp. 69-82). Cham: Springer International Publishing.

Lehmann, E. L., \& Romano, J. P. (2005). Testing statistical hypotheses. Springer Science \& Business Media.

List, J. A., Sadoff, S., \& Wagner, M. (2011). So you want to run an experiment, now what? some simple rules of thumb for optimal experimental design. Experimental Economics, 14(4), 439.

List, J. A., Shaikh, A. M., \& Xu, Y. (2016). Multiple hypothesis testing in experimental economics. Experimental Economics, 1-21.

Lu, J., Qiu, Y., \& Deng, A. (2019). A note on Type S/M errors in hypothesis testing. British Journal of Mathematical and Statistical Psychology, 72(1), 1-17. 
Mbiti, I., Muralidharan, K., Romero, M., Schipper, Y., Manda, C., \& Rajani, R. (2019, 04). Inputs, incentives, and complementarities in education: Experimental evidence from Tanzania. The Quarterly Journal of Economics, 134(3), 1627-1673. doi: 10.1093/qje/ qjz010

McCloskey, A. (2017). Bonferroni-based size-correction for nonstandard testing problems. Journal of Econometrics.

McCloskey, A. (2019). Asymptotically uniform tests after consistent model selection in the linear regression model. Journal of Business E Economic Statistics, 0(0), 1-35. doi: 10.1080/07350015.2019.1592754

Muralidharan, K., \& Sundararaman, V. (2011). Teacher performance pay: Experimental evidence from India. Journal of Political Economy, 119(1), 39-77.

Olken, B. A. (2007). Monitoring corruption: Evidence from a field experiment in Indonesia. Journal of Political Economy, 115(2), 200-249. doi: 10.1086/517935

Pallais, A., \& Sands, E. G. (2016). Why the referential treatment? evidence from field experiments on referrals. Journal of Political Economy, 124(6), 1793-1828.

Ray, D. (1998). Development economics. Princeton University Press.

Rubin, D. B. (1974). Estimating causal effects of treatments in randomized and nonrandomized studies. Journal of Educational Psychology, 66(5), 688.

Stoye, J. (2009). More on confidence intervals for partially identified parameters. Econometrica, 77(4), 1299-1315. doi: 10.3982/ECTA7347

Thornton, R. L. (2008, December). The demand for, and impact of, learning HIV status. American Economic Review, 98(5), 1829-63. doi: 10.1257/aer.98.5.1829

van der Vaart, A. (1998). Asymptotic statistics. Cambridge University Press.

Young, A. (2018, 11). Channeling Fisher: Randomization Tests and the Statistical Insignificance of Seemingly Significant Experimental Results. The Quarterly Journal of Economics, 134(2), 557-598. doi: 10.1093/qje/qjy029 


\section{A Appendix}

\section{A.1 Papers with factorial designs published in Top-5 economics journals}

Table A.1: Papers with factorial designs published between 2007 and 2017 in top-5 economics journals sorted by citation count (as of July 4, 2019)

\begin{tabular}{|c|c|c|c|c|c|c|c|c|c|}
\hline Authors & Title & Journal & Year & Citations & Treatments & $\begin{array}{l}\text { Interactions } \\
\text { In Design }\end{array}$ & $\begin{array}{l}\text { Interactions } \\
\text { Included }\end{array}$ & $\begin{array}{c}\text { Data } \\
\text { Available }\end{array}$ & $\begin{array}{c}\text { Policy } \\
\text { Evaluation }\end{array}$ \\
\hline Olken (2007) & $\begin{array}{l}\text { Monitoring Corruption: Evi- } \\
\text { dence from a Field Experiment } \\
\text { in Indonesia }\end{array}$ & JPE & 2007 & 1529 & 3 & 2 & 0 & Yes & Yes \\
\hline Banerjee et al. (2007) & $\begin{array}{l}\text { Remedying Education: Evi- } \\
\text { dence from Two Randomized } \\
\text { Experiments in India }\end{array}$ & QJE & 2007 & 1213 & 2 & 1 & 0 & Yes & Yes \\
\hline Duflo et al. (2011) & $\begin{array}{l}\text { Peer Effects, Teacher Incen- } \\
\text { tives, and the Impact of Track- } \\
\text { ing: Evidence from a Random- } \\
\text { ized Evaluation in Kenya }\end{array}$ & AER & 2011 & 787 & 3 & 4 & 0 & Yes & Yes \\
\hline Kleven et al. (2011) & $\begin{array}{l}\text { Unwilling or Unable to Cheat? } \\
\text { Evidence From a Tax Audit Ex- } \\
\text { periment in Denmark }\end{array}$ & ECMA & 2011 & 776 & 2 & 1 & 0 & No & Yes \\
\hline Karlan et al. (2014) & $\begin{array}{l}\text { Agricultural Decisions after } \\
\text { Relaxing Credit and Risk Con- } \\
\text { straints }\end{array}$ & QJE & 2014 & 612 & 2 & 1 & 1 & No & Yes \\
\hline Bertrand et al. (2010) & $\begin{array}{l}\text { What's Advertising Content } \\
\text { Worth? Evidence from a Con- } \\
\text { sumer Credit Marketing Field } \\
\text { Experiment }\end{array}$ & QJE & 2010 & 522 & 14 & 85 & 0 & Yes & No \\
\hline
\end{tabular}




\begin{tabular}{|c|c|c|c|c|c|c|c|c|c|}
\hline \multicolumn{10}{|c|}{ Table A.1 - continued from previous page } \\
\hline Authors & Title & Journal & Year & Citations & Treatments & $\begin{array}{l}\text { Interactions } \\
\text { In Design }\end{array}$ & $\begin{array}{l}\text { Interactions } \\
\text { Included }\end{array}$ & $\begin{array}{c}\text { Data } \\
\text { Available }\end{array}$ & $\begin{array}{c}\text { Policy } \\
\text { Evaluation }\end{array}$ \\
\hline Karlan \& List (2007) & $\begin{array}{l}\text { Does Price Matter in Charita- } \\
\text { ble Giving? Evidence from a } \\
\text { Large-Scale Natural Field Ex- } \\
\text { periment }\end{array}$ & AER & 2007 & 506 & 7 & 28 & 0 & Yes & No \\
\hline Thornton (2008) & $\begin{array}{l}\text { The Demand for, and Impact } \\
\text { of, Learning HIV Status }\end{array}$ & AER & 2008 & 453 & 2 & 1 & 0 & Yes & Yes \\
\hline $\begin{array}{l}\text { Haushofer \& Shapiro } \\
\text { (2016) }\end{array}$ & $\begin{array}{l}\text { The Short-term Impact of Un- } \\
\text { conditional Cash Transfers to } \\
\text { the Poor: Experimental Evi- } \\
\text { dence from Kenya }\end{array}$ & QJE & 2016 & 393 & 6 & 8 & 3 & Yes & Yes \\
\hline Alatas et al. (2012) & $\begin{array}{l}\text { Targeting the Poor: Evidence } \\
\text { from a Field Experiment in In- } \\
\text { donesia }\end{array}$ & AER & 2012 & 330 & 4 & 16 & 0 & Yes & Yes \\
\hline Karlan \& Zinman (2008) & $\begin{array}{l}\text { Credit Elasticities in Less- } \\
\text { Developed Economies: Impli- } \\
\text { cations for Microfinance }\end{array}$ & AER & 2008 & 311 & 3 & 2 & 0 & Yes & No \\
\hline Duflo et al. (2015a) & $\begin{array}{l}\text { Education, HIV, and Early Fer- } \\
\text { tility: Experimental Evidence } \\
\text { from Kenya }\end{array}$ & AER & 2015 & 282 & 3 & 3 & 1 & Yes & Yes \\
\hline Andreoni et al. (2017) & $\begin{array}{l}\text { Avoiding the Ask: A Field Ex- } \\
\text { periment on Altruism, Empa- } \\
\text { thy, and Charitable Giving }\end{array}$ & JPE & 2017 & 270 & 2 & 1 & 1 & Yes & No \\
\hline Jakiela \& Ozier (2015) & $\begin{array}{l}\text { Does Africa Need a Rotten Kin } \\
\text { Theorem? Experimental Evi- } \\
\text { dence from Village Economies }\end{array}$ & ReStud & 2016 & 245 & 3 & 6 & 6 & Yes & No \\
\hline
\end{tabular}




\begin{tabular}{|c|c|c|c|c|c|c|c|c|c|}
\hline \multicolumn{10}{|c|}{ Table A.1 - continued from previous page } \\
\hline Authors & Title & Journal & Year & Citations & Treatments & $\begin{array}{l}\text { Interactions } \\
\text { In Design }\end{array}$ & $\begin{array}{l}\text { Interactions } \\
\text { Included }\end{array}$ & $\begin{array}{c}\text { Data } \\
\text { Available }\end{array}$ & $\begin{array}{c}\text { Policy } \\
\text { Evaluation }\end{array}$ \\
\hline Eriksson \& Rooth (2014) & $\begin{array}{l}\text { Do Employers Use Unemploy- } \\
\text { ment as a Sorting Criterion } \\
\text { When Hiring? Evidence from } \\
\text { a Field Experiment }\end{array}$ & AER & 2014 & 238 & 34 & 71680 & 0 & Yes & No \\
\hline $\begin{array}{l}\text { Allcott \& } \quad \text { Taubinsky } \\
\text { (2015) }\end{array}$ & $\begin{array}{l}\text { Evaluating Behaviorally Mo- } \\
\text { tivated Policy: Experimental } \\
\text { Evidence from the Lightbulb } \\
\text { Market }\end{array}$ & AER & 2015 & 237 & 2 & 1 & 0 & No & No \\
\hline Flory et al. (2014) & $\begin{array}{l}\text { Do Competitive Workplaces } \\
\text { Deter Female Workers? A } \\
\text { Large-Scale Natural Field Ex- } \\
\text { periment on Job Entry Deci- } \\
\text { sions }\end{array}$ & ReStud & 2015 & 204 & 10 & 24 & 12 & Yes & No \\
\hline Brown et al. (2010) & $\begin{array}{l}\text { Shrouded Attributes and In- } \\
\text { formation Suppression: Evi- } \\
\text { dence from the Field }\end{array}$ & QJE & 2010 & 189 & 3 & 6 & 6 & No & No \\
\hline DellaVigna et al. (2016) & Voting to Tell Others & ReStud & 2017 & 169 & 4 & 15 & 0 & Yes & No \\
\hline Fischer (2013) & $\begin{array}{l}\text { Contract Structure, Risk- } \\
\text { Sharing, and Investment } \\
\text { Choice }\end{array}$ & ECMA & 2013 & 162 & 7 & 9 & 9 & Yes & No \\
\hline Kaur et al. (2015) & Self-Control at Work & JPE & 2015 & 154 & 8 & 16 & 0 & Yes & No \\
\hline Cohen et al. (2015) & $\begin{array}{l}\text { Price Subsidies, Diagnostic } \\
\text { Tests, and Targeting of Malaria } \\
\text { Treatment: Evidence from a } \\
\text { Randomized Controlled Trial }\end{array}$ & AER & 2015 & 151 & 3 & 7 & 7 & Yes & Yes \\
\hline
\end{tabular}




\begin{tabular}{|c|c|c|c|c|c|c|c|c|c|}
\hline \multicolumn{10}{|c|}{ Table A.1 - continued from previous page } \\
\hline Authors & Title & Journal & Year & Citations & Treatments & $\begin{array}{l}\text { Interactions } \\
\text { In Design }\end{array}$ & $\begin{array}{l}\text { Interactions } \\
\text { Included }\end{array}$ & $\begin{array}{c}\text { Data } \\
\text { Available }\end{array}$ & $\begin{array}{l}\text { Policy } \\
\text { Evaluation }\end{array}$ \\
\hline Blattman et al. (2017) & $\begin{array}{l}\text { Reducing Crime and Violence: } \\
\text { Experimental Evidence from } \\
\text { Cognitive Behavioral Therapy } \\
\text { in Liberia }\end{array}$ & AER & 2017 & 135 & 2 & 1 & 1 & Yes & Yes \\
\hline Khan et al. (2015) & $\begin{array}{l}\text { Tax Farming Redux: Exper- } \\
\text { imental Evidence on Perfor- } \\
\text { mance Pay for Tax Collectors }\end{array}$ & QJE & 2016 & 133 & 6 & 8 & 0 & Yes & Yes \\
\hline Balafoutas et al. (2013) & $\begin{array}{l}\text { What Drives Taxi Drivers? A } \\
\text { Field Experiment on Fraud in } \\
\text { a Market for Credence Goods }\end{array}$ & ReStud & 2013 & 126 & 5 & 6 & 0 & Yes & No \\
\hline Kendall et al. (2015) & $\begin{array}{l}\text { How Do Voters Respond to In- } \\
\text { formation? Evidence from a } \\
\text { Randomized Campaign }\end{array}$ & AER & 2015 & 116 & 5 & 5 & 5 & Yes & No \\
\hline Pallais \& Sands (2016) & $\begin{array}{l}\text { Why the Referential Treat- } \\
\text { ment? Evidence from Field Ex- } \\
\text { periments on Referrals }\end{array}$ & JPE & 2016 & 85 & 3 & 12 & 0 & No & No \\
\hline
\end{tabular}

Note: This table provides relevant information from all articles with factorial designs published in top-5 journals. Citation counts are from Google Scholar on July 4th of 2019. Treatments is the number of different treatments in the paper. "Interactions in Design" is the number of interactions in the experimental design. "Interactions Included" is the number of interactions included in the main specification of the paper. Data available, refers to whether the data is publicly available or not. Allcott \& Taubinsky (2015) has two field experiments. The table refers to the second one. Section B.1.16 provides for more details. One of the three dimensions of randomization in Flory et al. (2014) does not appear in the publicly available data. Online Appendix B.1 (in http: //mauricio-romero .com/pdfs/papers/Appendix_crosscuts .pdf) describes the experimental design of each of the 27 papers. 


\section{A.1.1 All papers}

Figure A.1: Distribution of the $t$-value of interaction terms across studies

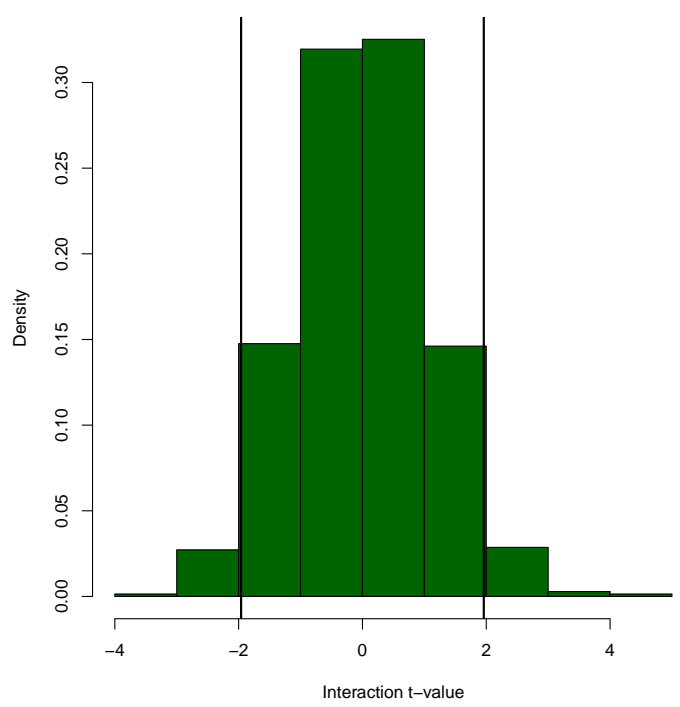

Note: If studies have factorial designs that cross-randomize more than two treatments only two-way interactions are included in this calculation. The vertical lines are at \pm 1.96 . 


\section{A.1.2 Ten most cited papers}

Figure A.2: Treatment estimates based on the long and the short model

(a) Main treatment estimates

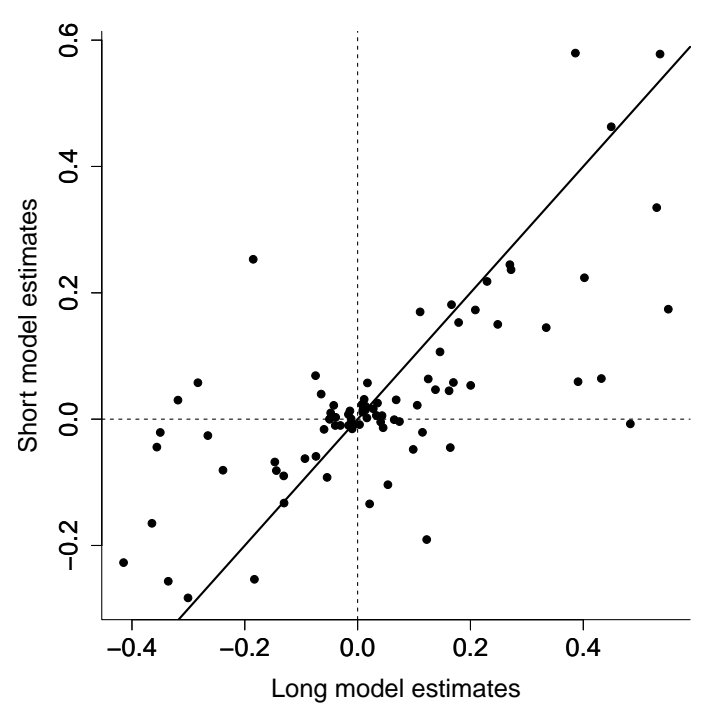

(b) Interaction

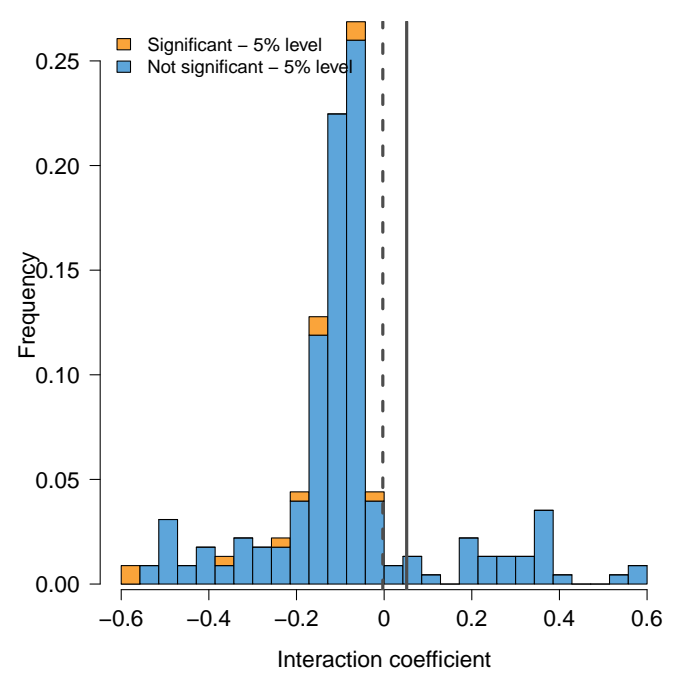

Note: Both figures show treatment estimates from the ten most cited papers with factorial designs and publicly available data that do not include the interactions in the original study. Figure A.2a shows how the main treatment estimates change across the short and the long model across studies. The median main treatment estimate from the short model is $0.01 \sigma$, the median main treatment estimate from the long model is $0.01 \sigma$, the average absolute difference between the treatment estimates of the short and the long model is $0.05 \sigma$, the median absolute difference in percentage terms between the treatment estimates of the short and the long model is $131 \%$, and $28 \%$ of treatment estimates change sign when they are estimated using the long instead of the short model. Figure A.2b shows the distribution of the interactions between the main treatments. We trim the top and bottom $1 \%$ of the distribution. The median interaction is $-0.00 \sigma$ (dashed vertical line), the median absolute value of the interactions is $0.05 \sigma$ (dashed vertical line), $5.6 \%$ of interactions are significant at the $10 \%$ level, $2.6 \%$ are significant at the $5 \%$ level, and $0.0 \%$ are significant at the $1 \%$ level, and the median relative absolute value of the interaction with respect to the main treatment effect is 0.37 . 
Table A.2: Significance of treatment estimates based on the long and the short model

\begin{tabular}{|c|c|c|c|}
\hline \multicolumn{3}{|c|}{ Panel A: Significance at the $10 \%$ level } & \multirow[b]{2}{*}{ Total } \\
\hline With interaction & Not significant & Significant & \\
\hline Not significant & 49 & 13 & 62 \\
\hline Significant & 6 & 17 & 23 \\
\hline Total & 55 & 30 & 85 \\
\hline \multicolumn{4}{|c|}{ Panel B: Significance at the $5 \%$ level } \\
\hline With interaction & Not significant & Significant & Total \\
\hline Not significant & 60 & 9 & 69 \\
\hline Significant & 4 & 12 & 16 \\
\hline Total & 64 & 21 & 85 \\
\hline \multicolumn{4}{|c|}{$\begin{array}{r}\text { Panel C: Significance at the } 1 \% \text { level } \\
\text { Without interaction }\end{array}$} \\
\hline With interaction & Not significant & Significant & Total \\
\hline Not significant & 73 & 3 & 76 \\
\hline Significant & 1 & 8 & 9 \\
\hline Total & 74 & 11 & 85 \\
\hline
\end{tabular}

This table shows the number of coefficients that are significant at a given level when estimating the long regression (columns) and the short regression (rows). This table only includes information from the ten most cited papers with factorial designs and publicly available data that do not include the interactions in the original study. Table 3 has data for all papers with factorial designs and publicly available data that do not include the interaction in the original study. Panel A uses a 10\% significance level, Panel B uses 5\%, and Panel C uses 1\%. 
Figure A.3: Distribution of the $t$-value of interaction terms across studies

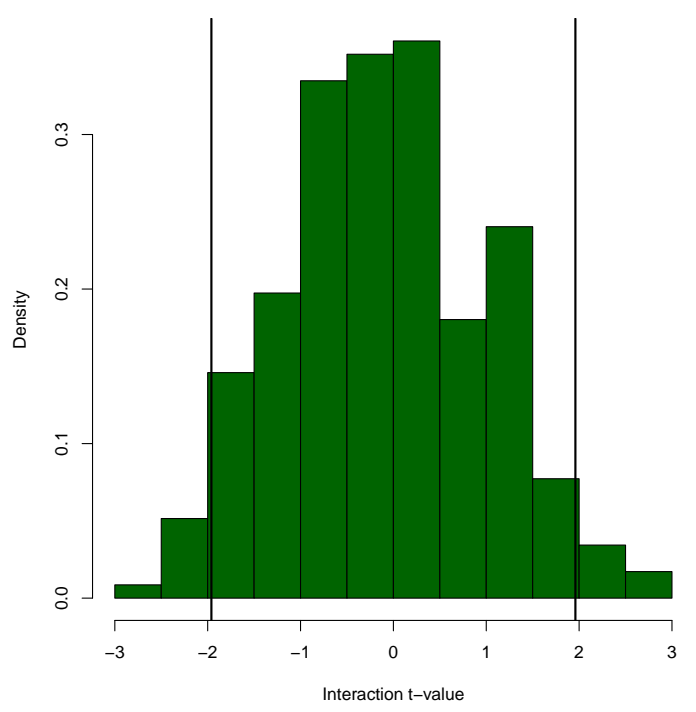

Note: If studies have factorial designs that cross-randomize more than two treatments only two-way interactions are included in this calculation. The vertical lines are at \pm 1.96 . 


\section{A.1.3 Policy experiments}

Figure A.4: Treatment estimates from the long and the short regression

(a) Main treatment estimates

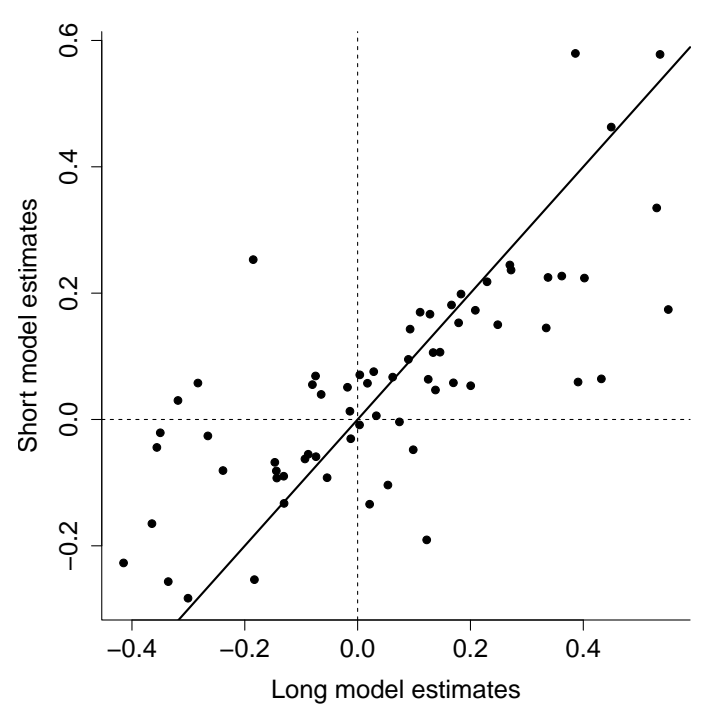

(b) Interaction

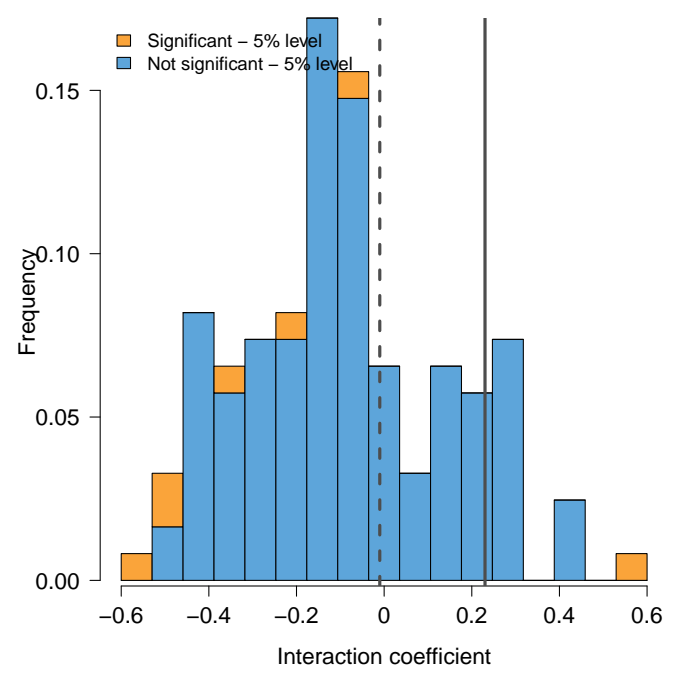

Note: Both figures show treatment estimates from the papers with factorial designs and publicly available data that do not include the interactions in the original study and do policy evaluation. Figure A.4a shows how the main treatment estimates change across the short and the long model across studies. The median main treatment estimate from the short model is $0.06 \sigma$, the median main treatment estimate from the long model is $0.05 \sigma$, the average absolute difference between the treatment estimates of the short and the long model is $0.07 \sigma$, the median absolute difference in percentage terms between the treatment estimates of the short and the long model is 69\%, and $21 \%$ of treatment estimates change sign when they are estimated using the long model instead of the short model. Figure A.4b shows the distribution of the interactions between the main treatments. We trim the top and bottom $1 \%$ of the distribution. The median interaction is $-0.01 \sigma$ (dashed vertical line), the median absolute value of interactions is $0.23 \sigma$ (solid vertical line), $6.3 \%$ of interactions are significant at the $10 \%$ level, $3.2 \%$ are significant at the $5 \%$ level, and $0.0 \%$ are significant at the $1 \%$ level, and the median relative absolute value of the interaction with respect to the main treatment effect is 1.01 . 
Table A.3: Significance of treatment estimates from the long and the short regression

\begin{tabular}{|c|c|c|c|}
\hline \multicolumn{3}{|c|}{ Panel A: Significance at the $10 \%$ level } & \multirow[b]{2}{*}{ Total } \\
\hline With interaction & Not significant & Significant & \\
\hline Not significant & 31 & 10 & 41 \\
\hline Significant & 5 & 21 & 26 \\
\hline Total & 36 & 31 & 67 \\
\hline \multicolumn{4}{|c|}{ Panel B: Significance at the $5 \%$ level } \\
\hline With interaction & Not significant & Significant & Total \\
\hline Not significant & 43 & 6 & 49 \\
\hline Significant & 5 & 13 & 18 \\
\hline Total & 48 & 19 & 67 \\
\hline \multicolumn{4}{|c|}{$\begin{array}{r}\text { Panel C: Significance at the } 1 \% \text { level } \\
\text { Without interaction }\end{array}$} \\
\hline With interaction & Not significant & Significant & Total \\
\hline Not significant & 56 & 3 & 59 \\
\hline Significant & 1 & 7 & 8 \\
\hline Total & 57 & 10 & 67 \\
\hline
\end{tabular}

This table shows the number of coefficients that are significant at a given level when estimating the long regression (columns) and the short regression (rows). This table only includes information from papers with factorial designs and publicly available data that do not include the interactions in the original study and do policy evaluation. Table 3 has data for all papers with factorial designs and publicly available data that do not include the interaction in the original study. Panel A uses a $10 \%$ significance level, Panel B uses 5\%, and Panel C uses 1\%. 
Figure A.5: Distribution of the $t$-value of interaction terms across studies

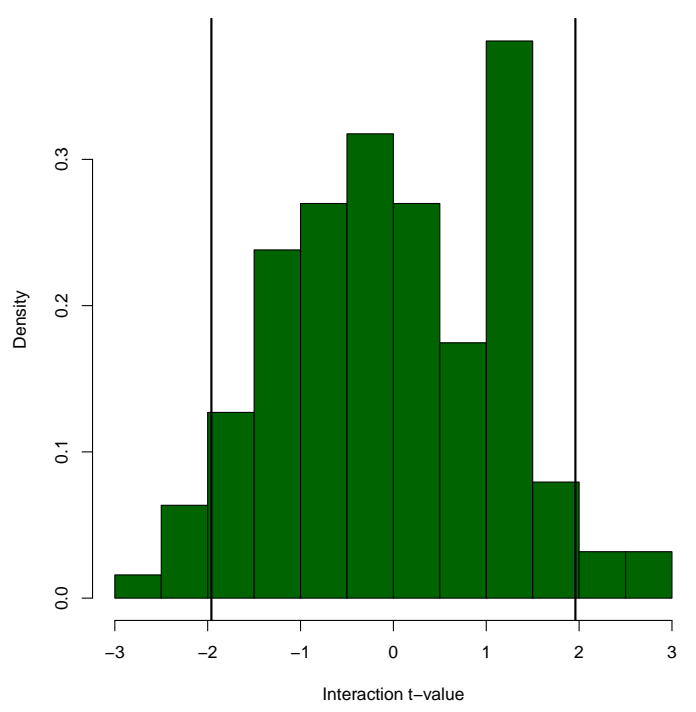

Note: If studies have factorial designs that cross-randomize more than two treatments only two-way interactions are included in this calculation. The vertical lines are at \pm 1.96 . 


\section{A.1.4 Studies with all interactions included}

Figure A.6: Treatment estimates based on the long and the short model

(a) Main treatment estimates

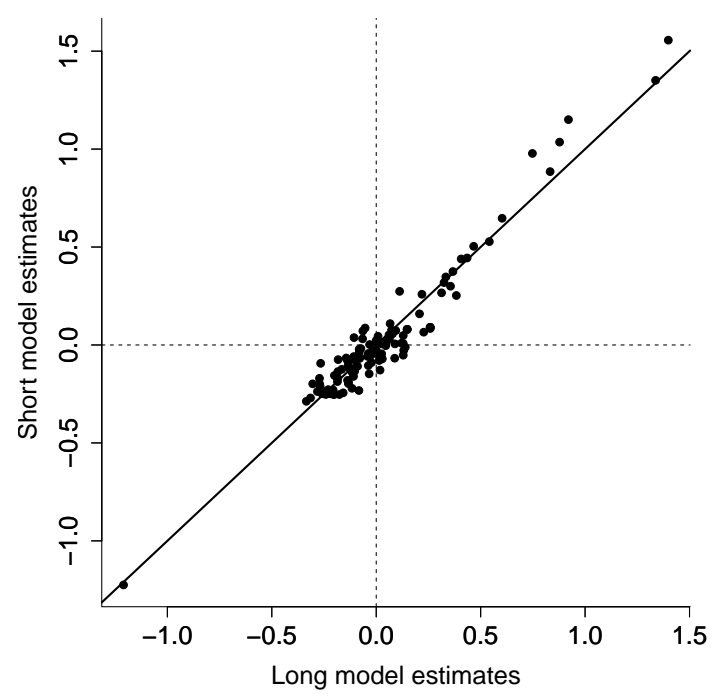

(b) Interaction

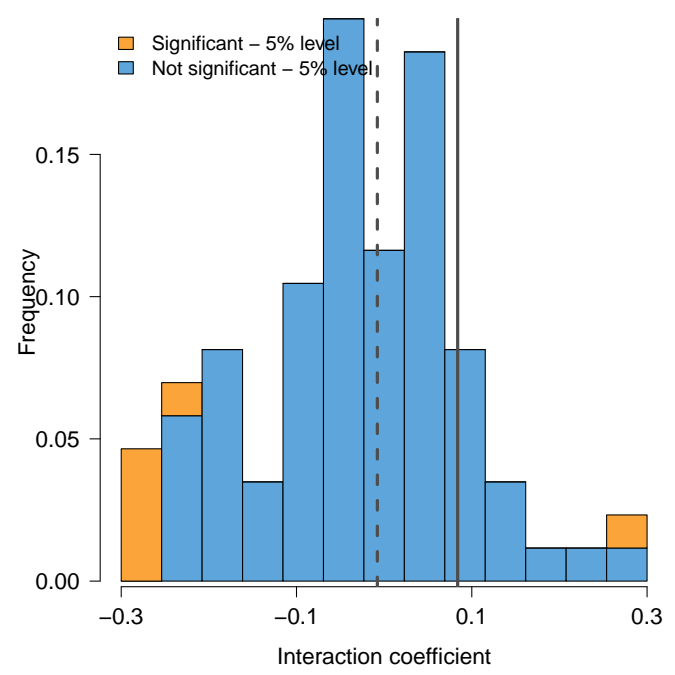

Note: Both figures show treatment estimates from the papers with factorial designs and publicly available data that do not include the interaction in the original study and do policy evaluation. Figure A.6a shows how the main treatment estimates change across the short and the long model across studies. The median main treatment estimate from the short model is $-0.03 \sigma$, the median main treatment estimate from the long model is $-0.02 \sigma$, the average absolute difference between the treatment estimates of the short and the long model is $0.05 \sigma$, the median absolute difference in percentage terms between the treatment estimates of the short and the long model is 37\%, and $15 \%$ of treatment estimates change sign when they are estimated using the long or the short model. Figure A.6b shows the distribution of the interactions between the main treatments. We trim the top and bottom $1 \%$ of the distribution. The median interaction is $-0.01 \sigma$ (dashed vertical line), the median absolute value of interactions is $0.08 \sigma$ (solid vertical line), $4.5 \%$ of interactions are significant at the $10 \%$ level, $1.1 \%$ are significant at the $5 \%$ level, and $0.0 \%$ are significant at the $1 \%$ level, and the median relative absolute value of the interaction with respect to the main treatment effect is 0.52 . 
Table A.4: Significance of treatment estimates based on the long and the short model

\begin{tabular}{|c|c|c|c|}
\hline \multicolumn{3}{|c|}{ Panel A: Significance at the $10 \%$ level } & \multirow[b]{2}{*}{ Total } \\
\hline With interaction & Not significant & Significant & \\
\hline Not significant & 61 & 13 & 74 \\
\hline Significant & 4 & 39 & 43 \\
\hline Total & 65 & 52 & 117 \\
\hline \multicolumn{4}{|c|}{ Panel B: Significance at the $5 \%$ level } \\
\hline With interaction & Not significant & Significant & Total \\
\hline Not significant & 68 & 10 & 78 \\
\hline Significant & 6 & 33 & 39 \\
\hline Total & 74 & 43 & 117 \\
\hline \multicolumn{4}{|c|}{ Panel C: Significance at the $1 \%$ level } \\
\hline With interaction & Not significant & Significant & Total \\
\hline Not significant & 77 & 12 & 89 \\
\hline Significant & 2 & 26 & 28 \\
\hline Total & 79 & 38 & 117 \\
\hline
\end{tabular}

This table shows the number of coefficients that are significant at a given level when estimating the long regression (columns) and the short regression (rows). This table only includes information from papers with factorial designs and publicly available data that do include the interaction in the original study. Table 3 has data for all papers with factorial designs and publicly available data that do not include the interaction in the original study. Panel A uses a 10\% significance level, Panel B uses 5\%, and Panel C uses 1\%. 
Figure A.7: Distribution of the $t$-value of interaction terms across studies

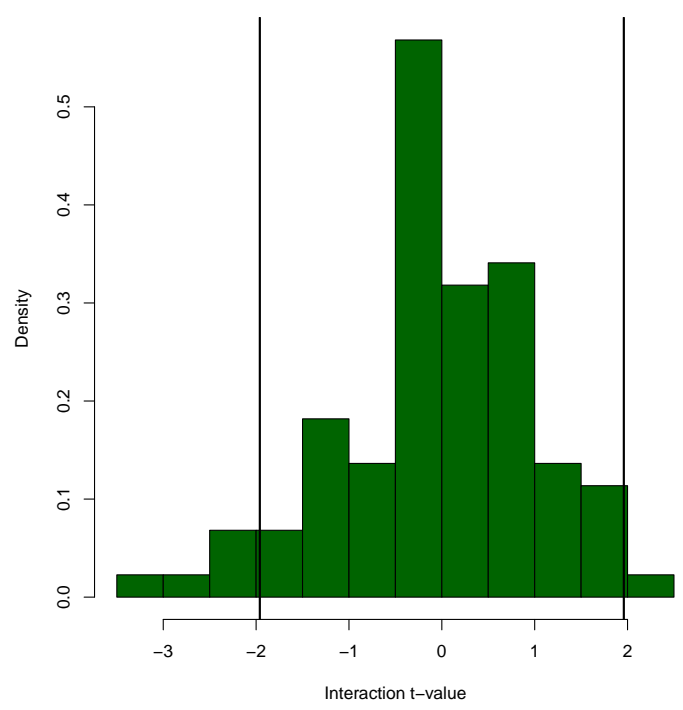

Note: If studies have factorial designs that cross-randomize more than two treatments only two-way interactions are included in this calculation. The vertical lines are at \pm 1.96 .

\section{A.2 Derivation of expressions for the regression coefficients}

\section{A.2.1 Derivation of the expressions for $\beta_{1}, \beta_{2}$, and $\beta_{12}$}

Because the long regression model (3) is fully saturated, we have

$$
\begin{aligned}
\beta_{1}= & E\left(Y \mid T_{1}=1, T_{2}=0\right)-E\left(Y \mid T_{1}=0, T_{2}=0\right), \\
\beta_{2}= & E\left(Y \mid T_{1}=0, T_{2}=1\right)-E\left(Y \mid T_{1}=0, T_{2}=0\right), \\
\beta_{12}= & E\left(Y \mid T_{1}=1, T_{2}=1\right)-E\left(Y \mid T_{1}=0, T_{2}=1\right) \\
& -\left[E\left(Y \mid T_{1}=1, T_{2}=0\right)-E\left(Y \mid T_{1}=0, T_{2}=0\right)\right] .
\end{aligned}
$$

Random assignment and the definition of potential outcomes in Equation (1) imply that, for $\left(t_{1}, t_{2}\right) \in\{0,1\} \times\{0,1\}$,

$$
\begin{aligned}
E\left(Y \mid T_{1}=t_{1}, T_{2}=t_{2}\right) & =E\left(Y_{t_{1}, t_{2}} \mid T_{1}=t_{1}, T_{2}=t_{2}\right) \\
& =E\left(Y_{t_{1}, t_{2}}\right) .
\end{aligned}
$$


Thus, it follows that

$$
\begin{aligned}
\beta_{1} & =E\left(Y_{1,0}-Y_{0,0}\right), \\
\beta_{2} & =E\left(Y_{0,1}-Y_{0,0}\right), \\
\beta_{12} & =E\left(Y_{1,1}-Y_{0,1}-Y_{1,0}+Y_{0,0}\right) .
\end{aligned}
$$

\section{A.2.2 Derivation of the expressions for $\beta_{1}^{s}$ and $\beta_{2}^{s}$}

Here we derive (8). Equation (9) then follows from rearranging terms. The derivations of Equations (10) and (11) are similar and thus omitted.

For the short regression model (4), independence of $T_{1}$ and $T_{2}$ implies that

$$
\beta_{1}^{S}=E\left(Y \mid T_{1}=1\right)-E\left(Y \mid T_{1}=0\right) .
$$

Consider

$$
\begin{aligned}
E\left(Y \mid T_{1}=1\right)= & E\left(Y \mid T_{1}=1, T_{2}=1\right) P\left(T_{2}=1 \mid T_{1}=1\right) \\
& +E\left(Y \mid T_{1}=1, T_{2}=0\right) P\left(T_{2}=0 \mid T_{1}=1\right) \\
= & E\left(Y_{1,1}\right) P\left(T_{2}=1\right)+E\left(Y_{1,0}\right) P\left(T_{2}=0\right),
\end{aligned}
$$

where the first equality follows from the law of iterated expectations and the second equality follows by the definition of potential outcomes and random assignment. Similarly, obtain

$$
E\left(Y \mid T_{1}=0\right)=E\left(Y_{0,1}\right) P\left(T_{2}=1\right)+E\left(Y_{0,0}\right) P\left(T_{2}=0\right) .
$$

Thus, we have

$$
\begin{aligned}
\beta_{1}^{S} & =E\left(Y \mid T_{1}=1\right)-E\left(Y \mid T_{1}=0\right) \\
& =E\left(Y_{1,1}-Y_{0,1}\right) P\left(T_{2}=1\right)+E\left(Y_{1,0}-Y_{0,0}\right) P\left(T_{2}=0\right) .
\end{aligned}
$$

\section{A.2.3 Consistency of the OLS estimators based on model (18)}

Here we show that when the interaction cell is empty and $T_{1}$ and $T_{2}$ are randomly assigned, the OLS estimators based on the regression model (18) are consistent for the main effects. 
Define $\hat{\beta}^{*} \equiv\left(\hat{\beta}_{0}^{*}, \hat{\beta}_{1}^{*}, \hat{\beta}_{2}^{*}\right)^{\prime}$ and $\beta^{*} \equiv\left(\beta_{0,}^{*}, \beta_{1}^{*}, \beta_{2}^{*}\right)^{\prime}=E\left(X X^{\prime}\right)^{-1} E(X Y)$, where $X=$ $\left(1, T_{1}, T_{2}\right)^{\prime}$. Under standard conditions, $\hat{\beta}^{*} \stackrel{p}{\rightarrow} \beta^{*}$. Hence, it remains to show that $\beta_{1}^{*}$ and $\beta_{2}^{*}$ are equal to the main effects. In what follows, we focus on $\beta_{1}^{*}$; the derivation for $\beta_{2}^{*}$ is similar. To simplify the exposition, we define $p_{1} \equiv P\left(T_{1}=1\right), p_{2} \equiv P\left(T_{2}=1\right)$ and $p_{12} \equiv P\left(T_{1}=1, T_{2}=1\right)$.

Multiplying out yields the following expressions for $\beta_{1}^{*}$ :

$$
\beta_{1}^{*}=\frac{\left(p_{2} p_{12}-p_{1} p_{2}\right) E(Y)+p_{1}\left(p_{2}-p_{2}^{2}\right) E\left(Y \mid T_{1}=1\right)+p_{2}\left(p_{1} p_{2}-p_{12}\right) E\left(Y \mid T_{2}=1\right)}{-p_{1}^{2} p_{2}-p_{1} p_{2}^{2}+p_{1} p_{2}+2 p_{1} p_{2} p_{12}-p_{12}^{2}} .
$$

Using the fact that the interaction cell is empty, which implies that $p_{12}=0$, obtain

$$
\beta_{1}^{*}=\frac{-p_{1} p_{2} E(Y)+p_{1} p_{2}\left(1-p_{2}\right) E\left(Y \mid T_{1}=1\right)+p_{1} p_{2}^{2} E\left(Y \mid T_{2}=1\right)}{-p_{1}^{2} p_{2}-p_{1} p_{2}^{2}+p_{1} p_{2}}
$$

Because $p_{12}=0$, we have that

$E(Y)=E\left(Y \mid T_{1}=1, T_{2}=0\right) p_{1}+E\left(Y \mid T_{1}=0, T_{2}=0\right)\left(1-p_{1}-p_{2}\right)+E\left(Y \mid T_{1}=0, T_{2}=1\right) p_{2}$.

Combining (19) and (20) and simplifying yields:

$$
\beta_{1}^{*}=E\left(Y \mid T_{1}=1, T_{2}=0\right)-E\left(Y \mid T_{1}=0, T_{2}=0\right)
$$

The result now follows by random assignment of $T_{1}$ and $T_{2}$ and the definition of potential outcomes.

\section{A.3 Variance reductions and power gains based on the short model}

\section{A.3.1 Formal power comparison between the short and the long model}

Suppose that the researcher has access to a random sample $\left\{Y_{i}, T_{1 i}, T_{2 i}\right\}_{i=1}^{N}$ and that the data are generated according to the following linear model

$$
Y_{i}=\beta_{0}+\beta_{1} T_{1 i}+\beta_{2} T_{2 i}+\beta_{12} T_{1 i} T_{2 i}+\varepsilon_{i}
$$

where $\varepsilon_{i} \sim N\left(0, \sigma^{2}\right)$ is independent of $\left(T_{1 i}, T_{2 i}\right)$ and $\sigma^{2}$ is known. Normality allows us to compute the finite sample power and to formally compare the $t$-tests based on the long and the short regression model. In what follows, we focus on $\beta_{1}$. The analysis for $\beta_{2}$ is symmetric and omitted. 
Define $\boldsymbol{T}_{1} \equiv\left(T_{11}, \ldots, T_{1 N}\right)^{\prime}$ and $\boldsymbol{T}_{2} \equiv\left(T_{21}, \ldots, T_{2 N}\right)^{\prime}$. If the interaction effect is zero (i.e., $\left.\beta_{12}=0\right)$, it follows from standard results that, conditional on $\left(\boldsymbol{T}_{1}, \boldsymbol{T}_{2}\right), \hat{\beta}_{1} \sim$ $N\left(\beta_{1}, \operatorname{Var}\left(\hat{\beta}_{1}\right)\right)$ and $\hat{\beta}_{1}^{s} \sim N\left(\beta_{1}, \operatorname{Var}\left(\hat{\beta}_{1}^{s}\right)\right)$, where

$\operatorname{Var}\left(\hat{\beta}_{1}\right)=\sigma^{2} \frac{N_{1}+N_{2}}{N_{1} N_{2}}$ and $\operatorname{Var}\left(\hat{\beta}_{1}^{s}\right)=\sigma^{2} \frac{N_{1} N_{3}+N_{1} N_{4}+N_{2} N_{3}+N_{2} N_{4}}{N_{1} N_{2} N_{3}+N_{1} N_{2} N_{4}+N_{1} N_{3} N_{4}+N_{2} N_{3} N_{4}}$.

The following lemma computes and compares the finite sample power of a two-sided $t$-test for the hypothesis $H_{0}: \beta_{1}=0$ against $H_{1}: \beta_{1} \neq 0$ based on the short and the long regression model. We show that because the variance of $\hat{\beta}_{1}$ is larger than the variance of $\hat{\beta}_{1}^{s}$, the $t$-test based on the short model exhibits higher finite sample power than the $t$-test based on the long model. ${ }^{29}$

Let $\hat{t}^{s}=\hat{\beta}_{1}^{s} / S E\left(\hat{\beta}_{1}^{s}\right)$ and $\hat{t}=\hat{\beta}_{1} / S E\left(\hat{\beta}_{1}\right)$, let $P_{\beta_{1}}$ denote probabilities under the assumption that $\beta_{1}$ is the true coefficient and let $c_{1-\alpha / 2} \equiv \Phi^{-1}(1-\alpha / 2)$, where $\Phi^{-1}$ is the quantile function of the standard normal distribution and $\alpha \in(0,0.5)$ is the nominal significance level.

Lemma 1. Suppose that the assumptions stated in the text hold and that $\beta_{12}=0$. Then:

(i) The finite sample power of the t-tests based on the short and the long model is

$$
P_{\beta_{1}}\left(|\hat{t}|>c_{1-\alpha / 2} \mid T_{1}, T_{2}\right)=\Phi\left(\frac{\beta_{1}}{S E\left(\hat{\beta}_{1}\right)}-c_{1-\alpha / 2}\right)+1-\Phi\left(\frac{\beta_{1}}{S E\left(\hat{\beta}_{1}\right)}+c_{1-\alpha / 2}\right),
$$

and

$$
P_{\beta_{1}}\left(\left|\hat{t}^{s}\right|>c_{1-\alpha / 2} \mid T_{1}, T_{2}\right)=\Phi\left(\frac{\beta_{1}}{S E\left(\hat{\beta}_{1}^{s}\right)}-c_{1-\alpha / 2}\right)+1-\Phi\left(\frac{\beta_{1}}{S E\left(\hat{\beta}_{1}^{s}\right)}+c_{1-\alpha / 2}\right) .
$$

(ii) The t-test based on the short model is more powerful than the $t$-test based on the long model:

$$
P_{\beta_{1}}\left(\left|\hat{t}^{s}\right|>c_{1-\alpha / 2} \mid \boldsymbol{T}_{1}, \boldsymbol{T}_{2}\right) \geq P_{\beta_{1}}\left(|\hat{t}|>c_{1-\alpha / 2} \mid \boldsymbol{T}_{1}, \boldsymbol{T}_{2}\right) .
$$

${ }^{29} \mathrm{To}$ see this, note that

$$
\operatorname{Var}\left(\hat{\beta}_{1}\right)-\operatorname{Var}\left(\hat{\beta}_{1}^{s}\right)=\sigma^{2} \frac{N_{3} N_{4}\left(N_{1}+N_{2}\right)^{2}}{N_{1} N_{2}\left(N_{1} N_{2} N_{3}+N_{1} N_{2} N_{4}+N_{1} N_{3} N_{4}+N_{2} N_{3} N_{4}\right)} \geq 0 .
$$


Proof. Part (i): Under the assumptions in the statement of the lemma,

$$
\frac{\hat{\beta}_{1}-\beta_{1}}{S E\left(\hat{\beta}_{1}\right)} \mid \boldsymbol{T}_{1}, \boldsymbol{T}_{2} \sim N(0,1) .
$$

It follows that, for $z \in \mathbb{R}$,

$$
\begin{aligned}
P_{\beta_{1}}\left(\hat{t}>z \mid T_{1}, T_{2}\right) & =P_{\beta_{1}}\left(\frac{\hat{\beta}_{1}}{S E\left(\hat{\beta}_{1}\right)}>z \mid \boldsymbol{T}_{1}, \boldsymbol{T}_{2}\right) \\
& =P_{\beta_{1}}\left(\frac{\hat{\beta}_{1}-\beta_{1}}{S E\left(\hat{\beta}_{1}\right)}>z-\frac{\beta_{1}}{S E\left(\hat{\beta}_{1}\right)} \mid \boldsymbol{T}_{1}, \boldsymbol{T}_{2}\right) \\
& =\Phi\left(\frac{\beta_{1}}{S E\left(\hat{\beta}_{1}\right)}-z\right) .
\end{aligned}
$$

Thus, the power of a two-sided test is

$$
\begin{aligned}
P_{\beta_{1}}\left(|\hat{t}|>c_{1-\alpha / 2} \mid \boldsymbol{T}_{1}, \boldsymbol{T}_{2}\right) & =P_{\beta_{1}}\left(\hat{t}>c_{1-\alpha / 2} \mid \boldsymbol{T}_{1}, \boldsymbol{T}_{2}\right)+P_{\beta_{1}}\left(\hat{t}<-c_{1-\alpha / 2} \mid \boldsymbol{T}_{1}, \boldsymbol{T}_{2}\right) \\
& =\Phi\left(\frac{\beta_{1}}{S E\left(\hat{\beta}_{1}\right)}-c_{1-\alpha / 2}\right)+1-\Phi\left(\frac{\beta_{1}}{S E\left(\hat{\beta}_{1}\right)}+c_{1-\alpha / 2}\right) .
\end{aligned}
$$

Similarly, one can show that

$$
P_{\beta_{1}}\left(\left|\hat{t}^{s}\right|>c_{1-\alpha / 2} \mid T_{1}, T_{2}\right)=\Phi\left(\frac{\beta_{1}}{S E\left(\hat{\beta}_{1}^{s}\right)}-c_{1-\alpha / 2}\right)+1-\Phi\left(\frac{\beta_{1}}{S E\left(\hat{\beta}_{1}^{s}\right)}+c_{1-\alpha / 2}\right) .
$$

Part (ii): To establish the result, we show that the power is decreasing in the standard error. Using the same arguments as in Part (i), it follows that the power of a $t$-test based on an estimator $\tilde{\beta}_{1}$ which satisfies

$$
\tilde{t} \equiv \frac{\tilde{\beta}_{1}-\beta_{1}}{\operatorname{SE}(\tilde{\beta})} \mid \boldsymbol{T}_{1}, \boldsymbol{T}_{2} \sim N(0,1)
$$

is given by

$$
P_{\beta_{1}}\left(|\tilde{t}|>c_{1-\alpha / 2} \mid \boldsymbol{T}_{1}, \boldsymbol{T}_{2}\right)=\Phi\left(\frac{\beta_{1}}{\operatorname{SE}(\tilde{\beta})}-c_{1-\alpha / 2}\right)+1-\Phi\left(\frac{\beta_{1}}{\operatorname{SE}(\tilde{\beta})}+c_{1-\alpha / 2}\right) .
$$


Consider ${ }^{30}$

$$
\begin{aligned}
\frac{\partial P_{\beta_{1}}\left(|\tilde{t}|>c_{1-\alpha / 2} \mid \boldsymbol{T}_{1}, \boldsymbol{T}_{2}\right)}{\partial S E(\tilde{\beta})} & =\phi\left(\frac{\beta_{1}}{\operatorname{SE}(\tilde{\beta})}-c_{1-\alpha / 2}\right) \frac{-\beta_{1}}{\operatorname{SE}(\tilde{\beta})^{2}}-\phi\left(\frac{\beta_{1}}{S E(\tilde{\beta})}+c_{1-\alpha / 2}\right) \frac{-\beta_{1}}{S E(\tilde{\beta})^{2}} \\
& =\frac{\beta_{1}}{S E(\tilde{\beta})^{2}}\left[\phi\left(\frac{\beta_{1}}{S E(\tilde{\beta})}+c_{1-\alpha / 2}\right)-\phi\left(\frac{\beta_{1}}{S E(\tilde{\beta})}-c_{1-\alpha / 2}\right)\right] \leq 0,
\end{aligned}
$$

which follows from the shape of the normal distribution.

\section{A.3.2 Power gains and the size of the interaction cell}

Here we discuss how the power gains of the $t$-test based on the short model are related to the size of the interaction cell. Recall that in a $2 \times 2$ factorial design, the variance of the estimate of $\beta_{1}$ is given by

$\operatorname{Var}\left(\hat{\beta}_{1}\right)=\sigma^{2} \frac{N_{1}+N_{2}}{N_{1} N_{2}} \quad$ and $\quad \operatorname{Var}\left(\hat{\beta}_{1}^{s}\right)=\sigma^{2} \frac{N_{1} N_{3}+N_{1} N_{4}+N_{2} N_{3}+N_{2} N_{4}}{N_{1} N_{2} N_{3}+N_{1} N_{2} N_{4}+N_{1} N_{3} N_{4}+N_{2} N_{3} N_{4}}$.

Moreover, as shown in Lemma 1, the power of the $t$-test is decreasing in the variance of the estimator.

To illustrate, we simplify the problem by assuming that $N_{1}=N_{2}=N_{3}$, and hence that the researcher simply has to determine the relative size of $N_{4}$. Let $\alpha$ be such that $N_{4}=\alpha N$. Thus, $N_{1}=N_{2}=N_{3}=\frac{1}{3}(1-\alpha) N$. Then:

$$
\operatorname{Var}\left(\hat{\beta}_{1}\right) \equiv \sigma^{2} \frac{6}{(1-\alpha) N} \quad \text { and } \quad \operatorname{Var}\left(\hat{\beta}_{1}^{s}\right) \equiv \sigma^{2} \frac{6(1+2 \alpha)}{(1-\alpha) N(1+8 \alpha)}
$$

Figure A.1 shows how the variance changes for different values of $\alpha$. The more sample we allocate to the interaction cell, the higher the variance of $\hat{\beta}_{1}$ (i.e., the lower the power) of the long model. However, for the short model the relationship is non-monotonic. The lowest variance (highest power) is achieved when the sample size is allocated equally across cells (i.e., $\alpha=0.25$ ). Intuitively, given that we ignore the fact that some individuals get both treatments, at this point the size of the treatment and the control group for $T_{1}$ is the same.

\footnotetext{
${ }^{30}$ See, for example, Lemma 2 in Carneiro et al. (2017) for a similar argument.
} 
Figure A.1: $\operatorname{Var}\left(\hat{\beta}_{1}\right)$ and $\operatorname{Var}\left(\hat{\beta}_{1}^{s}\right)$ as the interaction cell becomes larger

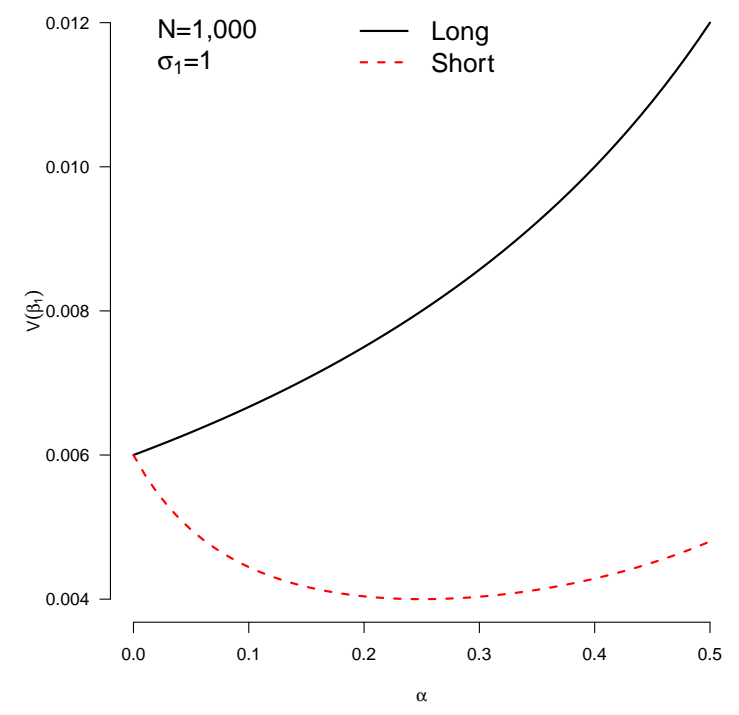

\section{A.4 Implementation details for Section 5.3}

Recall that under Assumption 1, $\beta_{12} \in\left\{b_{12}:\left|b_{12}\right| \leq C\right\} \equiv \mathcal{B}_{12}$. Hence, our problem falls into the regularized regression setting of Armstrong et al. (2019). We therefore adopt the algorithm outlined in their Section 5 to our problem. The algorithm has three steps: ${ }^{31}$

1. Obtain an estimator $\hat{\sigma}^{2}$ of $\sigma^{2}$ by taking the square root of the average of the squared residuals from estimating the long model by OLS.

2. Minimize $c v_{\alpha}\left(\frac{\left|\operatorname{Bias}\left(\hat{\beta}_{\lambda}\right)\right|}{\operatorname{SE}\left(\hat{\beta}_{\lambda}\right)}\right) \operatorname{SE}\left(\hat{\beta}_{\lambda}\right)$ with respect to $\lambda$ over $[0, \infty)$, where

$$
\begin{aligned}
\operatorname{SE}\left(\hat{\beta}_{\lambda}\right) & \equiv \sqrt{\hat{\sigma}^{2} \frac{\left\|\mathbf{T}_{1}-\mathbf{T}_{12} \pi_{\lambda}\right\|_{2}^{2}}{\left(\left(\mathbf{T}_{1}-\mathbf{T}_{12} \pi_{\lambda}\right)^{\prime} \mathbf{T}_{1}\right)^{2}}} \\
\operatorname{Bias}\left(\hat{\beta}_{\lambda}\right) & \equiv \frac{C}{\left|\pi_{\lambda}\right|} \frac{\left(\mathbf{T}_{1}-\mathbf{T}_{12} \pi_{\lambda}\right)^{\prime} \mathbf{T}_{12} \pi_{\lambda}}{\left(\mathbf{T}_{1}-\mathbf{T}_{12} \pi_{\lambda}\right)^{\prime} \mathbf{T}_{1}}
\end{aligned}
$$

and $\pi_{\lambda}$ solves $\min _{\pi}\left\|\mathbf{T}_{1}-\pi \mathbf{T}_{12}\right\|_{2}^{2}+\lambda|\pi|$. Denote the solution by $\lambda^{*}$.

\footnotetext{
${ }^{31}$ The implementation of the optimal confidence intervals with potentially heteroskedastic and nonGaussian errors mimics the common practice of applying OLS (the validity of which requires homoscedasticity) in conjunction with heteroscedasticity robust standard errors, rather than weighted least squares.
} 
3. Construct an optimal confidence interval as

$$
\hat{\beta}_{\lambda^{*}} \pm c v_{\alpha}\left(\frac{\left|\operatorname{Bias}\left(\hat{\beta}_{\lambda^{*}}\right)\right|}{\operatorname{SE}\left(\hat{\beta}_{\lambda^{*}}\right)}\right) \operatorname{SE}\left(\hat{\beta}_{\lambda^{*}}\right)
$$

where

$$
\hat{\beta}_{\lambda^{*}}=\frac{\left(\mathbf{T}_{1}-\mathbf{T}_{2} \pi_{\lambda^{*}}\right)^{\prime} \mathbf{Y}}{\left(\mathbf{T}_{1}-\mathbf{T}_{2} \pi_{\lambda^{*}}\right)^{\prime} \mathbf{T}_{1}} .
$$

In this last step, we use the residuals from the initial estimate to construct a heteroskedasticity robust version of SE $\left(\hat{\beta}_{\lambda^{*}}\right)$.

\section{A.5 Additional econometric approaches}

In this section, we discuss two additional econometric approaches.

\section{A.5.1 Model selection with a Bonferroni-style correction}

A natural approach to control size in the presence of model selection is to take a least favorable (LF) approach and to use the largest critical value across all values for the nuisance parameter (e.g., D. W. K. Andrews \& Guggenberger, 2009; Leeb \& Pötscher, 2017). However, it is well-known that this worst-case approach can exhibit poor power properties. McCloskey (2017) suggests a procedure that improves upon the LF approach, asymptotically controls size and has non-negligible power. The basic insight of this approach is that one can construct an asymptotically valid confidence interval for $\beta_{12}$. As a consequence, one can search for the largest critical value over the values of $\beta_{12}$ in the confidence interval rather than over the whole parameter space as in the LF approach. The uncertainty about the nuisance parameter $\left(\beta_{12}\right)$ and the test statistic can be accounted for using a Bonferroni-correction. Alternatively, one can adjust critical values according to the null limiting distributions that arise under drifting parameter sequences. We refer to McCloskey $(2017,2019)$ for more details as well as specific implementation details. ${ }^{32}$

\footnotetext{
${ }^{32}$ We implement the adjusted Bonferroni critical values outlined in Section 3.2 and use the algorithm "Algorithm Bonf-Adj" in the Appendix of McCloskey (2017). We employ conservative model selection and the use a tuning parameter of $0.9 \alpha$, where $\alpha$ is the nominal level of the test.
} 
Figure A.2: McCloskey (2017)'s Bonferroni-style correction controls size but does not exhibit power gains relative to the long model

(a) Size

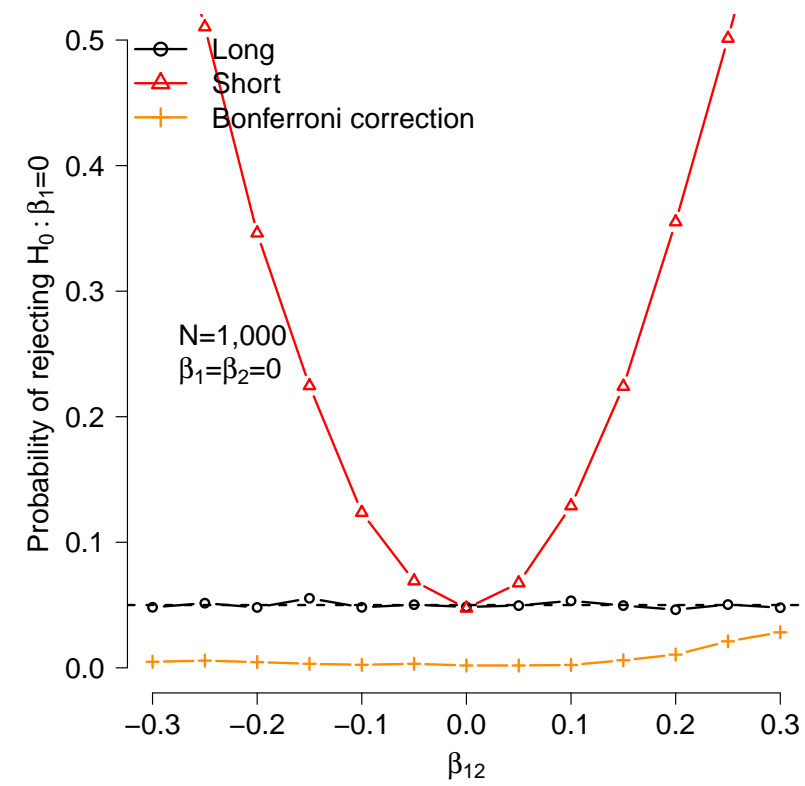

(b) Power

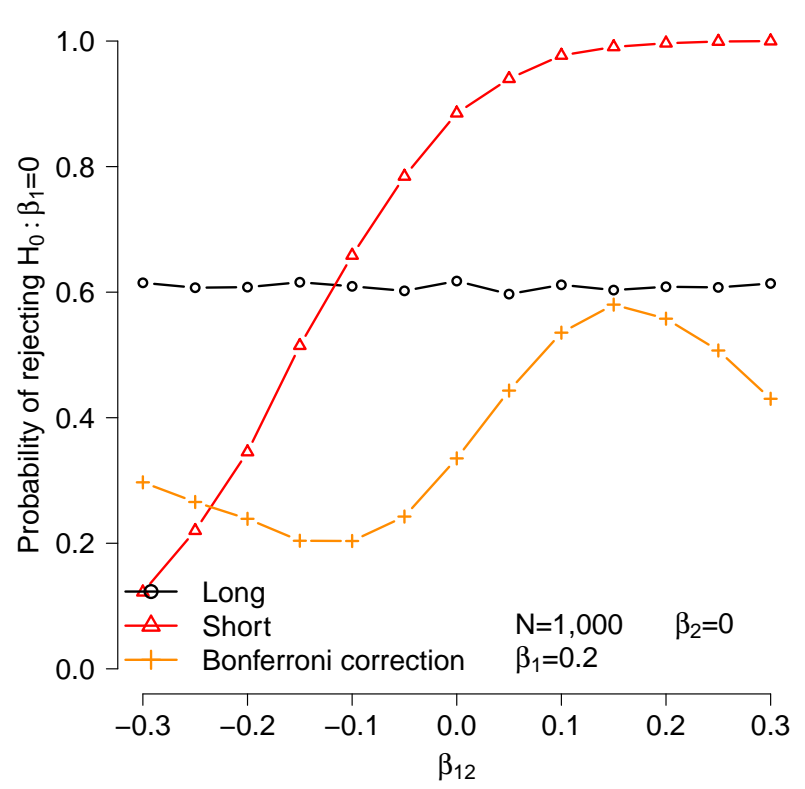

Note: Simulations are based on sample size $N$, normal iid errors, and 10,000 repetitions. The size for figures A.2a and $A .2 b$ is $\alpha=0.05$. For the model selection, the short model is estimated if one fails to reject $\beta_{12}=0$ at the $5 \%$ level.

Figure A.2 reports the results of applying McCloskey (2017)'s Bonferroni-style correction to our running example. It shows that model selection with state-of-the-art Bonferroni adjustments leads to tests that control size for all values of $\beta_{12}$. However, this method can be conservative and does not yield power gains relative to the $t$-test based on the long model, at least not over the regions of the parameter space considered here. ${ }^{33}$

\section{A.5.2 An alternative inference approach based on Assumption 1}

Here we discuss an alternative inference approach based on Assumption 1, which implies that $\beta_{12}$ lies in a compact interval,

$$
\beta_{12} \in[-C, C] \equiv\left[\beta_{12}^{l}, \beta_{12}^{u}\right] .
$$

For a given $\beta_{12} \in\left[\beta_{12}^{l}, \beta_{12}^{u}\right]$, the population regression coefficient from a regression of

\footnotetext{
${ }^{33}$ This conclusion is specific to our simulation design. Based on a different data generating process, McCloskey (2017) finds local power gains relative to the long model. However, as we discuss in Section 5.1 , the scope for improving power relative to the $t$-tests based on the long regression model is limited.
} 
$Y-\beta_{12} T_{12}$ on $X \equiv\left(1, T_{1}, T_{2}\right)^{\prime}$ is

$$
\begin{aligned}
\beta\left(\beta_{12}\right) & \equiv E\left(X X^{\prime}\right)^{-1} E\left(X\left(Y-\beta_{12} T_{12}\right)\right) \\
& =E\left(X X^{\prime}\right)^{-1} E(X Y)-\beta_{12} E\left(X X^{\prime}\right)^{-1} E\left(X T_{12}\right)
\end{aligned}
$$

Note that $E\left(X X^{\prime}\right)^{-1} E\left(X T_{12}\right) \equiv\left(\gamma_{0}, \gamma_{1}, \gamma_{2}\right)^{\prime}$ is the population regression coefficient from a regression of $T_{12}$ on $X$. Independence of $T_{1}$ and $T_{2}$ implies that $\gamma_{1}=E\left(T_{12} \mid T_{1}=\right.$ 1) $-E\left(T_{12} \mid T_{1}=0\right)$ and $\gamma_{2}=E\left(T_{12} \mid T_{2}=1\right)-E\left(T_{12} \mid T_{2}=0\right)$ both of which are positive. Consequently, the identified set for $\beta_{t}, t \in\{1,2\}$, is given by

$$
\beta_{t} \in\left\{\beta_{t}\left(\beta_{12}\right), \beta_{12} \in\left[\beta_{12}^{l}, \beta_{12}^{u}\right]\right\}=\left[\beta_{t}\left(\beta_{12}^{u}\right), \beta_{t}\left(\beta_{12}^{l}\right)\right] \equiv\left[\beta_{t}^{l}, \beta_{t}^{u}\right] .
$$

The lower bound $\beta_{t}^{l}$ can be estimated from an OLS regression of $Y-\beta_{12}^{u} T_{12}$ on X. Similarly, the upper bound $\beta_{t}^{u}$ can be obtained from an OLS regression of $Y-\beta_{12}^{l} T_{12}$ on $X$. Under standard conditions, the OLS estimators $\hat{\beta}_{t}^{l}$ and $\hat{\beta}_{t}^{u}$ are asymptotically normal and the asymptotic variances Avar $\left(\hat{\beta}_{t}^{l}\right)$ and Avar $\left(\hat{\beta}_{t}^{u}\right)$ can be estimated consistently. We can therefore apply the approach of Imbens \& Manski (2004) and Stoye (2009) to construct confidence intervals for $\beta_{t}: 34$

$$
C I_{1-\alpha}=\left[\hat{\beta}_{t}^{l}-c_{I M} \cdot \sqrt{\frac{\widehat{A v a r}\left(\hat{\beta}_{t}^{l}\right)}{N}}, \hat{\beta}_{t}^{u}+c_{I M} \cdot \sqrt{\frac{\widehat{\operatorname{Avar}}\left(\hat{\beta}_{t}^{u}\right)}{N}}\right],
$$

where the critical value $c_{I M}$ solves

$$
\Phi\left(c_{I M}+\sqrt{N} \cdot \frac{\hat{\beta}_{t}^{u}-\hat{\beta}_{t}^{l}}{\sqrt{\max \left(\widehat{\operatorname{Avar}}\left(\hat{\beta}_{t}^{l}\right), \widehat{\operatorname{Avar}}\left(\hat{\beta}_{t}^{l}\right)\right)}}\right)-\Phi\left(-c_{I M}\right)=1-\alpha .
$$

Imbens \& Manski (2004) and Stoye (2009) show that (21) is a valid confidence interval for $\beta_{t}$.

In Figure A.3, we report the rejection probabilities of a test that rejects if zero is not in the confidence interval (21). For the purpose of illustration, we assume that $C=0.1$ which implies that $\beta_{12} \in[-0.1,0.1]$. Our results suggest that imposing prior knowledge can improve power relative to the long regression model, while controlling size when

\footnotetext{
${ }^{34}$ By construction, the upper bound is always weakly larger than the lower bound. Hence Lemma 3 in Stoye (2009) justifies the procedure in Imbens \& Manski (2004).
} 
this prior knowledge is in fact correct. However, this method exhibits substantial size distortions when the prior knowledge is incorrect.

Figure A.3: Restrictions on the magnitude of $\beta_{12}$ yield power gains if they are correct but lead to incorrect inferences if they are not

(a) Size

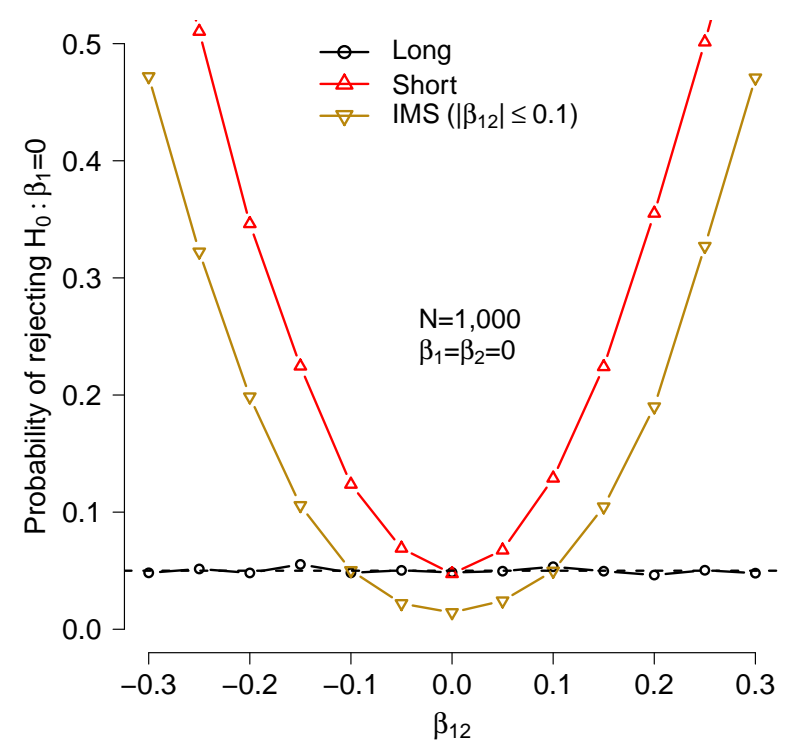

(b) Power

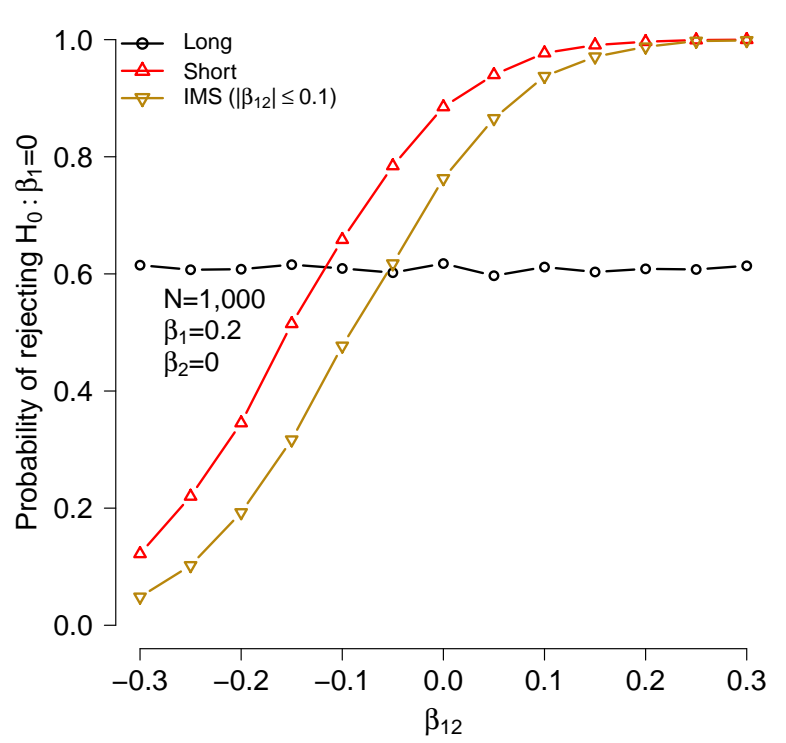

Note: Simulations are based on sample size $N$, normal iid errors, and 10,000 repetitions. The size for figures A.3a and $A .3 b$ is $\alpha=0.05$. IMS refers to Imbens \& Manski (2004) and Stoye (2009) approach for constructing valid confidence intervals under prior knowledge about the magnitude of $\beta_{12}$.

\section{A.6 Additional figures and tables}

Table A.1: Articles published in top-5 journals between 2007 and 2017

\begin{tabular}{lcccccc}
\hline & AER & ECMA & JPE & QJE & ReStud & Total \\
\hline Other & 1218 & 678 & 367 & 445 & 563 & 3271 \\
Field experiment & 43 & 9 & 14 & 45 & 13 & 124 \\
Lab experiment & 61 & 16 & 5 & 10 & 18 & 110 \\
Total & 1322 & 703 & 386 & 500 & 594 & 3505 \\
& & & & & & \\
\hline
\end{tabular}




\section{A.6.1 Ignoring the interaction}

Figure A.1: Long and short model: Size and power

(a) Size
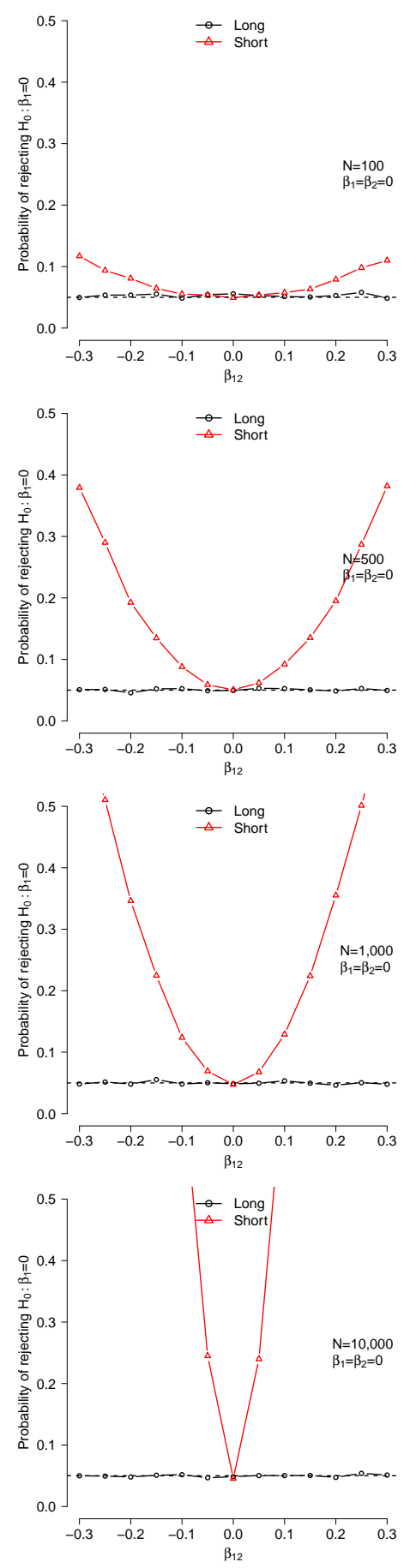

(b) Power
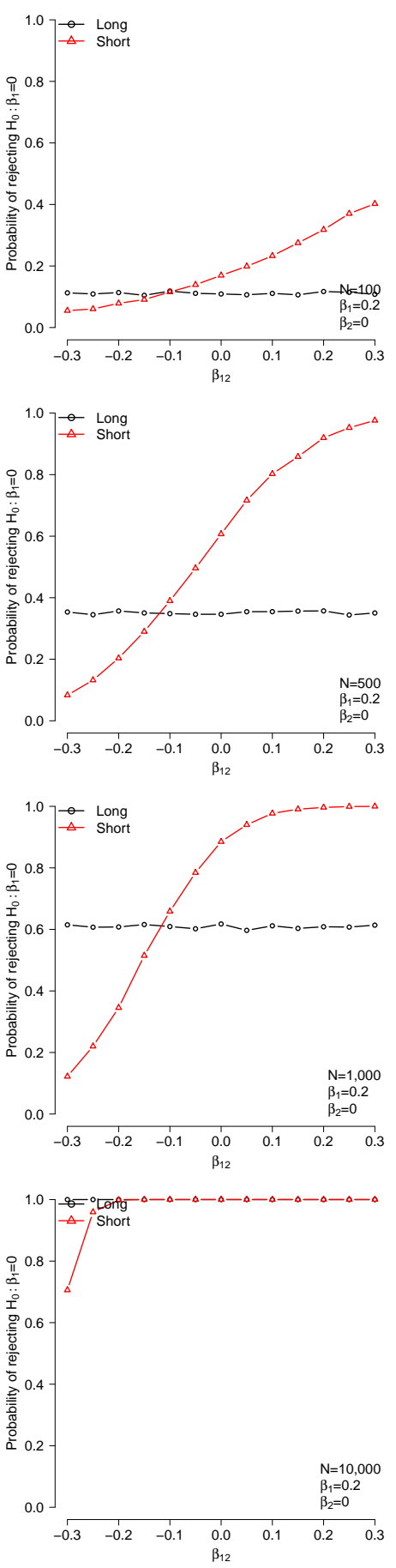

Note: Simulations are based on sample size $N$, normal iid errors, and 10,000 repetitions. The size across all figures is $\alpha=0.05$. 


\section{Figure A.2: Long and short model: Power curves}
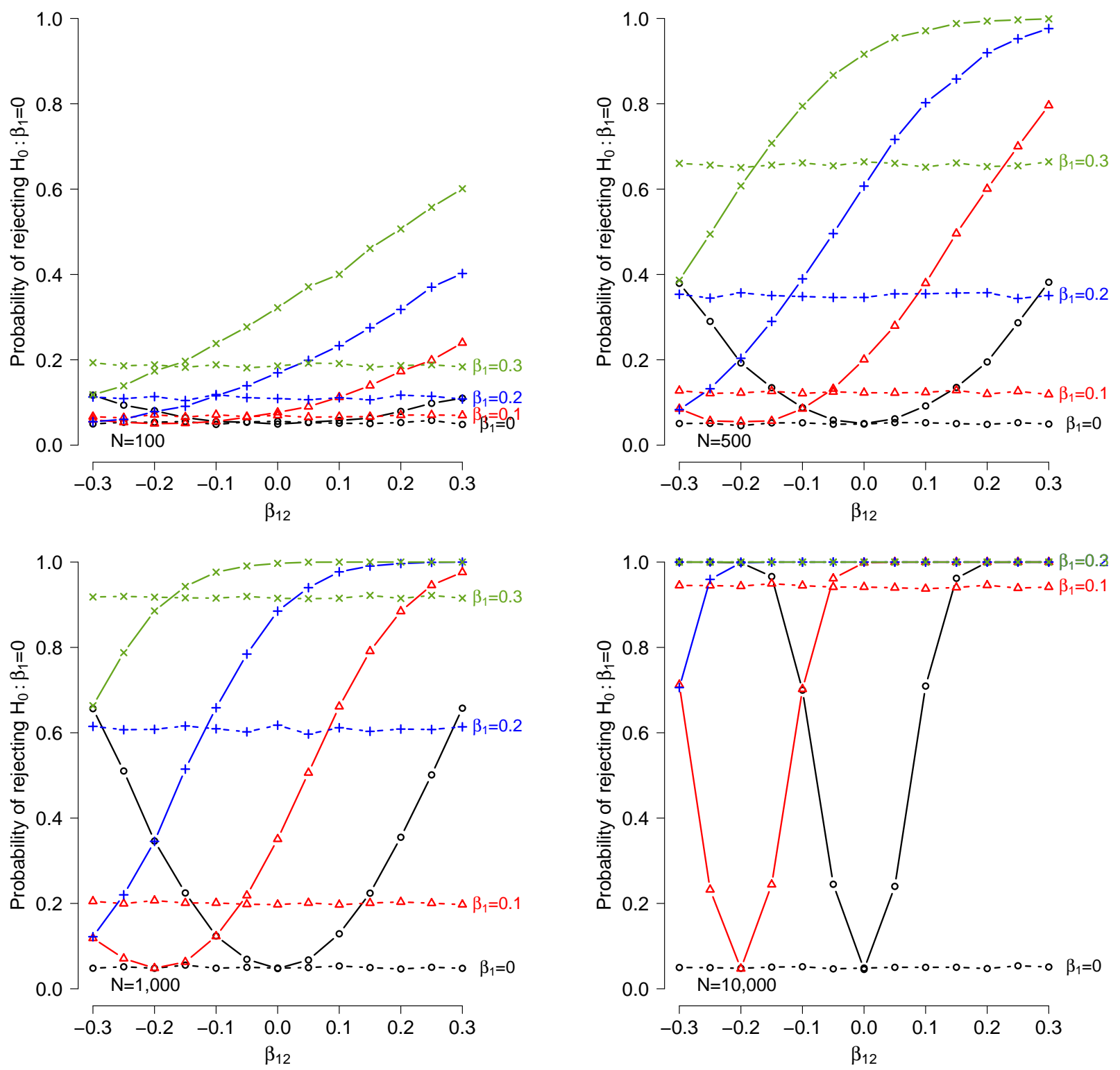

Note: Simulations are based on sample size $N$, normal iid errors, and 10,000 repetitions. The size across all figures is $\alpha=0.05$. In each figure, dashed lines show the power for the long model, while solid lines show power for the short model. 


\section{A.6.2 Model selection (pre-testing)}

Figure A.3: Model selection: Size and power

(a) Size
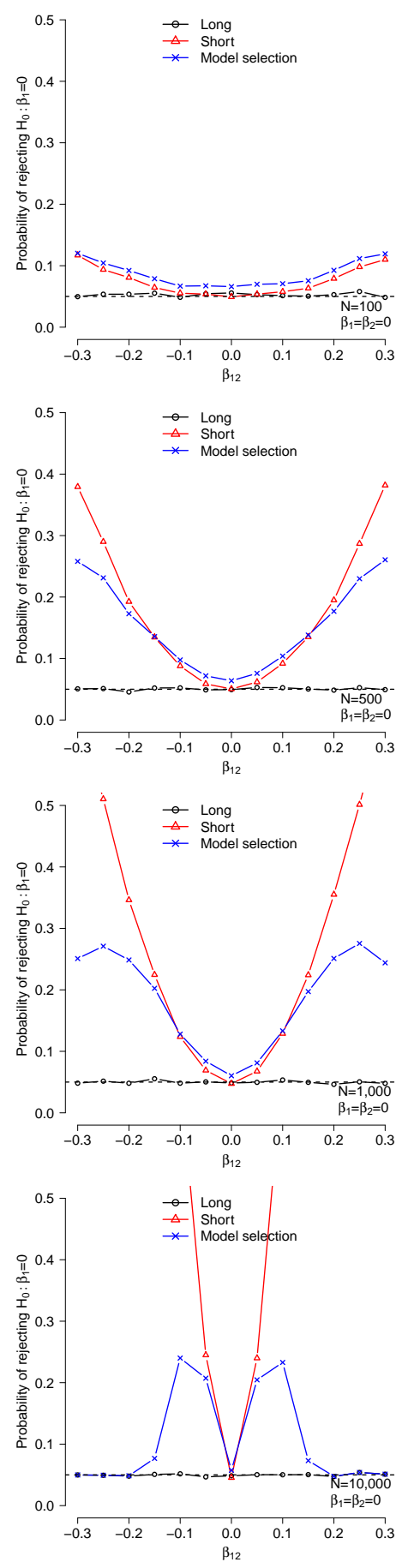

(b) Power
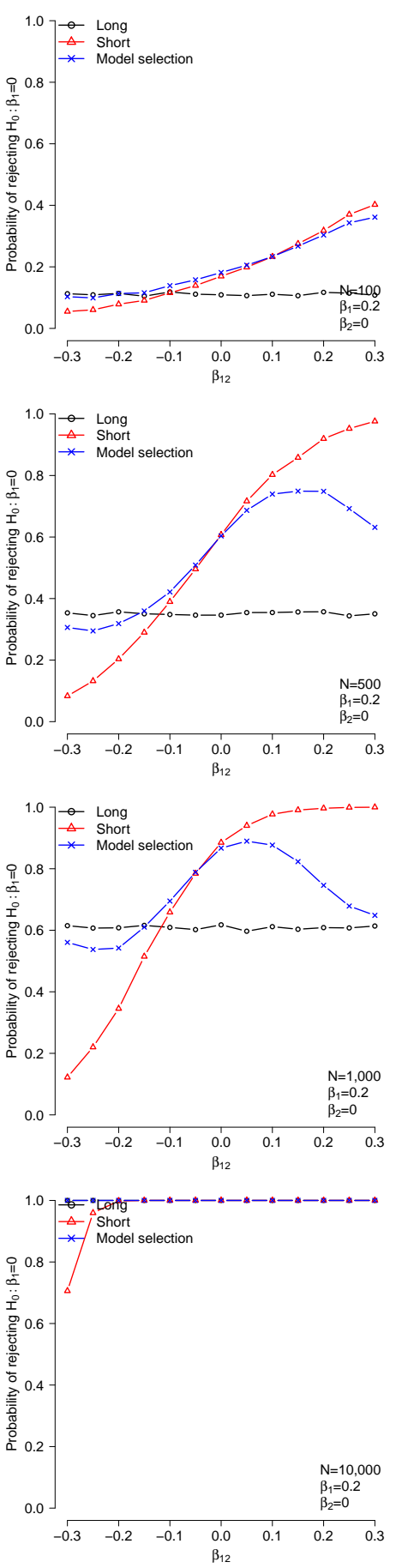

Note: Simulations are based on sample size $N$, normal iid errors, and 10,000 repetitions. The size across all figures is $\alpha=0.05$. For the model selection, the short model is estimated if one fails to reject $\beta_{12}=0$ at the $5 \%$ level. 


\section{A.6.3 Elliott et al. (2015)'s nearly optimal test}

Figure A.4: Elliott et al. (2015)'s nearly optimal test: Size and power

(a) Size
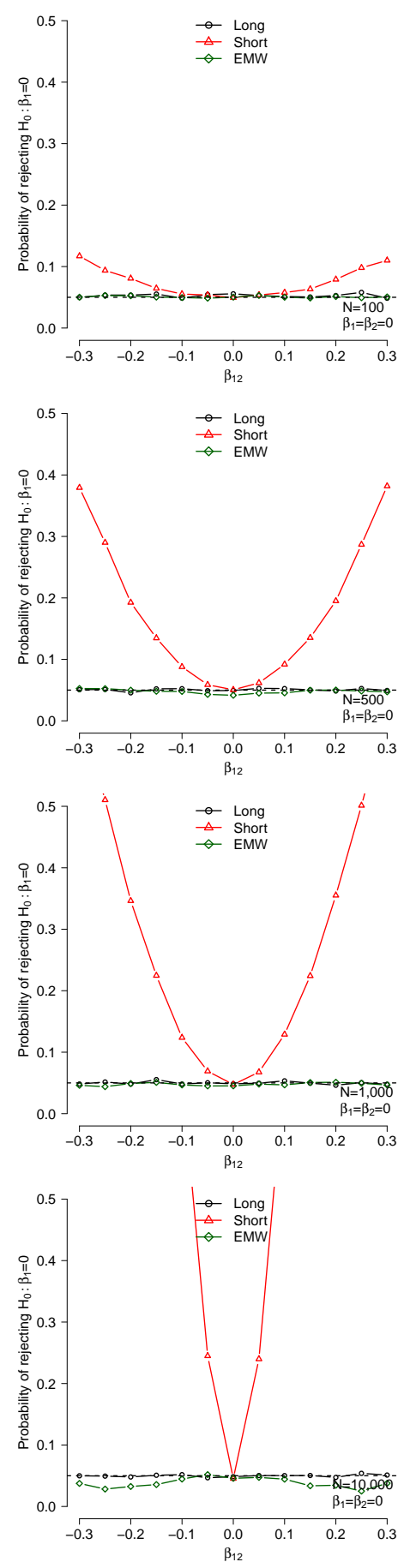

(b) Power
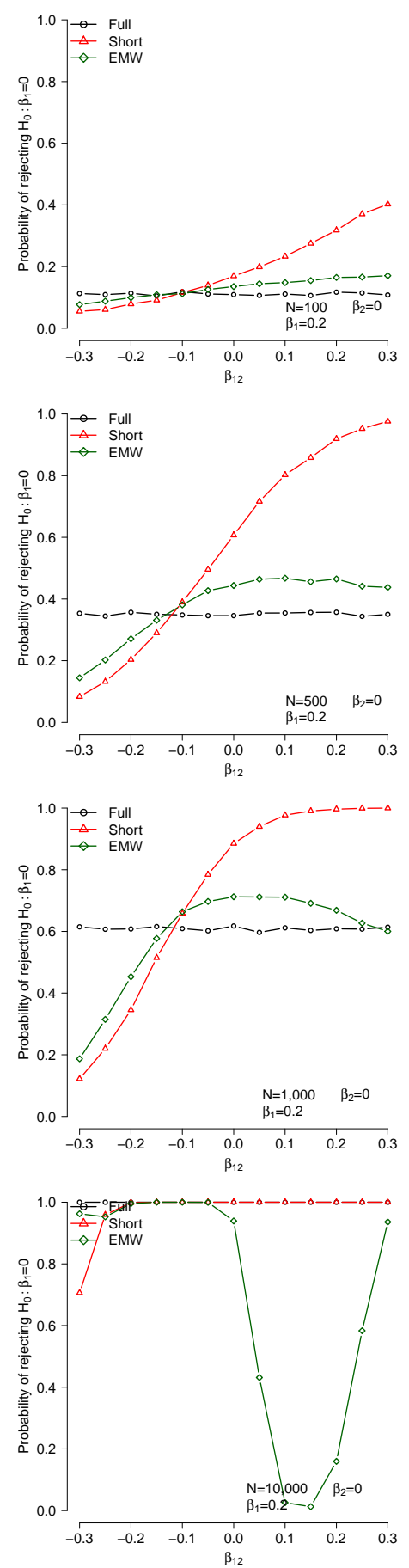

Note: Simulations are based on sample size $N$, normal iid errors, and 10,000 repetitions. The size across all figures is $\alpha=0.05$. 
Figure A.5: Long model and Elliott et al. (2015)'s nearly optimal test: Power curves
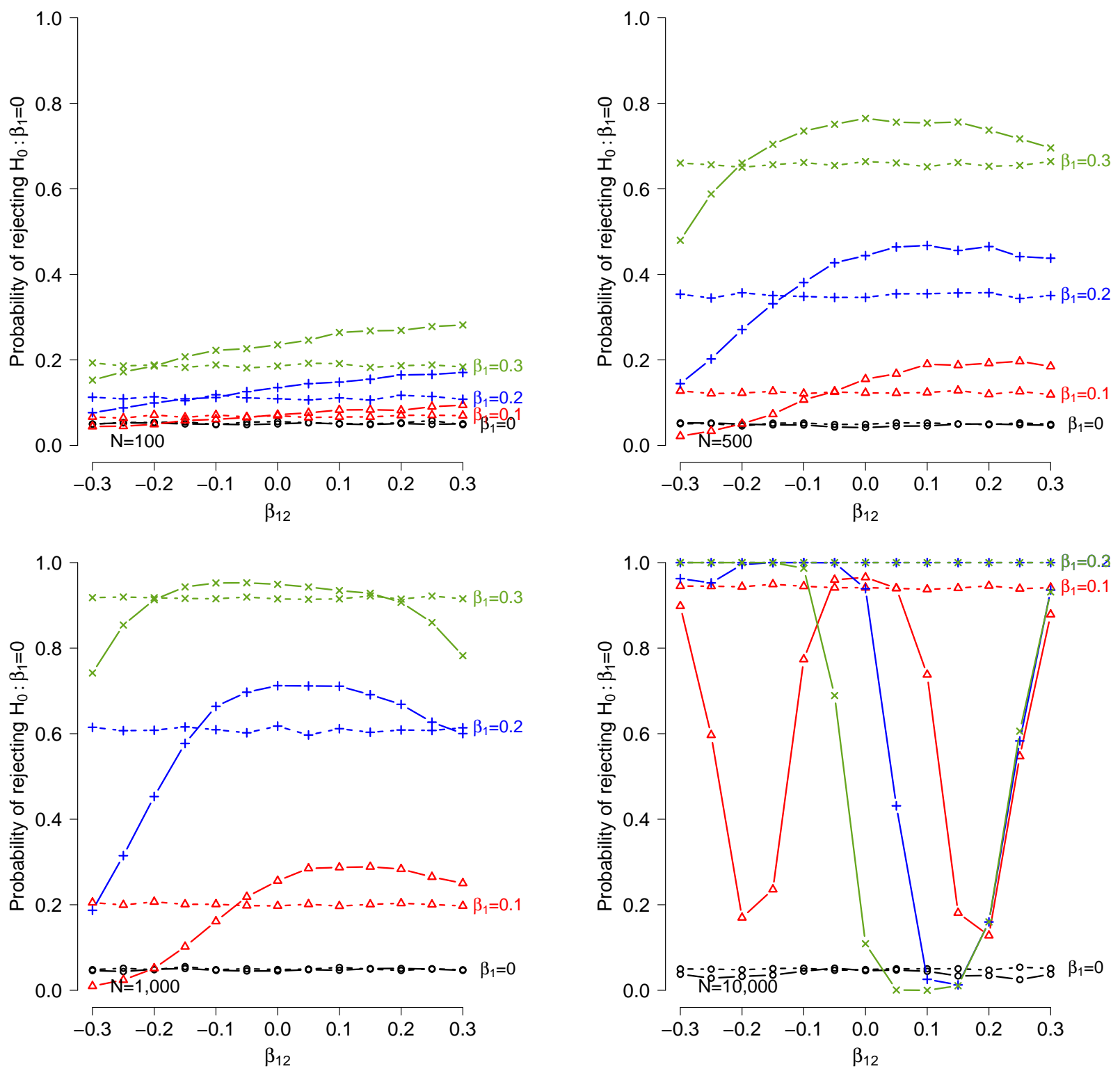

Note: Simulations are based on sample size $N$, normal iid errors, and 10,000 repetitions. The size across all figures is $\alpha=0.05$. In each figure, dashed lines show the power for the long model, while solid lines show power for Elliott et al. (2015)'s nearly optimal test. 


\section{A.6.4 Restrictions on the magnitude of $\beta_{12}$ : Armstrong et al. (2019)}

Figure A.6: Armstrong et al. (2019)'s approach: Size and power

(a) Size
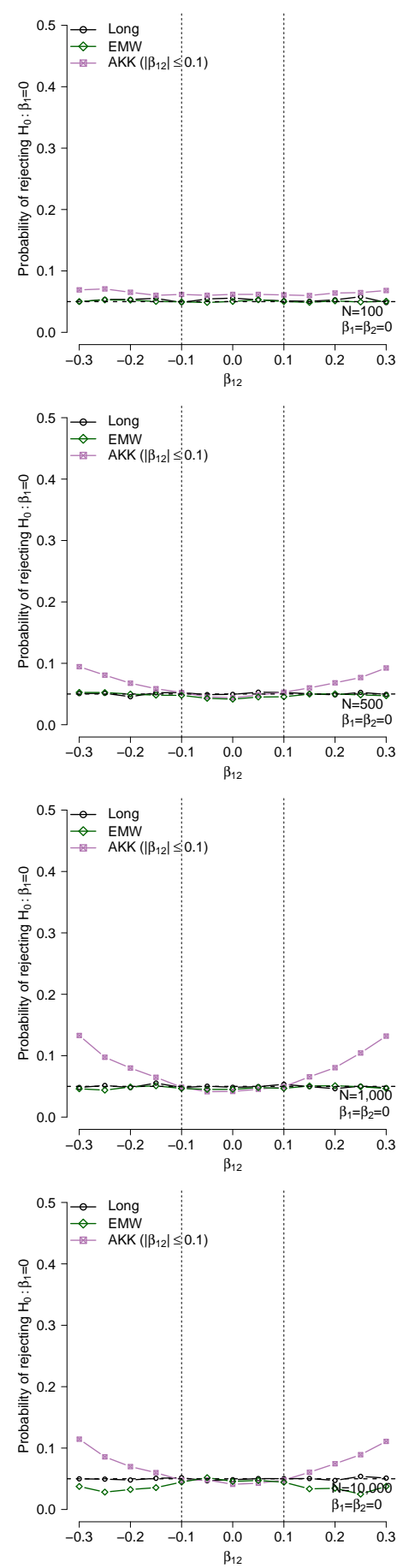

(b) Power
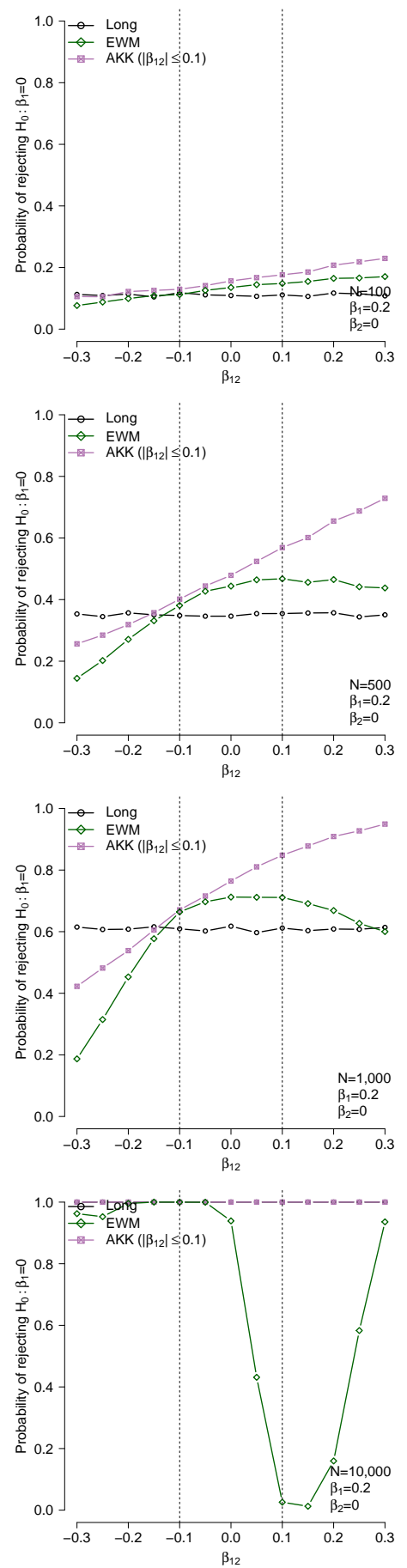

Note: Simulations are based on sample size $N$, normal iid errors, and 10,000 repetitions. The size across all figures is $\alpha=0.05$. 
Figure A.7: Long model and Armstrong et al. (2019)'s approach: Power curves
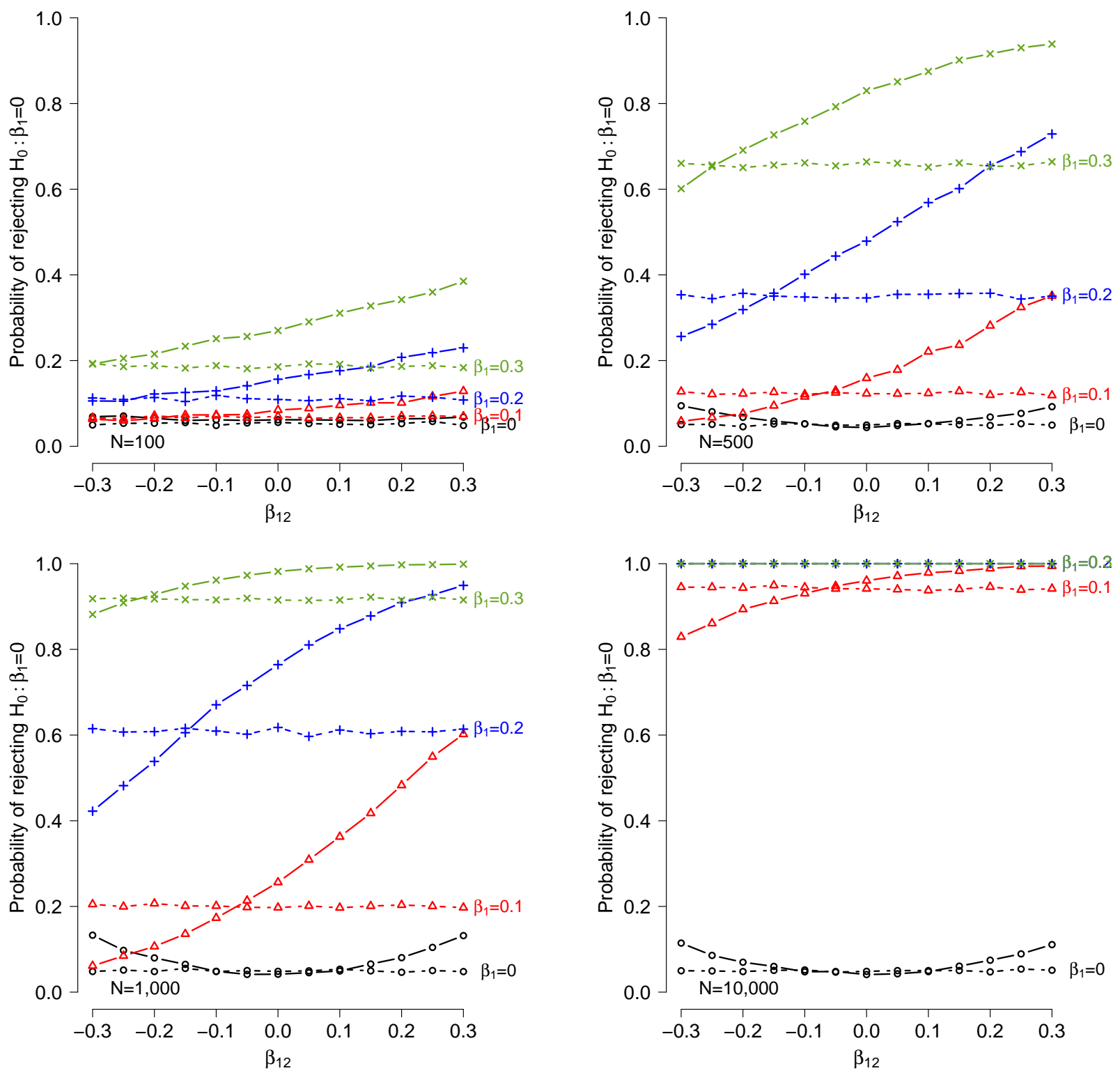

Note: Simulations are based on sample size $N$, normal iid errors, and 10,000 repetitions. The size across all figures is $\alpha=0.05$. In each figure, dashed lines show the power for the long model, while solid lines show power for Armstrong et al. (2019)'s approach based on restrictions on the magnitude of $\beta_{12}$. 


\section{A.6.5 Leaving the interaction cell empty}

Figure A.8: No factorial design: Size and power

(a) Size
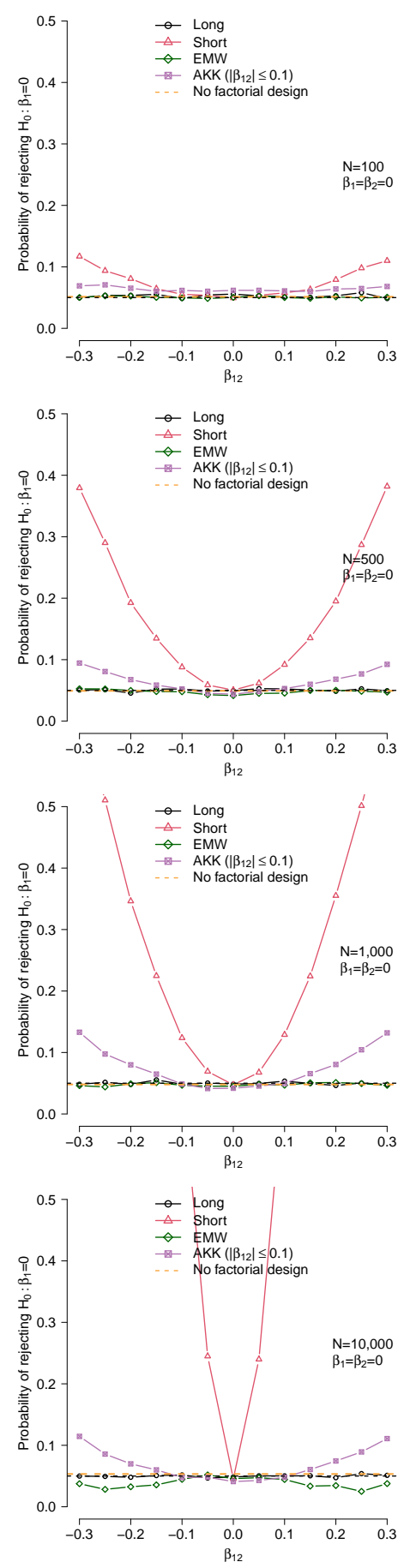

(b) Power
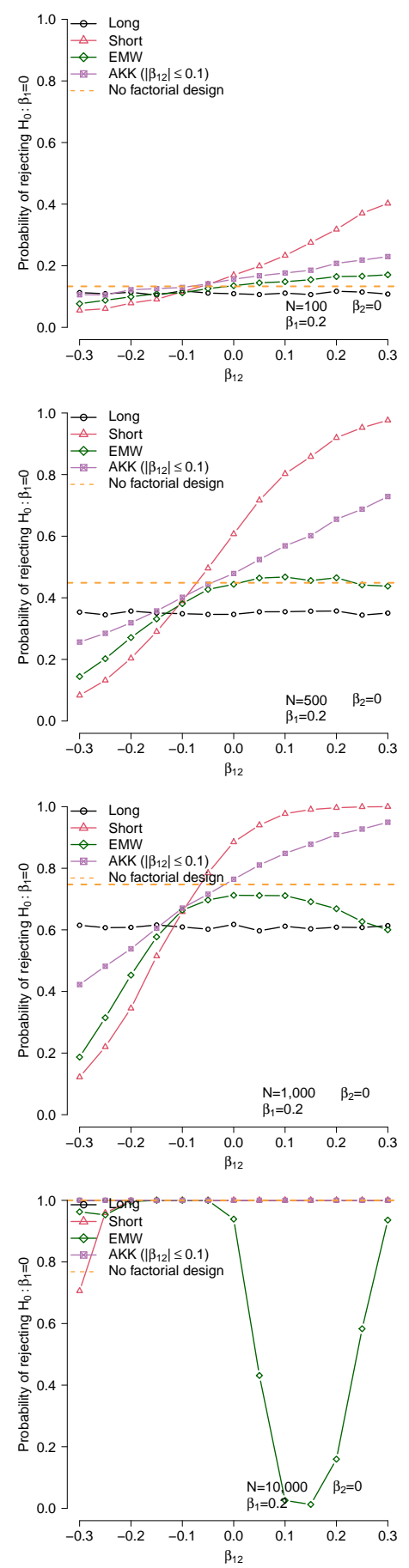

Note: Simulations are based on sample size $N$, normal iid errors, and 10,000 repetitions. $N_{T}^{*}=0.29 \mathrm{~N}$ and $N_{1}^{*}=0.42 N$. The size across all figures is $\alpha=0.05$. 